\title{
Free cooling of granular particles with rotational degrees of freedom
}

\author{
Dissertation \\ zur Erlangung des Doktorgrades \\ der Mathematisch-Naturwissenschaftlichen Fakultäten \\ der Georg-August-Universität zu Göttingen
}

vorgelegt von

Martin Huthmann

aus Memmingen

Göttingen 1999 
D7

Referentin: Prof. Dr. A. Zippelius

Korreferent: Prof. Dr. R. Kree

Tag der mündlichen Prüfung: 4. 11. 1999 


\section{Contents}

1. Introduction $\quad \mathbf{1}$

1.1. Undriven rapid granular flow . . . . . . . . . . . . . . . . . . . 3

1.2. Methods ...................... 5

1.2.1. Kinetic theory and hydrodynamic description . . . . . . . 5

1.2.2. Simulational methods . . . . . . . . . . . . . . . . 6

1.3. Questions to be answered ............... 8

$\begin{array}{ll}\text { 2. The Liouville operator } & \mathbf{1 0}\end{array}$

2.1. Classical systems . . . . . . . . . . . . . . . . . . 11

2.1.1. Smooth potentials . . . . . . . . . . . . . 11

2.1.2. Elastic hard-core interactions . . . . . . . . . . . . . 11

2.2. Inelastic collision . . . . . . . . . . . . . . . . . . . . . . . . . . . . . . 17

2.2.1. Rough spheres . . . . . . . . . . . . . . . . . 17

2.2.2. Rough needles . . . . . . . . . . . . . . . . 17

2.3. Time evolution of the distribution function . . . . . . . . . . . 18

2.4. Event-driven simulations . . . . . . . . . . . . . . . 20

2.4.1. General ideas . . . . . . . . . . . . . . . 20

2.4.2. Hard needles . . . . . . . . . . . . . . . . . . . . . . . . . . . . . . . . .

2.4.3. Hard spheres . . . . . . . . . . . . . . . . . . . . . . . . . . . . 22

2.5. Summary .............................. 22

3. Homogeneous cooling state $\quad 24$

3.1. Gaussian distribution . . . . . . . . . . . . . . . . 26

3.1.1. Results for spheres . . . . . . . . . . . . . . 27

3.1.2. Results for needles . . . . . . . . . . . . . . . . 35

3.1.3. Summary . . . . . . . . . . . . . . . . . . . . . . . . . . . . . . . . . 38

3.2. Non-Gaussian distribution . . . . . . . . . . . . . . . . . . . . . . . . . . . . . . . . . . . . . . 39

3.2.1. Smooth spheres . . . . . . . . . . . . . . . 39

3.2.2. Rough spheres. . . . . . . . . . . . . . . . . . . . . . . . . . . . . 49

3.3. Summary . . . . . . . . . . . . . . . . . 53 
4. Inhomogeneities $\mathbf{5 5}$

4.1. Needles . . . . . . . . . . . . . . . . . . . 56

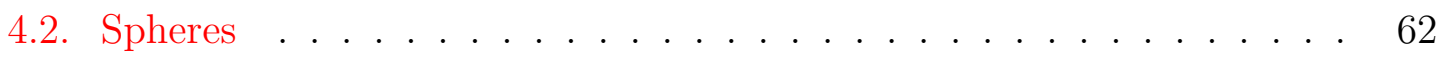

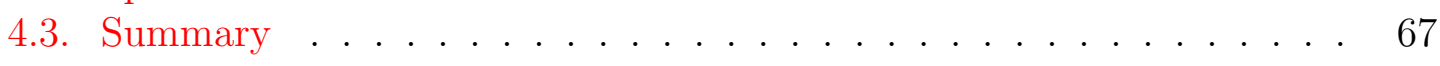

5. The Enskog Boltzmann equation $\quad \mathbf{6 8}$

5.1. The assumptions . . . . . . . . . . . . . . . 68

5.2. Equivalence of description . . . . . . . . . . . . . . . . 69

6. Conclusions and outlook $\quad 71$

A. Appendix $\mathbf{7 4}$

A.1. Calculations for spheres . . . . . . . . . . . . . . 74

A.1.1. An example . . . . . . . . . . . . . . . . . 74

A.1.2. General collision integral . . . . . . . . . . . . . . . 76

A.2. Calculations for needles . . . . . . . . . . . . . . . . . . 77

A.3. Generalized Laguerre polynomials . . . . . . . . . . . . . . . . . 81

A.4. The time dependence of the moments $1 \ldots \ldots$. . . . . . . . 82

A.5. The time dependence of the moments $2 \ldots \ldots$. . . . . . . 84

A.6. Collision integrals with MAPLE . . . . . . . . . . . 87 


\section{Introduction}

Granular materials are large conglomerates of discrete macroscopic particles and therefore obey purely classical behavior. This seems to make the description of granular matter trivial but granular materials behave differently from any of the other standard and familiar forms of matter: solids, liquids, or gases. For example it is possible to stand on a big heap of sand, which means that sand can support a certain load and can indeed behave like a solid object even though it consists of single grains. But the same sand can flow through a hopper and behave like a fluid. Fluidization is a further extraordinary phenomenon: At a certain frequency and amplitude vibrating sand can change its behavior from solid-like to fluid-like.

Two main features contribute to the various properties of granular materials: 1) Interactions between particles are strongly dissipative (static friction, and, for moving grains, the inelastic nature of their collisions) and 2) thermal fluctuations can be disregarded.

As far as statics is concerned, granular matter can be viewed as a classical system of particles at zero temperature which is confined to one of many possible meta-stable states. For example the sand pile can behave like a solid; the material remains at rest due to static friction forces, even though gravitational forces create macroscopic stress on its surface. In ordinary gases temperature allows the system to explore phase space, but in granular matter the relevant energy scale is the potential energy which is for ordinary sand $10^{12}$ times larger than $k_{B} T$ at room temperature [JaNa96]. So temperature can be neglected and unless perturbed by external disturbances each meta-stable configuration will last indefinitely.

Flow of grains occurs when the system is driven by external forces. In fig. 1.1 we see an avalanche at the surface of a pile of mustard seeds. It shows that this flow obviously need not be that of an ordinary fluid: Simultaneously fluid-like behavior in a boundary layer at the pile's surface and a solid-like phase in the bulk separated only by a few particle diameters can be observed. Roughly two regimes of grain flow are distinguished: 1) In the quasi static or slow flow regime all particles are in contact with each other permanently. Interactions are then dominated by friction forces and steric hindrance, and the particles slide or role on each other. 2) When driven by strong external agents (gravity, shear, vibrating plates), granular matter can be viewed as a complex fluid of hard particles that move ballistically and suffer instantaneous and inelastic collisions. In this rapid flow or grain inertia regime the dynamics 


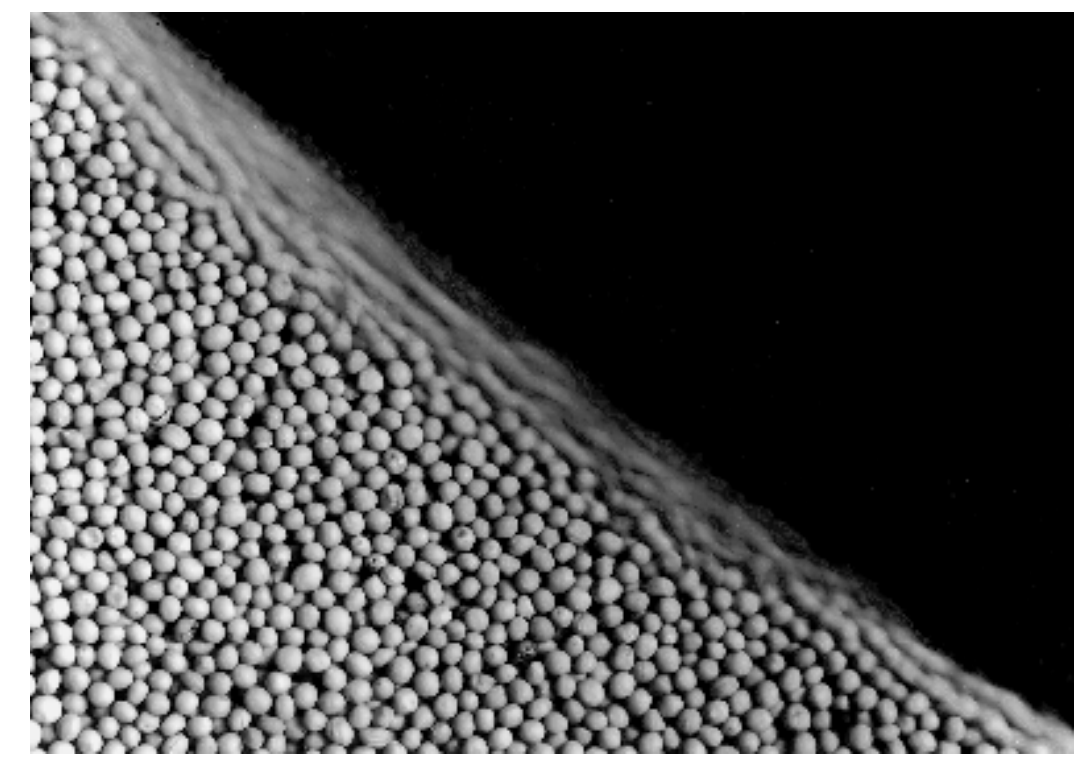

Figure 1.1.: An avalanche at the surface of a pile of mustard seeds. Note that the flow occurs in a thin layer at the surface and that the seeds deeper within the pile do not participate in the motion. The picture is taken from [ChGr99].

is dominated by inter-grain collisions with peculiar effects due to inelasticity. Fig. 1.1 also illustrates that the two described regimes are not well-defined physical phases and there exists a crossover regime, which might not easily be defined.

In general, fluid behavior only sets in above a certain threshold excitation level. After the energy input is stopped, the inelastic collisions bring the granular medium to rest almost instantly. The kinetic energy stored in translational or rotational movement is then lost rapidly to internal degrees of freedom of the granular particles. Here collective effects play an important role: While a single steel ball dropped on a plate may bounce for a while, a filled sack with the same balls will stop short any movement. The huge number of rapid inelastic collisions of neighboring grains are responsible for this effect. Gravity intensifies this by concentrating particles at the ground leading to even higher collision rates and therefore loss of energy.

In this introduction we will not give a complete overview of the huge variety of phenomena in granular matter, which can be found for example in [JaNa96, HeHo98, Ge99, Ka99], but focus on models and methods describing rapid granular flow. Generally three levels of descriptions are possible [Gi96]: On a microscopic level each particle has its own internal degrees of freedom, which can take over parts of the kinetic energy and are therefore responsible for the dissipative mechanism. A mesoscopic level where each particle is considered as a classical particle interacting with the other particles in a way that energy is not conserved. Then interaction laws 
are commonly introduced in a more phenomenological way and on that level of description granular matter can be considered as, in a thermodynamic sense, an open system with an energy sink, which is created by the dissipative interaction [NoEr99]. On a more macroscopic level one is concerned with hydrodynamic quantities like granular temperature, flow, or density, which might evolve in a particular way due to the dissipative interaction. This makes it obvious that interesting phenomena in granular matter take place in systems far away from equilibrium, indicating that granular materials, despite of the huge technical interest, are per se a challenging field to test established ideas, or to develop new techniques in the field of non-equilibrium statistical mechanics.

In the following we give a short overview of models and methods used to describe undriven rapid granular flow on a mesoscopic and macroscopic level.

\subsection{Undriven rapid granular flow}

Although the variety of phenomena in granular matter is intriguing and the interest of many theoretical physicist in granular matter is certainly motivated by a lot of beautiful experiments, we are still far from a complete theoretical description and have to restrict ourselves to simplified situations and models. In this study we focus on the grain inertia regime or rapid granular flow. In addition, we restrict ourselves to undriven systems. Driven systems are still an open problem and there is a great lack of real understanding, whereas for example the system of freely cooling ${ }^{1}$, smooth spheres, described below, has been widely investigated by several groups by means of kinetic theory and computer simulations [GoZa93, GoZa93b, Na93, NaYo96, NoEr97, NoBr98, BrMo96, BrMo98, Deba97]. This system is by now established as a kind of reference system and the hope is that it gathers all generic properties of undriven granular gases despite of its simplifying assumptions.

\section{Smooth spheres}

In this model one considers identical, spherical particles whose collision properties are described by a single normal coefficient of restitution $e_{n}$. If $\boldsymbol{V}$ is the relative velocity of two spheres, then in an elastic collision the component of $\boldsymbol{V}$ parallel to the unit vector $\hat{\boldsymbol{n}}$ pointing from one sphere to the other is completely reversed $\boldsymbol{V}^{\prime} \hat{\boldsymbol{n}}=-\boldsymbol{V} \boldsymbol{n}$ (primed quantities denote the values after collision), and to model inelasticity this reflection is only partial and determined by $e_{n} \in[0,1]$ by $\boldsymbol{V}^{\prime} \hat{\boldsymbol{n}}=-e_{n} \boldsymbol{V} \hat{\boldsymbol{n}}$.

Usually, a system prepared in a homogeneous, equilibrium state of a classical hard-sphere gas is considered. After dissipation has been switched on, due to inelastic collisions, energy is lost and the system cools down to a state where all particles are

\footnotetext{
${ }^{1}$ The term 'cooling' describes the slowing down of the (irregular) movement of the particles coming to rest in the long-time limit.
} 
at rest. The interesting feature of this systems is that it is unstable against spatial density fluctuations, which grow slowly to macroscopic clusters of particles. The reason for this so-called cluster instability has been traced back to noise reduction [BrEr99].

Cooling of smooth spheres proceeds in three stages: The particles, initially prepared in a spatial homogeneous state, remain for short times in the homogeneous cooling state (HCS), where the dynamics can be described by a slowly decreasing average kinetic energy or granular temperature ${ }^{2}$. This temperature $T$ then follows Haff's law $T \propto t^{-2}, t$ being time [Ha83]. The velocity distribution function in the HCS is no longer Gaussian [GoSh95], but only a few collisions per particle are necessary to reach a state where the shape of the distribution function is fixed and the dynamics is described by the granular temperature only [HuOr99]. With time, due to the inelastic collision, the particles move more and more parallel and inhomogeneities show up first in the flow field, giving rise to vortex patterns. The hydrodynamic analysis shows that fluctuations in the flow field are only relatively unstable, i.e. only if one measures the flow in units of the actual average velocity growing correlations are visible. In other words, the correlations in the flow field decay slower than the average energy, so that the correlations in the flow field can survive. Only later in a third stage clusters emerge due to coupling of the vorticity field to the density field [NoBr98], and the cluster instability is unstable in an absolute sense. In this late stage the kinetic energy decays much slower than in the HCS. Most of the energy is stored in the energy of the macroscopic flow field, which decays only diffusively [BrEr98].

\section{Advanced collision rules}

Further concepts of modeling undriven granular flow include more sophisticated collision rules or rotational degrees of freedom. In molecular-dynamic simulations phenomenological equations of motion are formulated which include viscous and friction forces. We will describe this techniques and quote literature in section 1.2.2. A velocity-dependent coefficient of restitution has been derived by means of a viscoelastic theory based on Hertz' law of contact [BrSp96]. A more microscopic model is proposed in [GiZi96, AsGi98], where kinetic energy can be transfered in vibrations of one-dimensional rods, an effective mechanism of energy loss derived from a Hamiltonian model. Collision properties of two-dimensional elastic disks are discussed in [GeZi99] and found to be inelastic, in general, with a finite fraction of translational energy being transfered to elastic vibrations.

Theories including rotational degrees of freedom are modeled by a constant tan-

\footnotetext{
${ }^{2}$ In this case granular temperature denotes the average energy per particle and degree of freedom, so that in this sense it can be seen analogous to the term temperature in equilibrium statistical mechanics. But in contrast to an equilibrium situation this granular temperature is not a fixed parameter and can change in time.
} 
gential coefficient of restitution as proposed and discussed in chapter 3, where we also give hints to studies in this field. More realistic models of rough spheres contain Coulomb friction, a model, whose collision properties can be confirmed fairly well by experiments [FoLo94].

\section{Non-spherical objects}

Most analytical and numerical studies of kinetic phenomena concentrate on spherical objects so far. Exceptions are computer simulations of polygonal particles [Wa88, HoSh87] and cellular automata models [BaBe90]. The question then arises which of the results are specific to spherical objects and which are generic for inelastically colliding particles. A single collision of two arbitrarily shaped, but convex objects is quite difficult to describe analytically [Br96], set aside the problem of an ensemble of colliding grains. In this work we have chosen the simplest non-spherical objects, needles, which allow for an analytical, though approximate solution and large scale simulations [HuAs99].

\subsection{Methods}

So far we have confined our interest to undriven rapid granular flow, and we now will give a short overview of the different methods used in this field.

\subsubsection{Kinetic theory and hydrodynamic description}

Kinetic theory in the context of rapid flow in granular media is almost exclusively connected with the hard-sphere model, which uses the idea of freely moving particles which suffer instantaneous inelastic binary collisions.

\section{Classical gases}

Since the hard-sphere model has been a very useful reference system for our understanding of classical liquids [Ha86], many results and techniques are available and not surprisingly the model has become very popular also in the context of granular media.

As far as static correlations are concerned, an analytical expression for the pair correlation is available [Th63, We63, VeLe82]. This provides a good first approximation for particles interacting via smooth potential functions. The hard-sphere model is even more important for the dynamics, because it allows for approximate analytical solutions based on the Boltzmann equation and its generalization by Enskog to account for a finite particle diameter and pair correlations at contact [LePe69, KoLe64, FuMa75, BeEr79]. The model has the additional advantage that it is particularly well-suited for numerical simulations [AlGa70] and in fact many of 
the important phenomena of dense liquids were observed first in simulations of hard spheres. Examples are the discovery of long-time tails [ErWo81] and two-dimensional solids [AlWa62].

\section{Granular gases}

Focusing on the rapid flow regime, where kinetic theory should apply, generalized Boltzmann and Enskog equations have been formulated and pioneering work can be found in [Ha83, JeRi85, LuSa87, Lu91]. The success of the Enskog Boltzmann equation to describe classical fluids is based on the linearization of the collision operator around local equilibrium. The resulting linear hermitian operator can then be treated by standard methods of functional analysis [Gr58, Wa58, ChCo60]. For inelastic systems no analog of the local equilibrium distribution is known.

In many studies, including the present one, a homogeneity assumption is made (HCS), which is known to be unstable for dense and large enough system and long times as described in the previous section [GoZa93]. Hence the analysis is restricted to small and moderate densities. The velocity distribution function in the homogeneous cooling state is no more Maxwellian and deviations can be investigated by an expansion in Sonine polynomials around the Gaussian state [GoSh95, NoEr98b, BrPo99, HuOr99].

\section{Hydrodynamic description}

A Chapman-Enskog-like expansion around the HCS or Grad's moment method allow for calculation of transport coefficients and constitutive relations [JeRi85, JeRi85b, JeRi85, LuSa84, GoSh95, BrDu98, SeGo96] to yield a hydrodynamic description. Although there is no clear time and length scale separation in systems of granular matter and the range of validity of hydrodynamic methods is still under discussion [TaGo98], the success of a hydrodynamic description for nearly elastic systems is astonishing.

As in standard hydrodynamics, the relevant macroscopic variables are the density, the flow field and the granular temperature. The macroscopic equations commonly used are given by the standard non-linear equations of fluid dynamics, completed by an energy sink term in the transport equation for the energy, which accounts for energy loss due to collisions. A linear stability analysis of these equations around the homogeneous cooling state shows for example the above mentioned instability against shear fluctuations. Fluctuating hydrodynamics [NoEr97, NoBr98, NoEr99] provides a quantitative description of the build up of spatial correlations, which agree well with two-dimensional molecular-dynamics simulations. A mode-coupling-like theory [BrEr98] allows to determine the time dependence of the energy in the time regime where the energy decays much slower then predicted by the homogeneous cooling state due to vortex structures and clustering. 


\subsubsection{Simulational methods}

Simulations of granular matter have become quite important to investigate models and certain experimental set ups which are difficult to be treated analytically. On the other hand simplifying assumptions commonly used in analytical descriptions (as there are for example no gravity, no friction forces, monodispersity of the particles, constant coefficient of restitution) can hardly be realized in experiments, whereas they can easily be realized on the computer. So simulations are a useful tool to gain data, which are hard to access in the real world, to compare with analytical descriptions and to guide the theorist in her considerations.

Mainly three computational methods are used to investigate rapid granular flow: To perform molecular dynamics the forces acting during the contact have to be specified, although they are rarely determined by experiments and have to be introduced in a more phenomenological way. Once the forces are given, one numerically integrates Newton's equations of motion. Here the collisions are not instantaneous and the contact takes a finite time. In event-driven simulations the system is being updated from collision event to collision event. The main assumption is that collisions occur instantaneously and the velocities after collisions are completely determined by the values before collisions. In contrast to molecular dynamics these "collision rules" can be supported by experimental observations [FoLo94]. The direct Monte Carlo method is similar to the event-driven method. The essential difference is that the collisions are dealt with a probabilistic rather than a deterministic basis. This requires the assumption of molecular chaos and restricts the method to dilute granular flow. In ref. [Bi76] it is shown that the basic assumptions are common to the Boltzmann equation and that the result is equivalent to a numerical solution of the Boltzmann equation.

\section{Molecular dynamics}

In a molecular-dynamic simulation Newton's equations of motion are solved numerically [AlTi87, Ve67]. Therefore the corresponding forces which should lead to realistic binary collisions have to be formulated. For an overview see for example [Lu98]. One distinguishes between four types of forces: (i) To model 'hard' particles, a short range, repulsive potential is required. It depends on the elastic properties and on the shape of the particles. (ii) In order to introduce dissipation into the system, one assumes viscous damping, i.e. a force which depends on the relative velocity of the particle and is directed opposite to the velocity. (iii) Tangential friction forces are modeled by viscous forces tangential to the surface of the particles and are responsible for loss of the rotational energy. Another possibility is to formulate Coulomb friction by assuming that the friction force is proportional to the normal force. In many materials the tangential velocity is reversed in a collision, i.e. elastic properties of the material store the kinetic energy of the tangential part of velocity and release it 
again. Therefore (iv) tangential repulsive forces as for the normal direction of point (i) are formulated.

\section{Event driven}

To investigate rapid granular flow, event-driven simulations (ED) are particularly suitable for three reasons: (i) They allow for simulations of large systems and for long times. (ii) They correspond perfectly to the dynamics we describe analytically. (iii) The "collision rules" can be confirmed directly in experiments.

In the simulation the system is propagated from collision event to collision event. In between the particles move freely and deterministically. The main part of the computational work is to search for the next collision event and to update the system. We will describe this simulational technique in detail in section 2.4.1.

\section{Direct simulation Monte Carlo}

The direct simulation Monte Carlo (DSMC) method was first proposed by Bird [Bi76] for the simulation of rarefied gases, and has been applied also in the context of granular matter [BrRu96, MuLu97, BrCu99]. The basic assumption of DSMC is that the movement of the particles and the interaction can be handled one after another. First in a time step $\tau$ the particles are moved according to their equations of motion, without interaction with the other particles. The free movement can either be calculated by a standard numerical integration scheme or by means of the analytical solution of the equations of motion. Second interactions are taken into account. In contrast to ED simulations the exact places and times are not calculated but chosen randomly in a way that the correct dynamics is mirrored. The simulation box is divided into cells. Only the particles in one cell can interact. In each cell with more than one particle a certain number of pairs of particles are chosen randomly from which only a few pairs are selected with a probability, which corresponds to the relative velocity of the particles. The collisions are now performed by choosing again randomly an impact parameter. Here the assumption of molecular chaos enters the theory. Then the post-collisional velocities are calculated as if the two particles have collided with that impact parameter. The advantage of this simulation is that it is fastest and can deal with many more particles then other simulation techniques, but it neglects correlated collisions and is therefore subject to the same restrictions as the Boltzmann equation.

\subsection{Questions to be answered}

The main goal of this work is to understand freely cooling systems of particles with rotational degrees of freedom. Since cooling of smooth spheres is quite well-understood we ask ourselves how the found phenomena are specific for the model. How does 
friction influence the system and what happens if we consider non-spherical particles? We use the smooth spheres as a guideline for our work: We start to investigate the first stage, i.e. the HCS, by means of kinetic theory. How can Haff's law of homogeneous cooling be generalized when rotational degrees of freedom are included? Furthermore it is not at all obvious if one finds a shear and cluster instability also in systems of rough spheres and especially in systems of non-spherical particles. We show by means of computer simulations that this is indeed the case, and in particular we investigate the influence and behavior of the rotational degrees of freedom.

\section{Outline}

In chapter 2 we introduce the models under consideration and describe the dynamics in terms of the time-evolution operator. For a comprehensive understanding we first discuss smooth potentials and recall the formalism of a pseudo-Liouville operator for elastic, hard-core collisions. Subsequently the formalism is extended to inelastic, rough spheres and needles. We briefly describe the event-driven method we used to perform simulations. The homogeneous cooling state is introduced in chapter 3 . We present results for both spheres and needles, assuming a Maxwellian distribution for linear and angular momenta. Corrections to the Gaussian approximation are investigated for smooth and rough spheres. Different time scales of the HCS are discussed in detail. In chapter 4 we show by means of simulations that for dense systems of needles the assumption of homogeneity breaks down. In the second part of chapter 4 we investigate in particular the dynamics of the rotational energy for rough spheres in the regime of inhomogeneous cooling. In chapter 5 we compare an approach using the Enskog Boltzmann equation with the approach introduced in this thesis. Finally, in chapter 6 we summarize results and give an outlook. 


\section{The Liouville operator}

We are interested in macroscopic properties of systems of many particles which are themselves of meso- or macroscopic size, i.e. behave according to the rules of classical mechanics as opposed to quantum mechanics. In addition, the systems are granular so energy is not conserved. This means that they can not be treated by Hamiltonian mechanics. In this chapter we will present a formulation of the dynamics based on the Liouville operator that still enables us to derive properties of the system under consideration.

We consider two different types of systems: The constituents of the first are hard spheres of diameter $d$ and the constituents of the second are (infinitely) thin, hard rods or needles of length $L$. In order to keep the discussion as transparent as possible, the formalism of the (pseudo-)Liouville operator will be demonstrated for Hamiltonian systems with smooth potentials first, for hard-core potentials next, and finally for granular spheres and needles. It is interesting to note that both cases, spheres and needles, are analytically tractable so that comparisons between different geometrical particle shapes are possible.

The dynamics can either be described in terms of the time dependence of observables or in terms of the time dependence of the distribution function. We derive for the case of rough spheres the pseudo-Liouville equation, which describes explicitly the time dependence of the distribution function.

In the last part of this chapter we describe in more detail the event-driven method (ED), which we use to perform simulations and which mimics the dynamics of hardcore systems. 


\subsection{Classical systems}

We review the description of the dynamics of a classical many-particle systems in terms of a Liouville operator. We extend the definition to hard-core interactions and introduce the pseudo-Liouville operator, for which we derive explicit formulas for the cases of rough spheres and needles.

\subsubsection{Smooth potentials}

We consider a system of $N$ classical particles of mass $m$ in a volume $V$, interacting through a pair potential $W$. The system is characterized by its total energy

$$
H=\sum_{i=1}^{N} \frac{\boldsymbol{p}_{i}^{2}}{2 m}+\sum_{i<j} W\left(\boldsymbol{r}_{i}-\boldsymbol{r}_{j}\right)
$$

in terms of particle momenta $\boldsymbol{p}_{i}$ and coordinates $\boldsymbol{r}_{i}$. The time evolution of an observable $f(\Gamma)$, which is a function of phase-space variables $\Gamma:=\left\{\boldsymbol{r}_{i}, \boldsymbol{p}_{i}\right\}$, but does not depend on time explicitly, is given in terms of the Poisson bracket by

$$
\frac{d f}{d t}=\{H, f\}=: i \mathcal{L} f .
$$

This defines the Liouville operator $\mathcal{L}$. The time evolution of $f$ can then formally be written as $\mathcal{L}: f(t)=e^{i \mathcal{L} t} f(0)$.

We decompose the Liouville operator $\mathcal{L}=\mathcal{L}_{0}+\mathcal{L}_{\text {inter }}$ into a free streaming part $\mathcal{L}_{0}$ and an operator $\mathcal{L}_{\text {inter }}$, which accounts for interactions. The definition of the Poisson bracket,

$$
\{H, f\}=\sum_{j}\left(\frac{\partial f}{\partial \boldsymbol{r}_{j}} \frac{\partial H}{\partial \boldsymbol{p}_{j}}-\frac{\partial f}{\partial \boldsymbol{p}_{j}} \frac{\partial H}{\partial \boldsymbol{r}_{j}}\right)
$$

thus yields

$$
i \mathcal{L}_{0}=\sum_{j} i \mathcal{L}_{0}^{j}=\sum_{j} \frac{\boldsymbol{p}_{j}}{m} \frac{\partial}{\partial \boldsymbol{r}_{j}} \quad \text { and } \quad i \mathcal{L}_{\text {inter }}=\sum_{j<k} \frac{\partial W}{\partial \boldsymbol{r}_{j k}}\left(\frac{\partial}{\partial \boldsymbol{p}_{k}}-\frac{\partial}{\partial \boldsymbol{p}_{j}}\right) .
$$

\subsubsection{Elastic hard-core interactions}

A pseudo-Liouville operator for hard-core collisions has been formulated by Ernst et al. [ErDo69] and has been applied by many groups [ReLe75, Re75, Le82] to study the dynamic evolution of a gas of hard spheres. Collisions are instantaneous and characterized by collision rules. In a collision of two particles, numbered 1 and 2 , 
their pre-collisional velocities $\boldsymbol{v}_{1}=\boldsymbol{p}_{1} / m$ and $\boldsymbol{v}_{2}=\boldsymbol{p}_{2} / m$ are changed instantaneously to their post-collisional values $\boldsymbol{v}_{1}^{\prime}$ and $\boldsymbol{v}_{2}^{\prime}$ according to

$$
\begin{aligned}
& \boldsymbol{v}_{1}^{\prime}=\boldsymbol{v}_{1}-\left(\boldsymbol{v}_{12} \hat{\boldsymbol{r}}_{12}\right) \hat{\boldsymbol{r}}_{12} \\
& \boldsymbol{v}_{2}^{\prime}=\boldsymbol{v}_{2}+\left(\boldsymbol{v}_{12} \hat{\boldsymbol{r}}_{12}\right) \hat{\boldsymbol{r}}_{12} .
\end{aligned}
$$

We have denoted the relative velocity by $\boldsymbol{v}_{12}=\boldsymbol{v}_{1}-\boldsymbol{v}_{2}$, and $\hat{\boldsymbol{r}}_{12}=\boldsymbol{r}_{12} /\left|\boldsymbol{r}_{12}\right|$, with $\boldsymbol{r}_{12}=\boldsymbol{r}_{1}-\boldsymbol{r}_{2}$. The free-streaming part $\mathcal{L}_{0}$ of the Liouville-operator remains unchanged, whereas the interaction part $\mathcal{L}_{\text {inter }}$ has to be modified because the potential is no longer differentiable in the limit of hard-core interactions. It turns out that $\mathcal{L}$ is no longer hermitian as it is for systems with smooth potentials. This is why it is called a pseudo-Liouville operator for hard-core systems.

In order to construct the pseudo-Liouville operator, we consider the change of a dynamical variable due to a collision of just two particles. What we need is an operator $\mathcal{T}_{+}^{(12)}$ that

- generates the change of an observable caused by a collision when integrated over a short time interval containing the collision time,

- only acts at the time of contact,

- only acts when the particles are approaching, but not when they are receding.

The second requirement can be satisfied by the ansatz $\mathcal{T}_{+}^{(12)} \propto \delta\left(\left|\boldsymbol{r}_{12}\right|-d\right)$, the third one demands $\mathcal{T}_{+}^{(12)} \propto \Theta\left(-\frac{d}{d t}\left|\boldsymbol{r}_{12}\right|\right)$, where $\Theta(\cdot)$ is the usual Heaviside step function. In order to satisfy the first requirement, we introduce an operator $b_{+}^{(12)}$ which is defined by its action on an observable $f$ according to

$$
b_{+}^{(12)} f\left(\boldsymbol{v}_{1}, \boldsymbol{v}_{2}\right)=f\left(\boldsymbol{v}_{1}^{\prime}, \boldsymbol{v}_{2}^{\prime}\right),
$$

i.e. it simply replaces all velocities according to eqs. (2.5). The operator $\mathcal{T}_{+}^{(12)}$ should generate the change caused by a collision so that $\mathcal{T}_{+}^{(12)} \propto b_{+}^{(12)}-1$. We collect the three terms and make sure to include a prefactor which is chosen such that the integration of an observable over a short time interval around the collision time yields the change of the observable, as induced by the collision rules (2.5). The complete expression for $\mathcal{T}_{+}^{(12)}$ is thus

$$
i \mathcal{T}_{+}^{(12)}=\left|\frac{d}{d t}\right| \boldsymbol{r}_{12}|| \delta\left(\left|\boldsymbol{r}_{12}\right|-d\right) \Theta\left(-\frac{d}{d t}\left|\boldsymbol{r}_{12}\right|\right)\left(b_{+}^{(12)}-1\right)
$$

Since the probability that three or more particles touch at precisely the same instant is zero, we only need to consider two particle collisions and find for the time-evolution 
operator for the system of elastically colliding hard spheres:

$$
\begin{aligned}
\mathcal{L} & =\mathcal{L}_{0}+\Theta(t) \mathcal{L}_{+}+\Theta(-t) \mathcal{L}_{-} \text {with } \\
i \mathcal{L}_{ \pm} & =\sum_{i<j} i \mathcal{T}_{ \pm}^{(i j)}=\sum_{i<j}\left|\frac{d}{d t}\right| \boldsymbol{r}_{j i}|| \delta\left(\left|\boldsymbol{r}_{j i}\right|-d\right) \Theta\left(\mp \frac{d}{d t}\left|\boldsymbol{r}_{j i}\right|\right)\left(b_{ \pm}^{(i j)}-1\right)
\end{aligned}
$$

The negative time evolution is given by $\mathcal{L}_{-}$, and $b_{-}^{(i j)}$ is the operator that replaces post-collisional velocities by pre-collisional ones.

\section{Extension to rough spheres}

Models of elastically colliding hard-core spheres have been extended to include rotational degrees of freedom and surface roughness [JeRi85, JeRi85b, ChCo60]. Rotational degrees of freedom offer the possibility to describe molecules with internal degrees of freedom and surface roughness is needed to transfer energy from the translational degrees of freedom to the rotational ones.

We only discuss the simplest case of identical spheres of mass $m$, moment of inertia $I$ and diameter $d$. Translational motion is characterized by the center of mass velocities $\boldsymbol{v}_{i}$ and rotational motion by the angular velocities $\boldsymbol{\omega}_{i}$. Let the surface normal $\hat{\boldsymbol{r}}_{12}$ at the point of contact point from sphere 2 to sphere 1 . The important quantity to model the collision is the relative velocity of the point of contact:

$$
\boldsymbol{V}=\left(\boldsymbol{v}_{1}-\frac{d}{2} \boldsymbol{\omega}_{1} \times \hat{\boldsymbol{r}}_{12}\right)-\left(\boldsymbol{v}_{2}+\frac{d}{2} \boldsymbol{\omega}_{2} \times \hat{\boldsymbol{r}}_{12}\right)
$$

There are two contributions, first the center-of-mass velocity of each sphere, and second the contributions from the rotations of each sphere. The minus sign in the first parenthesis stems from the fact that the surface normal $\hat{\boldsymbol{r}}_{12}$, as it was defined, points outwards for sphere 2 and inwards for sphere 1.

Now we can specify the collision rules. Primed variables always denote quantities immediately after the collision; unprimed variables denote pre-collisional quantities:

$$
\begin{aligned}
\hat{\boldsymbol{r}}_{12} \boldsymbol{V}^{\prime} & =-\hat{\boldsymbol{r}}_{12} \boldsymbol{V}, \\
\hat{\boldsymbol{r}}_{12} \times \boldsymbol{V}^{\prime} & =-\hat{\boldsymbol{r}}_{12} \times \boldsymbol{V} .
\end{aligned}
$$

Eqs. (2.11) constitute three linearly independent equations. In addition, total momentum is conserved,

$$
\boldsymbol{v}_{1}^{\prime}+\boldsymbol{v}_{2}^{\prime}=\boldsymbol{v}_{1}+\boldsymbol{v}_{2},
$$

and forces during a collision can only act at the point of contact. Therefore there is no torque with respect to this point and consequently we have conserved angular 
momentum (also with respect to the point of contact) for both particles involved:

$$
\begin{aligned}
& \frac{m d}{2} \hat{\boldsymbol{r}}_{12} \times\left(\boldsymbol{v}_{1}^{\prime}-\boldsymbol{v}_{1}\right)+I\left(\boldsymbol{\omega}_{1}^{\prime}-\boldsymbol{\omega}_{1}\right)=\mathbf{0} \\
& \frac{m d}{2} \hat{\boldsymbol{r}}_{12} \times\left(\boldsymbol{v}_{2}^{\prime}-\boldsymbol{v}_{2}\right)-I\left(\boldsymbol{\omega}_{2}^{\prime}-\boldsymbol{\omega}_{2}\right)=\mathbf{0} .
\end{aligned}
$$

Altogether we have 12 independent equations for 12 unknowns, namely the four vectors $\boldsymbol{v}_{i}^{\prime}$ and $\boldsymbol{\omega}_{i}^{\prime}, i=1,2$, with three components each. Solving for these, we obtain:

$$
\begin{aligned}
\boldsymbol{v}_{1}^{\prime} & =\boldsymbol{v}_{1}-\eta_{t} \boldsymbol{v}_{12}-\left(\eta_{n}-\eta_{t}\right)\left(\hat{\boldsymbol{r}}_{12} \boldsymbol{v}_{12}\right) \hat{\boldsymbol{r}}_{12}-\eta_{t} \frac{d}{2} \hat{\boldsymbol{r}}_{12} \times\left(\boldsymbol{\omega}_{1}+\boldsymbol{\omega}_{2}\right) \\
\boldsymbol{v}_{2}^{\prime} & =\boldsymbol{v}_{2}+\eta_{t} \boldsymbol{v}_{12}+\left(\eta_{n}-\eta_{t}\right)\left(\hat{\boldsymbol{r}}_{12} \boldsymbol{v}_{12}\right) \hat{\boldsymbol{r}}_{12}+\eta_{t} \frac{d}{2} \hat{\boldsymbol{r}}_{12} \times\left(\boldsymbol{\omega}_{1}+\boldsymbol{\omega}_{2}\right) \\
\boldsymbol{\omega}_{1}^{\prime} & =\boldsymbol{\omega}_{1}+\frac{2}{d q} \eta_{t} \hat{\boldsymbol{r}}_{12} \times \boldsymbol{v}_{12}+\frac{\eta_{t}}{q} \hat{\boldsymbol{r}}_{12} \times\left(\hat{\boldsymbol{r}}_{12} \times\left(\boldsymbol{\omega}_{1}+\boldsymbol{\omega}_{2}\right)\right) \\
\boldsymbol{\omega}_{2}^{\prime} & =\boldsymbol{\omega}_{2}+\frac{2}{d q} \eta_{t} \hat{\boldsymbol{r}}_{12} \times \boldsymbol{v}_{12}+\frac{\eta_{t}}{q} \hat{\boldsymbol{r}}_{12} \times\left(\hat{\boldsymbol{r}}_{12} \times\left(\boldsymbol{\omega}_{1}+\boldsymbol{\omega}_{2}\right)\right) .
\end{aligned}
$$

The dimensionless constant $q=4 I /\left(m d^{2}\right)$ abbreviates a frequently appearing combination of factors. We have also introduced two parameters $\eta_{n}$ and $\eta_{t}$, because we anticipate the more general collision rules for the inelastic case. For elastically colliding, perfectly rough spheres, we simply have $\eta_{n}=1$ and $\eta_{t}=q /(1+q)$.

The pseudo-Liouville operator for elastically colliding rough spheres is still given by eq. (2.9) but the operator $b_{+}^{(i j)}$ now replaces linear and angular velocities according to eqs. (2.14).

\section{Extension to rough needles}

Elastic collisions of hard needles have been discussed by Frenkel et al. [FrMa83]. It is straightforward to rephrase their results in terms of a pseudo-Liouville operator [HuAs99]. The free-streaming part of the Liouville operator is derived from the kinetic energy of the Hamiltonian according to the general rules of classical mechanics. Note, however, that for thin needles, one of the moments of inertia is zero; this implies that the angular-momentum component along the corresponding axis, which points along the orientation of the needle, is also always zero. Therefore, rotations about this axis can be ignored, and $\boldsymbol{\omega}$ has only two components, both perpendicular to the orientation of the needle. The center-of-mass coordinate of needle $i$ will be denoted by $\boldsymbol{r}_{i}$ and its orientation by the unit vector $\boldsymbol{u}_{i}$. The moments of inertia perpendicular to $\boldsymbol{u}_{i}$ are equal due to symmetry and will be denoted by $I$.

The formulation of the collision rules proceeds in close analogy to the case of rough spheres. First we determine the conditions of contact. The unit vectors $\boldsymbol{u}_{1}$ 
and $\boldsymbol{u}_{2}$ span a plane $E_{12}$ with normal

$$
\boldsymbol{u}_{\perp}=\frac{\boldsymbol{u}_{1} \times \boldsymbol{u}_{2}}{\left|\boldsymbol{u}_{1} \times \boldsymbol{u}_{2}\right|} .
$$

We decompose $\boldsymbol{r}_{12}=\boldsymbol{r}_{1}-\boldsymbol{r}_{2}$ into a component perpendicular $\boldsymbol{r}_{12}^{\perp}=\left(\boldsymbol{r}_{12} \boldsymbol{u}_{\perp}\right) \boldsymbol{u}_{\perp}$ and parallel $\boldsymbol{r}_{12}^{\|}=\left(s_{12} \boldsymbol{u}_{1}-s_{21} \boldsymbol{u}_{2}\right)$ to $E_{12}$ (see fig. 2.1). The hard rods are in contact if $\boldsymbol{r}_{12} \boldsymbol{u}^{\perp}=0$ and simultaneously $\left|s_{12}\right|<L / 2$ and $\left|s_{21}\right|<L / 2$. The relative velocity of

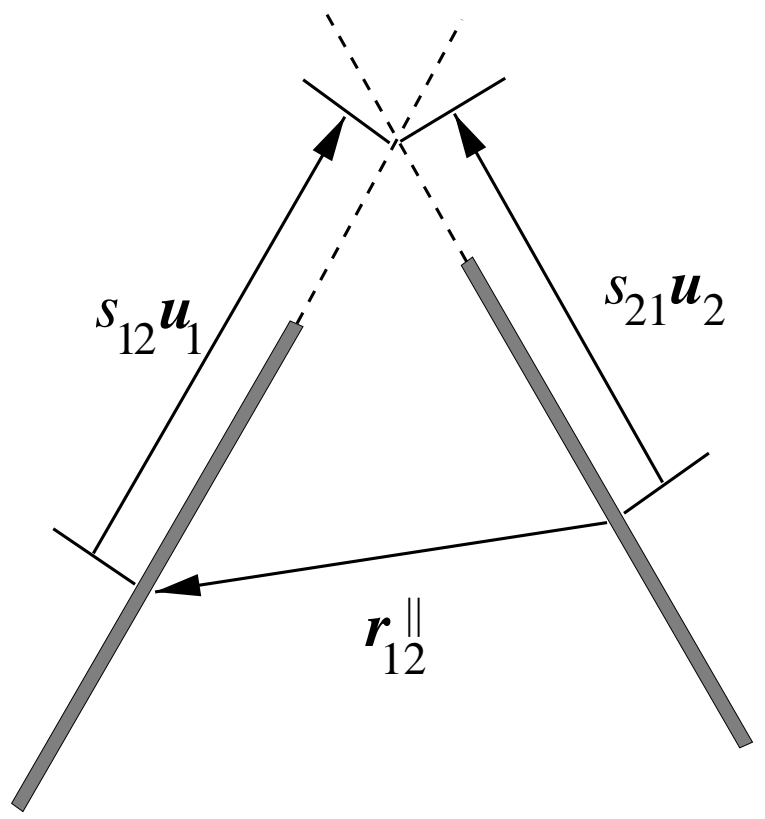

Figure 2.1.: Configuration of two needles projected into the plane spanned by the unit vectors $\boldsymbol{u}_{1}$ and $\boldsymbol{u}_{2}$.

the point of contact is given by

$$
\boldsymbol{V}=\boldsymbol{v}_{12}+s_{12} \dot{\boldsymbol{u}}_{1}-s_{21} \dot{\boldsymbol{u}}_{2} .
$$

It is useful to introduce a set of normalized basis vectors

$$
\boldsymbol{u}_{1}, \quad \boldsymbol{u}_{1}^{\perp}=\left(\boldsymbol{u}_{2}-\left(\boldsymbol{u}_{1} \boldsymbol{u}_{2}\right) \boldsymbol{u}_{1}\right) / \sqrt{1-\left(\boldsymbol{u}_{1} \boldsymbol{u}_{2}\right)^{2}}, \quad \text { and } \quad \boldsymbol{u}_{\perp}
$$

with $\boldsymbol{u}_{\perp}$ defined in eq. (2.15). Total momentum conservation is given by (2.12) and conservation of angular momentum with respect to the contact point reads

$$
\boldsymbol{\omega}_{1}^{\prime}=\boldsymbol{\omega}_{1}+\frac{m s_{12}}{I} \boldsymbol{u}_{1} \times\left(\boldsymbol{v}_{1}^{\prime}-\boldsymbol{v}_{1}\right) \quad \text { and } \quad \boldsymbol{\omega}_{2}^{\prime}=\boldsymbol{\omega}_{2}+\frac{m s_{21}}{I} \boldsymbol{u}_{2} \times\left(\boldsymbol{v}_{2}^{\prime}-\boldsymbol{v}_{2}\right) .
$$

Three additional equations follow from the change in the relative velocity of the contact point, which is modeled in close analogy to the case of rough spheres:

$$
\boldsymbol{V} \boldsymbol{u}_{\perp}=-\boldsymbol{V} \boldsymbol{u}_{\perp}, \quad \boldsymbol{V} \boldsymbol{u}_{1}=-e_{t} \boldsymbol{V} \boldsymbol{u}_{1}, \quad \text { and } \quad \boldsymbol{V} \boldsymbol{u}_{2}=-e_{t} \boldsymbol{V} \boldsymbol{u}_{2} .
$$


Again, energy conservation implies $e_{t}= \pm 1$, corresponding to either perfectly rough or perfectly smooth needles (see also eq. (2.32)). Solving for $\boldsymbol{v}_{i}^{\prime}$ and $\boldsymbol{\omega}_{i}^{\prime}$, we obtain after a lengthy calculation:

$$
\boldsymbol{v}_{1}^{\prime}=\boldsymbol{v}_{1}+\Delta \boldsymbol{v}, \quad \boldsymbol{v}_{2}^{\prime}=\boldsymbol{v}_{2}-\Delta \boldsymbol{v}
$$

$\boldsymbol{\omega}_{1}^{\prime}, \boldsymbol{\omega}_{2}^{\prime}$ given by eq. (2.18) and the change in velocity $\Delta \boldsymbol{v}$ can be decomposed with respect to the basis defined above, $\Delta \boldsymbol{v}=\gamma_{1} \boldsymbol{u}_{1}+\gamma_{2} \boldsymbol{u}_{1}^{\perp}+\alpha \boldsymbol{u}_{\perp}$. The coefficient $\alpha$ is given by

$$
\alpha=-\left(1+\frac{m s_{12}^{2}}{2 I}+\frac{m s_{21}^{2}}{2 I}\right)^{-1} \boldsymbol{V} \boldsymbol{u}_{\perp}
$$

while $\gamma_{1}$ and $\gamma_{2}$ satisfy the set of linear equations

$$
\left(\begin{array}{ll}
A & B \\
B & C
\end{array}\right)\left(\begin{array}{l}
\gamma_{1} \\
\gamma_{2}
\end{array}\right)=-\frac{1+e_{t}}{2}\left(\begin{array}{c}
\boldsymbol{V} \boldsymbol{u}_{1} \\
\boldsymbol{V} \boldsymbol{u}_{1}^{\perp}
\end{array}\right)
$$

with

$$
\begin{aligned}
& A=1+\frac{m s_{21}^{2}}{2 I}\left(1-\left(\boldsymbol{u}_{1} \boldsymbol{u}_{2}\right)^{2}\right), \\
& B=-\frac{m s_{21}^{2}}{2 I}\left(\boldsymbol{u}_{1} \boldsymbol{u}_{2}\right) \sqrt{1-\left(\boldsymbol{u}_{1} \boldsymbol{u}_{2}\right)^{2}}, \\
& C=1+\frac{m s_{12}^{2}}{2 I}+\frac{m s_{21}^{2}}{2 I}\left(\boldsymbol{u}_{1} \boldsymbol{u}_{2}\right)^{2} .
\end{aligned}
$$

The Liouville operator for two needles must obey the same basic requirements as for spheres. The only changes are in the condition for a collision to take place ${ }^{1}$,

$$
i \mathcal{T}_{+}^{(12)} \propto \Theta\left(L / 2-\left|s_{12}\right|\right) \Theta\left(L / 2-\left|s_{21}\right|\right) \delta\left(\left|\boldsymbol{r}_{12}^{\perp}\right|-0^{+}\right),
$$

and in the condition that the two particles are approaching,

$$
i \mathcal{T}_{+}^{(12)} \propto \Theta\left(-\frac{d}{d t}\left|\boldsymbol{r}_{12}^{\perp}\right|\right)
$$

Collecting the terms and choosing the correct prefactor gives the result

$$
\begin{aligned}
i \mathcal{T}_{+}^{(12)}=\left|\frac{d}{d t}\right| \boldsymbol{r}_{12}^{\perp}|| \Theta( & \left.-\frac{d}{d t}\left|\boldsymbol{r}_{12}^{\perp}\right|\right) \times \\
& \Theta\left(L / 2-\left|s_{12}\right|\right) \Theta\left(L / 2-\left|s_{21}\right|\right) \delta\left(\left|\boldsymbol{r}_{12}^{\perp}\right|-0^{+}\right)\left(b_{+}^{(12)}-1\right) .
\end{aligned}
$$

The operator $b_{+}^{(12)}$ replaces all velocities according to eqs. (2.20).

\footnotetext{
${ }^{1}$ We assume the needles to be infinitely thin hard rods, i.e. $\left|\boldsymbol{r}_{12}^{\perp}\right|=0$ at contact. The quantity $\left|\boldsymbol{r}_{12}^{\perp}(t)\right|$ is not differentiable at times $t_{0}$, for which $\left|\boldsymbol{r}_{12}^{\perp}\left(t_{0}\right)\right|=0$, but we have to take the time derivative at the point of contact. Therefore we take the limit of arbitrarily but finitely thin lines, with thickness denoted by $0^{+}$. It should be read as $\delta\left(\left|\boldsymbol{r}_{12}^{\perp}\right|-0^{+}\right)=\lim _{\epsilon \backslash 0} \delta\left(\left|\boldsymbol{r}_{12}^{\perp}\right|-\epsilon\right)$. This definition will become important in the calculations and will help us to distinguish if one particle touches the other from 'above' or 'below'. See also footnote 1 in appendix A.2.
} 


\subsection{Inelastic collision}

The collision rules for rough spheres and needles are easily generalized to the case of inelastic collisions. This will allow us to set up a formulation of the dynamics of inelastically colliding grains in terms of a pseudo-Liouville operator.

\subsubsection{Rough spheres}

Energy dissipation is modeled by normal and tangential restitution, see e.g. [JeRi85, LuSa87, Ce95]. The collision rules imply for the change in the relative velocity of the points of contact:

$$
\begin{aligned}
\hat{\boldsymbol{r}}_{12} \boldsymbol{V}^{\prime} & =-e_{n} \hat{\boldsymbol{r}}_{12} \boldsymbol{V} \\
\hat{\boldsymbol{r}}_{12} \times \boldsymbol{V}^{\prime} & =-e_{t} \hat{\boldsymbol{r}}_{12} \times \boldsymbol{V} .
\end{aligned}
$$

The first equation describes the reduction of the normal-velocity component by a nonnegative factor $e_{n}$. This is the well-known normal restitution. The second equation is intended to describe surface roughness and friction in that it imposes a reduction or even a reversal of the tangential-velocity component. This is motivated by the picture of small "bumps" on the surface which become hooked when the surfaces are very close. For all $-1<e_{t}<+1$ dissipation is present.

The change in energy in a collision is given by

$$
\begin{aligned}
\Delta E=-m\left(\frac{1-e_{n}^{2}}{4}\left(\hat{\boldsymbol{r}}_{12} \boldsymbol{v}_{12}\right)^{2}+\right. & \\
& \left.\frac{1-e_{t}^{2}}{4} \frac{q}{1+q}\left(\boldsymbol{v}_{12}-\left(\hat{\boldsymbol{r}}_{12} \boldsymbol{v}_{12}\right) \hat{\boldsymbol{r}}_{12}-\frac{d}{2} \hat{\boldsymbol{r}}_{12} \times\left(\boldsymbol{\omega}_{1}+\boldsymbol{\omega}_{2}\right)\right)^{2}\right) .
\end{aligned}
$$

With the parameter range $0 \leq e_{n} \leq 1$ and $-1 \leq e_{t} \leq 1$, energy is only lost and never gained in a single collision.

The conservation laws for linear and angular momenta are unchanged, so we obtain the same set of equations for the post-collisional velocities as eqs. (2.14), with, however, different parameter values

$$
\eta_{n}=\frac{1+e_{n}}{2} \quad \text { and } \quad \eta_{t}=\frac{q}{1+q} \frac{e_{t}+1}{2} .
$$

Later we will need the inversion of eqs. (2.14), i.e. for given post-collisional velocities we want to determine the pre-collisional ones. This is simply done by replacing $e_{t}$ by $1 / e_{t}$ and $e_{n}$ by $1 / e_{n}$ in eqs. (2.14). The pre-collisional velocities obtained from post-collisional ones will be denoted by $\boldsymbol{v}_{1}^{\prime \prime}, \boldsymbol{v}_{2}^{\prime \prime}, \boldsymbol{\omega}_{1}^{\prime \prime}$ and $\boldsymbol{\omega}_{2}^{\prime \prime}$ in the following. 


\subsubsection{Rough needles}

For hard needles we introduce normal and tangential restitution according to

$$
\boldsymbol{V}^{\prime} \boldsymbol{u}_{\perp}=-e_{n} \boldsymbol{V} \boldsymbol{u}_{\perp}, \quad \boldsymbol{V}^{\prime} \boldsymbol{u}_{1}=-e_{t} \boldsymbol{V} \boldsymbol{u}_{1}, \quad \text { and } \quad \boldsymbol{V}^{\prime} \boldsymbol{u}_{2}=-e_{t} \boldsymbol{V} \boldsymbol{u}_{2} .
$$

The conservation laws for linear and angular momenta are the same as for the elastic case so that one arrives at the same set of eqs. (2.20), the only change affecting the parameter

$$
\alpha=-\frac{1+e_{n}}{2}\left(1+\frac{m s_{12}^{2}}{2 I}+\frac{m s_{21}^{2}}{2 I}\right)^{-1} \boldsymbol{V} \boldsymbol{u}_{\perp} .
$$

The energy loss in a collision of two needles is given by

$$
\begin{aligned}
& \Delta E=-m \frac{1-e_{t}^{2}}{4}\left(\frac{C\left(\boldsymbol{V} \boldsymbol{u}_{1}\right)^{2}-2 B\left(\boldsymbol{V} \boldsymbol{u}_{1}\right)\left(\boldsymbol{V} \boldsymbol{u}_{1}^{\perp}\right)+A\left(\boldsymbol{V} \boldsymbol{u}_{1}^{\perp}\right)^{2}}{A C-B^{2}}\right) \\
&-m \frac{1-e_{n}^{2}}{4}\left(1+\frac{m s_{12}^{2}}{2 I}+\frac{m s_{21}^{2}}{2 I}\right)^{-1}\left(\boldsymbol{V} \boldsymbol{u}_{\perp}\right)^{2} .
\end{aligned}
$$

It can be checked with eqs. (2.23) that the first term is non-positive if and only if $-1 \leq e_{t} \leq 1$. Obviously, the second term is also non-positive if $0 \leq e_{n} \leq 1$. Our method of modeling granular collisions of needles is therefore consistent with the constraint that energy may not be gained in a single collision.

\subsection{Time evolution of the distribution function}

The time evolution of the distribution function is derived for rough spheres. The corresponding time-evolution operator of the distribution function is calculated.

We will be interested in phase-space averages of an observable $f(\Gamma)$ at a time $t$ defined by:

$$
\langle f\rangle(t)=\int d \Gamma \rho(\Gamma ; 0) f(\Gamma ; t)=\int d \Gamma \rho(\Gamma ; t) f(\Gamma) .
$$

Here $\rho(\Gamma ; t)$ is the $N$-particle distribution function at time $t$. The average can either be taken over the initial distribution $\rho(\Gamma ; 0)$ at time 0 , with the observable being propagated to time $t$, or equivalently over the distribution $\rho(\Gamma ; t)$ at time $t$ with the unchanged observable $f(\Gamma)$. We write eq. (2.33) $\mathrm{as}^{2}$

$$
\langle f\rangle(t)=\int d \Gamma \rho(\Gamma ; 0) e^{i \mathcal{L} t} f(\Gamma)=: \int d \Gamma\left(e^{i \overline{\mathcal{L}} t} \rho(\Gamma ; 0)\right) f(\Gamma),
$$

\footnotetext{
${ }^{2}$ It was shown in [NoEr98] that the time-evolution operator of the $N$-particle system can be represented in the form $\exp (i \overline{\mathcal{L}} t)$ without generating overlap configurations.
} 
to define the time-evolution operator $\overline{\mathcal{L}}$ which describes the time evolution of $\rho$. To determine $\overline{\mathcal{L}}$ explicitly, we take the derivative of eq. (2.34) at time $t=0$ for simplicity,

$$
\begin{aligned}
\left.\partial_{t}\langle f\rangle(t)\right|_{t=0} & =\int d \Gamma \rho(\Gamma ; 0) i \mathcal{L} f(\Gamma) \\
& =\int d \Gamma\left(\left.\partial_{t} \rho(\Gamma ; t)\right|_{t=0}\right) f(\Gamma)=\int d \Gamma(i \overline{\mathcal{L}} \rho(\Gamma ; 0)) f(\Gamma)
\end{aligned}
$$

The time-evolution operator of the density due to free streaming, $\overline{\mathcal{L}}_{0}$, is easily calculated by partial integration and we get $\overline{\mathcal{L}}_{0}=-\mathcal{L}_{0}$. To find an expression for the time-evolution operator of the density due to collisions $\overline{\mathcal{T}}_{+}^{(12)}$ for spheres, we use eq. (2.35). Phase-space coordinates before collision are denoted by $\Gamma$, after collision by $\Gamma^{\prime}=b_{+}^{(12)} \Gamma$ so that

$$
\begin{aligned}
& \int d \Gamma \rho(\Gamma ; 0) i \mathcal{T}_{+}^{(12)} f(\Gamma)= \\
& \qquad d \Gamma \rho(\Gamma ; 0) \delta\left(\left|\boldsymbol{r}_{12}\right|-d\right) \Theta\left(-\frac{d}{d t}\left|\boldsymbol{r}_{12}\right|\right)\left|\frac{d}{d t}\right| \boldsymbol{r}_{12}||\left(f\left(\Gamma^{\prime}\right)-f(\Gamma)\right) .
\end{aligned}
$$

In the first term on the right-hand-side we make a coordinate transformation to the variables after collision with Jacobian $\mathcal{J}:=\left|\frac{\partial \Gamma}{\partial \Gamma^{\prime}}\right|$. We use the inverse operator of $b_{+}^{(12)}$, namely $b_{-}^{(12)} \Gamma^{\prime}=\Gamma^{\prime \prime}$. Here the coordinates before collision in terms of the coordinates after collision are denoted by $\Gamma^{\prime \prime}=\Gamma\left(\Gamma^{\prime}\right)$. We note that $\frac{d}{d t}\left|\boldsymbol{r}_{12}\right|=\boldsymbol{v}_{12} \hat{\boldsymbol{r}}_{12}$ and rewrite the first term

$$
\begin{aligned}
& \int d \Gamma \rho(\Gamma ; 0) \delta\left(\left|\boldsymbol{r}_{12}\right|-d\right) \Theta\left(-\frac{d}{d t}\left|\boldsymbol{r}_{12}\right|\right)\left|\frac{d}{d t}\right| \boldsymbol{r}_{12}|| f\left(\Gamma^{\prime}\right)= \\
& \int d \Gamma^{\prime} \mathcal{J} \rho\left(\Gamma^{\prime \prime} ; t\right) \delta\left(\left|\boldsymbol{r}_{12}\right|-a\right) \Theta\left(-\boldsymbol{v}_{12}^{\prime \prime} \hat{\boldsymbol{r}}_{12}\right)\left|\boldsymbol{v}_{12}^{\prime \prime} \hat{\boldsymbol{r}}_{12}\right| f\left(\Gamma^{\prime}\right)
\end{aligned}
$$

Next we rename $\Gamma^{\prime}$ by $\Gamma$ and make use of $\boldsymbol{v}_{n m}^{\prime \prime} \hat{\boldsymbol{r}}_{n m}=-\frac{1}{e_{n}}\left(\boldsymbol{v}_{n m} \hat{\boldsymbol{r}}_{n m}\right)$. This allows us to identify the time-evolution operator of the distribution function, $\overline{\mathcal{T}}_{+}^{(12)}$, by:

$$
i \overline{\mathcal{T}}_{+}^{(12)}=\delta\left(\left|\boldsymbol{r}_{12}\right|-d\right)\left|\frac{d}{d t}\right| \boldsymbol{r}_{12}||\left(\Theta\left(\frac{d}{d t}\left|\boldsymbol{r}_{12}\right|\right) \frac{\mathcal{J}}{e_{n}} b_{-}^{(12)}-\Theta\left(-\frac{d}{d t}\left|\boldsymbol{r}_{12}\right|\right)\right)
$$

It is common to multiply the right-hand-side of eq. (2.38) by $\int d \boldsymbol{\sigma} \delta\left(\boldsymbol{\sigma}-\boldsymbol{r}_{12}\right)$ so that we can replace $\boldsymbol{r}_{12}$ by $\boldsymbol{\sigma}$ in eq. (2.38). In the second term the integral transformation $\boldsymbol{\sigma} \rightarrow-\boldsymbol{\sigma}$ is performed and we integrate over $|\boldsymbol{\sigma}|$. We obtain in $D$ dimensions

$$
i \overline{\mathcal{T}}_{+}^{(12)}=d^{D-1} \int_{\boldsymbol{v}_{12} \hat{\boldsymbol{\sigma}}>0} d \hat{\sigma}\left(\boldsymbol{v}_{12} \hat{\boldsymbol{\sigma}}\right)\left(\frac{\mathcal{J}}{e_{n}} \delta\left(\boldsymbol{r}_{12}-d \hat{\boldsymbol{\sigma}}\right) b_{-}^{(12)}-\delta\left(\boldsymbol{r}_{12}+d \hat{\boldsymbol{\sigma}}\right)\right) .
$$


Finally, we note that $t=0$ is not special since we have only chosen it for the sake of simplicity. Hence we have derived the time-evolution operator for the $N$ particle distribution function $\rho(\Gamma ; t)$ which is given by the pseudo-Liouville equation

$$
\partial_{t} \rho(\Gamma, t)=i\left(-\mathcal{L}_{0}(\Gamma)+\sum_{i<j} \overline{\mathcal{T}}_{+}^{(i j)}\right) \rho(\Gamma, t) .
$$

A similar procedure yields the time-evolution operator for the distribution of needles.

For later purpose we remark the frequently used identity

$$
\frac{d}{d t}\langle f\rangle(t)=\int d \Gamma\left(\frac{d}{d t} \rho(\Gamma, t)\right) f(\Gamma)=\int d \Gamma(i \overline{\mathcal{L}} \rho(\Gamma, t)) f(\Gamma)=\int d \Gamma \rho(\Gamma, t) i \mathcal{L} f(\Gamma) .
$$

\subsection{Event-driven simulations}

The event-driven method is introduced. Tricks to fasten the algorithm are presented, and the peculiarities of needles are discussed.

\subsubsection{General ideas}

In the models we use the particles follow an unperturbed translational motion until a collision occurs. These collisions are binary and the pre-collisional velocities are changed instantaneously to their post-collisional values. In the simplest algorithm, which mimics this dynamics, one computes the time of the next collision, propagates the whole system until this time and updates the velocities of the two colliding particles. This method is straightforward, but inefficient for large numbers of particles for two reasons. First it is not necessary to update the whole system after each event and second we do not need to determine all possible collision times for all pairs of particles (especially in the case of needles, where we have to do this numerically, this is a huge waste of time). Therefore a good algorithm for needles avoids updating of the whole system and restricts the search for collision partners in the neighborhood. We will describe this in detail:

\section{The algorithm of Lubachevsky}

In ref. [Lub91] an event-driven (ED) algorithm was introduced which updates only those two particles which were involved in the last collision. For this a double buffering data structure is implemented, which contains the 'old' status and the 'new' status, each consisting of: time of event, position, velocities, and event-partner.

In the 'new' status of a particle the event is stored, which would be its very next from its point of view, i.e. if no collisions of other particles with its expected 
event-partner crosses its plans. This can happen if earlier collisions of other particles have the effect that the expected partner will collide with another particle first. Nevertheless, the main property of this algorithm is that the earliest collision time stored in all 'new' status is indeed the time for the next collisions of the whole system to be simulated. This ensures that we develop the system from the next 'real' event to the next following 'real' event. The collision times of the 'new' status, are stored in an ordered heap tree such that the next event is found at the top of the heap with computational effort of $\mathcal{O}(1)$; changing the position of one particle in the tree from the top to a new position takes $\mathcal{O}(\log N)$ operations.

When a collision occurs, the 'old' and 'new' status of the participating particles are exchanged. Thus, the former 'new' status becomes the actual 'old' one, while the former 'old' status becomes the 'new' one and is free for future calculations. This seemingly complicated exchange of information is carried out extremely simple and fast by only exchanging the pointers to the 'new' and 'old' status respectively. The 'old' status of particle $i$ has to be kept in memory, in order to calculate the time of the next contact, $t_{i j}$, of particle $i$ with any other object $j$ which can change its status due to a collision with yet another particle. During the simulation this may be necessary several times so that the predicted 'new' status has to be modified. The minimum of all $t_{i j}$ is stored in the 'new' status of particle $i$, together with the corresponding partner $j$ as well as positions and velocities after the collision.

\section{The linked-cell structure}

The search for possible collision partners is accelerated by the use of a standard linked-cell data structure and consumes $\mathcal{O}(1)$ numerical resources [AlTi87]. Then crossing the boundary of such a cell is treated as an event. In total, this results in numerical effort of $\mathcal{O}(N \log N)$ for $N$ particles. For a detailed description of the algorithm see ref. [Lub91].

\section{Inelastic collapse}

We remark that ED algorithms run into problems when the time between events gets too small. In dense systems with strong dissipation the time between events may tend towards zero. As a consequence the so-called "inelastic collapse" can occur, i.e. the divergence of the number of events per unit time. The problem of the inelastic collapse [MaBe94, NaYo94], can be handled using restitution coefficients dependent on the time elapsed since the last event [LuNa98]. If the time between a collision and the preceding one for at least one particle is smaller than a critical value $t_{c}$, we set $e_{n}=1$ and $e_{t}=-1$, i.e. the elastic values. The time $t_{\mathrm{c}}$ can be identified as a typical duration of a contact. We hope that the effect of $t_{\mathrm{c}}$ on the simulation results is negligible for small $t_{\mathrm{c}}$.

The codes for the ED algorithm of Lubachevsky used in this work were written 
by Timo Aspelmeier. The routines for the collision rules and the search for collisions by the author himself.

\subsubsection{Hard needles}

For this algorithm collision times for each pair of needles have to be determined numerically. We follow the algorithm proposed by Frenkel and Maguire [FrMa83]. Since the numerical procedure for finding collision events is time consuming, one first rejects all pairs if needles in the search for collisions which would not collide if they were spheres and then if they were discs, since we have angular momentum conservation and the needles rotate around their moving center of mass in a disc with constant normal. These two procedures - to consider an imaginary surrounding sphere and disc - supply also a lower and upper bound for the collision time. Inside this interval we have to find now the smallest root $^{3}$, corresponding to the next collision. This is done by a Newton procedure of second order. If a root is found a further lower root is searched in the interval of the previously found lower bound and the just found root, as long as no more lower roots can be found.

The whole algorithm is reasonably fast as long as there are only few needles in each cell of the linked-cell structure so that the time-consuming search for collisions is restricted to needles in the own and the neighboring cells. On the other hand we have to choose the linear dimension of these cells to be larger than the length of a needle, so that for high densities there are many needles in each cell and the algorithm becomes slow.

Since needles in 3 dimensions have zero excluded volume, all static properties of the elastic system are equal to those of an ideal gas. This has the advantage that the initial preparation of the system is trivial. We do not have to equilibrate the system, but can choose positions randomly distributed over the simulation box and orientations distributed isotropically. The velocities and angular velocities are taken from a Boltzmann distribution.

\subsubsection{Hard spheres}

The search for collisions in this case is much simpler than in the case of needles, since one only has to solve a quadratic equation. To get a well-defined initial configuration every simulation is first equilibrated with $e_{n}=1$ and $e_{t}=-1$ until the velocity distribution is Maxwellian and the spatial correlations are those of a classical gas of hard spheres. Then the restitution coefficients are set to the selected values.

\footnotetext{
${ }^{3}$ The conditions of contact are described in section 2.1.2. We have to find roots of the function $\boldsymbol{r}_{12} \boldsymbol{u}^{\perp}$ and test if $\left|s_{12}\right|<L / 2$ and $\left|s_{21}\right|<L / 2$.
} 


\subsection{Summary}

Two models are discussed where rotational degrees of freedom are important. We have chosen rough spheres and needles and focus on the simplest collision rules, which allow for a transfer of translational energy to rotational degrees of freedom. For spheres this is achieved by tangential restitution (in addition to normal restitution), for needles normal restitution is sufficient. We show that the time evolution can be formulated in terms of a pseudo-Liouville operator, thereby generalizing previous works from elastic collisions to inelastic ones. The presented formalism is general enough to include more realistic collision rules, for example Coulomb friction for small angles of impact and tangential restitution for large angles. The time evolution of the distribution function is formulated in terms of the corresponding time evolution operator, which we determine explicitly for the case of rough spheres. In the last part of this chapter we have discussed the event-driven simulation technique and describe the tricks to achieve a reasonably fast algorithm also for needles. 


\section{Homogeneous cooling state}

The aim of this chapter is to describe the time evolution of a gas of freely cooling smooth or rough spheres and needles which is dominated by two particle collisions, as discussed in the previous section.

In this context systems of smooth spheres or discs have been investigated by means of kinetic theory and computer simulations by several groups [GoZa93, GoZa93b, Na93, NaYo96, NoEr97, NoBr98, BrMo96, BrMo98, Deba97]. Most of the theories focus on latest times where interesting phenomena like formation of vortex patterns and clustering can be observed. For short times or not too high inelasticities the system remains homogeneous and the dynamics can be described by a decreasing average kinetic energy or granular temperature $T$ only. The assumption then is that scaling all velocities with the corresponding mean velocity, $v_{0}:=\sqrt{2 T / m}$, the shape of the velocity distribution function remains constant in time. This so-called homogeneous cooling state (HCS) is the starting point for a hydrodynamic analysis. Although many of the hydrodynamic theories use transport coefficients derived by the assumption of a Gaussian velocity distribution function, in general the shape is not Gaussian and deviations have been investigated in different contexts in refs. [GoZa93b, BrRu96, IcHa95, TaTa95, EsPo97].

Kinetic theory of rough, inelastic, circular disks was first discussed by Jenkins and Richman [JeRi85, JeRi85b]. These authors introduced two temperatures, one for the translational and one for the rotational degrees of freedom, and studied deviations from a two-temperature Maxwellian distribution using Grad's moment expansion. Subsequently Lun and Savage [LuSa87, Lu91] extended the approach to rough, inelastic spheres. A set of conservation equations and constitutive relations was derived from the Boltzmann equation assuming small inelasticity and surface roughness. Goldshtein and Shapiro [GoSh95] discuss in detail the homogeneous cooling state of rough spheres. They determine the asymptotic ratio of rotational to translational energy as a function of surface roughness and coefficient of normal restitution. They also assume a fixed shape of the velocity distribution function and assume that the dynamics can be described by a decreasing total kinetic energy. More recently, eventdriven simulations of rough spheres have been performed by McNamara and Luding [NaLu98].

In this chapter existing theories of the HCS are extended to the case where more than a single average kinetic energy is necessary to describe the time dependence of 
velocity distribution function. This will become clear when one considers a system of rough spheres which is initially prepared in an equilibrium ensemble. Then all degrees of freedom are equipartitioned and the translational and rotational velocities are distributed by a Maxwellian determined by a single temperature. Switching on restitution, the average rotational energy will in general decay with a different rate than the rotational energy. This means, even if one assumes that the shape of the distribution function is Gaussian, the Gaussian distributions for the two energies will have different widths or temperatures, which are now time dependent. Similarly a system of smooth spheres might initially be prepared in a pure Gaussian state. Switching on dissipation one may observe deviations from the Gaussian which can be described by the time dependence of cumulants of the distribution function. At the beginning higher than second order cumulants vanish but are not zero for later times, so that the full dynamics is not only described by the average kinetic energy, i.e. the second moment, but also by all higher moments. Since many theories are based on the assumption of a Gaussian distribution function, we will investigate if these cumulants remain small in time and check the range of validity of the Gaussian approximation.

Of particular interest will be the question on which time scales the dynamics proceeds. Since quantities like the average kinetic energy only change in collisions, we will introduce a "collisional time", which simply counts the number of collisions which have occurred until time $t$ [NoEr98]. We will see that on this time scale the corresponding equations will look quite "natural", but then one has to determine relations between "real" and "collisional" time. This is in general not possible, because the collision frequency changes in a complicated way with the cooling of the system. Furthermore we will see that the distribution function approaches an asymptotic fixed shape exponentially fast on the time scale of collisions ${ }^{1}$. Then the whole dynamics is described by a single decreasing kinetic energy, and therefore the assumption of a fixed shape of previous theories can be justified.

${ }^{1}$ The fixed shaped is characterized by a constant ratio of rotational and translational energy and (if one scales all velocities with the square root of the mean kinetic energy) by constant values of the cumulants. 


\subsection{Gaussian distribution}

In this section we will restrict ourselves to the assumption of a Gaussian velocity distribution function, but we will allow that in general the width or temperature of the Gaussian for the rotational degrees of freedom differs from the temperature of the translational ones. Here temperature defines the average kinetic energy per degree of freedom. We will see that the rotational energy decays with a different rate than the translational one so that introducing two different temperatures is mandatory. In the next section, we shall discuss non-Gaussian distributions and shall compute corrections perturbatively.

The average kinetic translational (rotational) energy and temperature at time $t$ are given by

$$
\begin{aligned}
\left\langle E_{\mathrm{tr}}\right\rangle(t) & =\frac{m}{2 N} \sum_{i} \int d \Gamma \rho(\Gamma ; t) \boldsymbol{v}_{i}^{2}=: \frac{D_{\mathrm{tr}}}{2} T_{\mathrm{tr}}(t), \\
\left\langle E_{\mathrm{rot}}\right\rangle(t) & =\frac{I}{2 N} \sum_{i} \int d \Gamma \rho(\Gamma ; t) \boldsymbol{\omega}_{i}^{2}=: \frac{D_{\mathrm{rot}}}{2} T_{\mathrm{rot}}(t) .
\end{aligned}
$$

Here $D_{\text {tr }}$ and $D_{\text {rot }}$ denote the total number of translational and rotational degrees of freedom respectively. We remind the reader that in this case the term temperature denotes the average (and time-dependent) kinetic energy per degree of freedom and is not a temperature in the sense of equilibrium statistical mechanics. See also footnote 2 in the introduction.

It is impossible to compute the above expectation values exactly and we have to resort to several approximations:

1. We assume that the $N$-particle probability distribution $\rho(\Gamma, t)$ factorizes in spatial and velocity variables

$$
\rho_{\mathrm{HCS}}(\Gamma ; t) \sim W\left(\boldsymbol{r}_{1}, \ldots, \boldsymbol{r}_{N}\right) \tilde{\rho}\left(\left\{\boldsymbol{v}_{i}, \boldsymbol{\omega}_{i}\right\}, t\right) .
$$

Further we assume that the function $W\left(\boldsymbol{r}_{1}, \ldots, \boldsymbol{r}_{N}\right)$ is homogeneous in space and only reflects that overlap configurations are forbidden. It gives zero weight to overlapping configurations and 1 otherwise. Needles have vanishing volume in configuration space, so that $W \equiv 1$.

2. We now demand that $\tilde{\rho}(\Gamma, t)$ depends on time only via the average kinetic energy of translation and rotation:

$$
\tilde{\rho}\left(\left\{\boldsymbol{v}_{i}, \boldsymbol{\omega}_{i}\right\}, t\right) \propto \tilde{\tilde{\rho}}\left(\left\{\boldsymbol{v}_{i}, \boldsymbol{\omega}_{i}\right\} ; T_{\mathrm{tr}}(t), T_{\mathrm{rot}}(t)\right) .
$$

3. In the last step we assume that $\tilde{\tilde{\rho}}$ factorizes in all particles and is Gaussian in all its momentum variables

$$
\tilde{\tilde{\rho}}\left(\left\{\boldsymbol{v}_{i}, \boldsymbol{\omega}_{i}\right\} ; T_{\mathrm{tr}}(t), T_{\mathrm{rot}}(t)\right) \propto \exp \left[-N\left(\frac{E_{\mathrm{tr}}}{T_{\mathrm{tr}}(t)}+\frac{E_{\mathrm{rot}}}{T_{\mathrm{rot}}(t)}\right)\right] .
$$


To determine the time dependence of $T_{\mathrm{tr}}(t)$ and $T_{\text {rot }}(t)$ we take time derivatives of eqs. (3.1) and use the identity eq. (2.41).

Then $\rho(\Gamma, t)$ is replaced by $\rho_{\mathrm{HCS}}(\Gamma ; t)$, resulting in

$$
\begin{aligned}
\frac{d}{d t} T_{\mathrm{tr}}(t) & =\frac{2}{D_{\mathrm{tr}}} \int d \Gamma \rho_{\mathrm{HCS}}(\Gamma ; t) i \mathcal{L} E_{\mathrm{tr}}=\frac{2}{D_{\mathrm{tr}}}\left\langle i \mathcal{L} E_{\mathrm{tr}}\right\rangle_{\mathrm{HCS}} \quad \text { and } \\
\frac{d}{d t} T_{\mathrm{rot}}(t) & =\frac{2}{D_{\mathrm{rot}}} \int d \Gamma \rho_{\mathrm{HCS}}(\Gamma ; t) i \mathcal{L} E_{\mathrm{rot}}=\frac{2}{D_{\mathrm{rot}}}\left\langle i \mathcal{L} E_{\mathrm{rot}}\right\rangle_{\mathrm{HCS}} .
\end{aligned}
$$

All that remains to be done are high-dimensional phase-space integrals, the details of which are delegated to appendices A.1.1 and A.2, for spheres and needles.

\subsubsection{Results for spheres}

After integration over phase space has been performed (see appendix A.1.1 for details), we find

$$
\begin{aligned}
& \frac{D_{\mathrm{tr}}}{2} \frac{d}{d t} T_{\mathrm{tr}}(t)=\left\langle i \mathcal{L} E_{\mathrm{tr}}\right\rangle_{\mathrm{HCS}}=-G A T_{\mathrm{tr}}^{3 / 2}+G B T_{\mathrm{tr}}^{1 / 2} T_{\mathrm{rot}}, \\
& \frac{D_{\mathrm{rot}}}{2} \frac{d}{d t} T_{\mathrm{rot}}(t)=\left\langle i \mathcal{L} E_{\mathrm{rot}}\right\rangle_{\mathrm{HCS}}=G B T_{\mathrm{tr}}^{3 / 2}-G C T_{\mathrm{tr}}^{1 / 2} T_{\mathrm{rot}},
\end{aligned}
$$

with positive constants $A, B, C$, and $G$ depending on space dimensionality $D$. In two dimensions the constants in eqs. (3.7) are given by

$$
\begin{aligned}
G & =4 d \frac{N}{V} \sqrt{\frac{\pi}{m}} g(d), & A & =\frac{1-e_{n}^{2}}{4}+\frac{\eta_{t}}{2}\left(1-\eta_{t}\right), \\
B & =\frac{\eta_{t}^{2}}{2 q}, & C & =\frac{\eta_{t}}{2 q}\left(1-\frac{\eta_{t}}{q}\right) .
\end{aligned}
$$

and in three dimensions

$$
\begin{aligned}
G & =8 d^{2} \frac{N}{V} \sqrt{\frac{\pi}{m}} g(d), & A & =\frac{1-e_{n}^{2}}{4}+\eta_{t}\left(1-\eta_{t}\right), \\
B & =\frac{\eta_{t}^{2}}{q}, & C & =\frac{\eta_{t}}{q}\left(1-\frac{\eta_{t}}{q}\right) .
\end{aligned}
$$

$\eta_{t}$ is defined in eq. (2.29) and the pair correlation function at contact, $g(d)$, is defined in the usual way [Ha86] by:

$$
g\left(\left|\boldsymbol{R}_{1}-\boldsymbol{R}_{2}\right|\right):=\frac{V^{2}}{N^{2}} \sum_{i \neq j}\left\langle\delta\left(\boldsymbol{R}_{1}-\boldsymbol{r}_{i}\right) \delta\left(\boldsymbol{R}_{2}-\boldsymbol{r}_{j}\right)\right\rangle .
$$

For details see also appendix A.1.1. 
The Enskog value [ChCo60, NoEr98] of the collision frequency $\omega_{E}$, i.e. the average number of collisions which a particle suffers per unit time in $D$ dimensions is given by

$$
\omega_{E}:=S_{D} \frac{N}{V} g(d) d^{D-1} \sqrt{\frac{T_{\mathrm{tr}}(t)}{\pi m}} .
$$

$S_{D}$ is the surface of a unit sphere in $D$ dimensions. Note that always $\omega_{E} \propto G T^{1 / 2}$. We define the dimensionless time $\tau$ by

$$
d \tau=\omega_{E}\left(T_{\mathrm{tr}}\right) d t
$$

so that $\tau$ counts the collisions that on average each particle has suffered until time $t$. In a simulation this would be simply done by counting the number of collisions. The functional dependence of the two temperatures on $\tau$ is determined by

$$
\begin{aligned}
\frac{d}{d \tau} T_{\mathrm{tr}} & =-a T_{\mathrm{tr}}+b T_{\text {rot }}, \\
\frac{d}{d \tau} T_{\text {rot }} & =b T_{\mathrm{tr}}-c T_{\text {rot }}
\end{aligned}
$$

with properly defined $a, b, c$. Eq. (3.13) has a simple interpretation. In a given short interval $\Delta t$ a number of $\Delta \tau$ collisions occur. Due to these collisions translational energy decreases by an amount given by the first term, but there is also a gain term, reflecting that rotational energy is transfered to translational energy. Eq. (3.14) can be interpreted in a similar way. The solution of eqs. $(3.13,3.14)$ can be written as

$$
\begin{aligned}
T_{\mathrm{tr}} & =c_{1} K_{+} \exp \left(-\lambda_{+} \tau\right)+c_{2} K_{-} \exp \left(-\lambda_{-} \tau\right), \\
T_{\text {rot }} & =c_{1} \exp \left(-\lambda_{+} \tau\right)+c_{2} \exp \left(-\lambda_{-} \tau\right), \\
K_{ \pm} & =\frac{1}{2 b}\left(c-a \pm \sqrt{(c-a)^{2}+4 b^{2}}\right) \\
\lambda_{ \pm} & =\frac{1}{2}\left(c+a \mp \sqrt{(c-a)^{2}+4 b^{2}}\right) .
\end{aligned}
$$

The constants $c_{1}$ and $c_{2}$ are determined by the initial conditions. It holds for all $e_{t}$, $e_{n}$, and $q$ that $\lambda_{-}>0, \lambda_{+}>0$ and $\lambda_{-}>\lambda_{+}$. Therefore for long times the ratio of $T_{\text {tr }} / T_{\text {rot }}$ is determined by $K_{+}$. Integrating equation (3.12) and inserting eq. (3.15) provides a relation between $t$ and $\tau$ :

$$
S_{D} \frac{N}{V} g(d) d^{D-1} \sqrt{\frac{1}{\pi m}}\left(t-t_{0}\right)=\int_{\tau_{0}}^{\tau}\left(c_{1} K_{+} \exp \left(-\lambda_{+} \tilde{\tau}\right)+c_{2} K_{-} \exp \left(-\lambda_{-} \tilde{\tau}\right)\right)^{-1 / 2} d \tilde{\tau} .
$$

The solution of the remaining integral ${ }^{2}$ gives no further insight.

\footnotetext{
${ }^{2}$ The remaining integral is of the form $\int_{0}^{x_{1}}\left(e^{\left(-\lambda_{1} x\right)}+b e^{\left(-\lambda_{2} x\right)}\right)^{-1 / 2} d x$ and can be calculated by a
} 


\section{Asymptotic solution}

We now assume that the ratio $T_{\text {tr }} / T_{\text {rot }}$ has reached its asymptotic value $K_{+}$for some $\tau>\tau_{0}$ or equivalently $t>t_{0}$ and substitute $T_{\text {rot }}=T_{\text {tr }} / K_{+}$into eq. (3.8). We obtain

$$
\frac{d}{d t} T_{\mathrm{tr}}=-F T^{3 / 2}
$$

The resulting equation is of the same functional form as for homogeneous cooling of smooth spheres, except for the coefficient $F$, which contains all the dependence on system parameters. Its solution is given by

$$
T_{\mathrm{tr}}=\frac{T_{\mathrm{tr}}\left(t_{0}\right)}{\left[1+T_{\mathrm{tr}}\left(t_{0}\right)^{1 / 2}(F / 2)\left(t-t_{0}\right)\right]^{2}} \sim \frac{1}{[(F / 2)(t)]^{2}},
$$

i.e. Haff's [Ha83] law of homogeneous cooling. We have determined two time scales, first an exponentially fast decay (measuring time in collisions) towards a state where we find a constant ratio of translational and rotational energy. As long as dissipation is small, we can approximate the Enskog-collision frequency for sufficiently short times by its initial value $\omega(t) \sim \omega(0)$ so that we find exponential behavior also in real time. The second stage of relaxation is characterized by a slow, algebraic decay of both energies, such that their ratio remains constant.

To get the full time dependence we solve eq. (3.7) numerically ${ }^{3}$. The two time regimes are clearly seen for initial conditions $T_{\mathrm{tr}}(0)=1$ and $T_{\text {rot }}(0)=0$, i.e. a system prepared in an equilibrium state of perfectly smooth spheres. We show in fig. 3.1 in a double logarithmic plot the time dependence of the total energy $E=\frac{3}{2}\left(T_{\mathrm{tr}}(t)+T_{\text {rot }}(t)\right)$ and the ratio $T_{\mathrm{tr}}(t) / T_{\mathrm{rot}}(t)$. Time is plotted in units of $\frac{2}{3} G T_{\mathrm{tr}}^{1 / 2}(0)$. We have chosen $e_{n}=0.9$ and $e_{t}=-0.8$. In addition we plot data of a simulation of 1000 particles in a box of length $16 d$. For details of the simulations see sections 2.4 and 4.2.

\section{Dependence on $e_{n}$ and $e_{t}$}

We now discuss the dependence of free cooling on $e_{n}$ and $e_{t}$. Of particular interest is the long-time decay of translational and rotational energy as a function of $e_{n}$ and $e_{t}$. We would expect the decay to be slowest if energy is lost only due to normal

series expansion of the integrand for $|b|<1$ and $\lambda_{2}>\lambda_{1}$ :

$$
\begin{aligned}
\int_{0}^{x_{1}} \frac{1}{\sqrt{e^{\left(-\lambda_{1} x\right)}+b e^{\left(-\lambda_{2} x\right)}}} d x & = \\
& \frac{2}{\sqrt{\pi}} \sum_{n=0}^{\infty}\left(\frac{\Gamma\left(n+\frac{1}{2}\right)(-b)^{n}\left(1-e^{\left(-1 / 2 x_{1}\left(-\lambda_{1}+2 n \lambda_{2}-2 n \lambda_{1}\right)\right)}\right)}{\Gamma(n+1)\left(-\lambda_{1}+2 n \lambda_{2}-2 n \lambda_{1}\right)}\right)
\end{aligned}
$$

${ }^{3}$ The numerical integration scheme we use is the procedure stiff from [PrTe94]. 


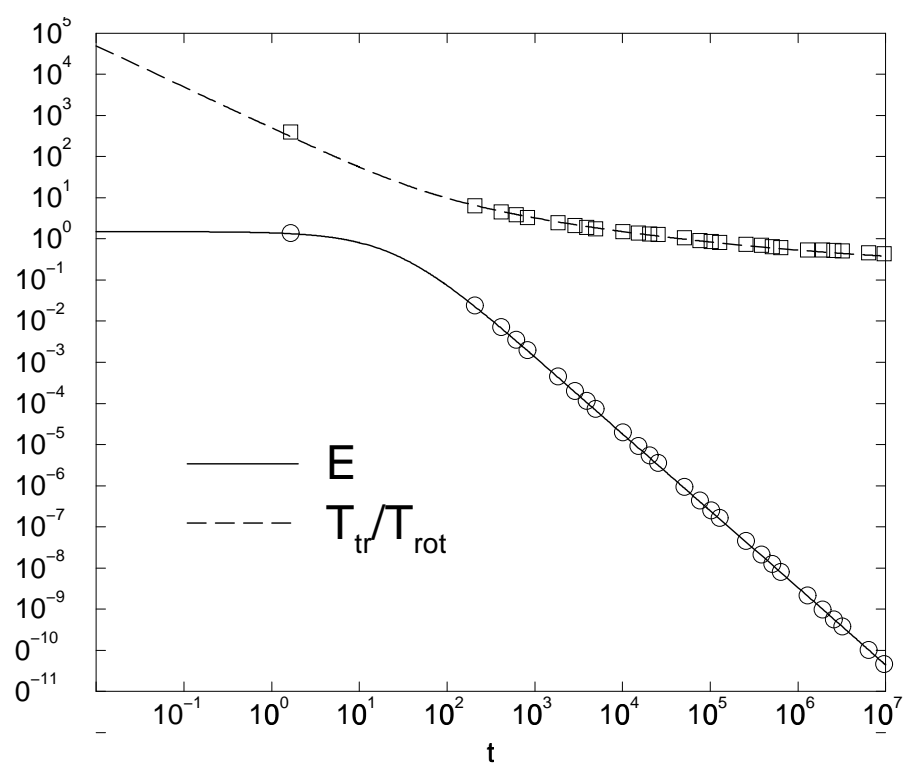

Figure 3.1.: Theoretical prediction (lines) for spheres for the total energy $E=$ $\frac{3}{2}\left(T_{\mathrm{tr}}(t)+T_{\text {rot }}(t)\right)$ and the ratio $T_{\mathrm{tr}}(t) / T_{\text {rot }}(t)$ versus time. Time is plotted in units of $\frac{2}{3} G T_{\mathrm{tr}}^{1 / 2}(0)$. We have chosen $e_{n}=0.9$ and $e_{t}=-0.8$. The symbols represent data of a simulation of 1000 particles in a box of length $16 \mathrm{~d}$.

restitution and not due to roughness. Hence, as a function of $e_{t}$, translational and rotational energy should persist for the longest times for $e_{t}= \pm 1$. In between, i.e. $-1<e_{t}<1$, we expect a faster decay, because surface roughness is an additional mechanism for the dissipation of energy. These expectations are born out by the following detailed discussion of eq. (3.22). We restrict our discussion to the case of two dimensions, where $F$ is given by $F=G\left(A-B / K_{+}\right)$. Asymptotically, for $t \rightarrow \infty$, the translational energy decays like $T_{\mathrm{tr}} \approx\left(\frac{F}{2} t\right)^{-2}$ and the rotational energy like $T_{\text {rot }} \approx\left(\frac{F \sqrt{K_{+}}}{2} t\right)^{-2}$. In a double-logarithmic plot of $T_{\mathrm{tr}}$ and $T_{\text {rot }}$ against $t$ one should observe straight lines with slope -2 and axial sections (at $t=1$ ) given by $-2 \ln (F / 2)$ for the translational energy, and by $-2 \ln \left(F \sqrt{K_{+}} / 2\right)$ for the rotational energy. For the same slope a larger axial section implies persistence for longer times, i.e. a 'slower' decay in time. In fig. 3.2 we plot the prefactors $F$ and $F \sqrt{K_{+}}$against $e_{t}$ for $e_{n}=0.99$.

As a function of $e_{t}, F$ is smallest for $e_{t} \rightarrow \pm 1$ corresponding to the cases where no energy is lost due to friction. The decay of translational energy is then pushed out to the longest time scales. In fig. 3.2, the maximum of $F$ is reached for $e_{t_{\mathrm{tr}}}^{\max } \approx 0.17$, corresponding to the 'fastest' decay of $T$. For $e_{t}=-1$ we find that $F \sqrt{K_{+}}=0$, because the rotational energy remains constant as a function of time. $F \sqrt{K_{+}}$reaches 


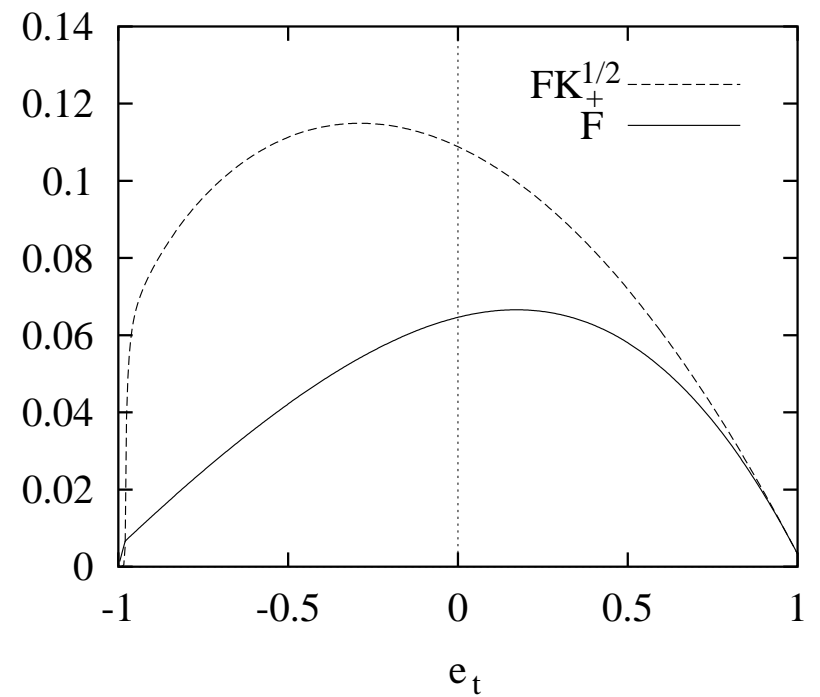

Figure 3.2.: Dependence of the asymptotic decay-prefactors $F$ and $F \sqrt{K_{+}}$on $e_{t}$ for $e_{n}=0.99$.

its maximum for $e_{t_{\mathrm{rot}}}^{\max } \approx-0.29 \neq e_{t_{\mathrm{tr}}}^{\max }$, so that the decay of rotational energy is 'fastest' for a value of $e_{t}$, different from the one where the translational energy decays fastest. In the limit $e_{t} \rightarrow 1$ the axial sections for $T$ and $R$ have approximately the same values. In this case the ratio $K_{+}$is close to unity, reflecting the rather effective exchange of rotational and translational energy for rough spheres and thus approximate equipartition.

\section{Comparison with simulations}

In this section we compare our theoretical analysis with results of simulations of three-dimensional systems performed by Luding and McNamara [LuHu98]. We introduce the dimensionless temperatures $T=T_{\mathrm{tr}} / T_{\mathrm{tr}}(0)$ and $R=T_{\mathrm{rot}} / T_{\mathrm{tr}}(0)$ and a dimensionless time

$$
\tilde{t}=\frac{2}{3} G t
$$

and solve eq. (3.7) analytically for short times and initial conditions $T(0)=1$ and $R(0)=0$. We get $T=1-A \tilde{t}+\mathcal{O}\left(\tilde{t}^{2}\right)$ and $R=B \tilde{t}+\mathcal{O}\left(\tilde{t}^{2}\right)$. Hence, rescaling time $\tilde{t} \rightarrow A \tilde{t}$ and rotational temperature $R \rightarrow R A / B$ all data can be collapsed for short times. This allows us to investigate the dependence of cooling on system parameters like $e_{n}, e_{t}$ and density in a clear way.

Various systems characterized by volume fraction $\rho=\frac{4 \pi}{3} d^{3} \frac{N}{V}$ and particle number $N$ have been simulated. One system with density $\rho=0.087$ and $N=1331$ particles 
is denoted as medium; two other systems with lower density have the parameters $\rho=0.0021, N=4096$ (denoted by dilute), and $\rho=0.0023, N=68921$ (denoted by large). The abbreviation corresponds to the density, only for the large system one should read "dilute and large". Finally, a system with higher density, i.e. $\rho=0.23$ and $N=54872$, denoted by dense, is examined. To calculate the pair correlation function at contact, we use the Carnahan-Starling formula in 3D [Ha86]:

$$
4 \rho g(2 a)=\frac{1+\rho+\rho^{2}-\rho^{3}}{(1-\rho)^{3}}-1=4 \rho \frac{1-\rho / 2}{(1-\rho)^{3}},
$$

Initially, the normalized energies are $T=1$ and $R=0$ for all data presented here.

In fig. 3.3 we plot $T$ against normalized time $A \tilde{t}$ for $e_{n}=0.99$ and various values of the tangential restitution $e_{t}$. We observe reasonable agreement between theory and simulation over many orders of magnitude in time. For $e_{t}<0.5$ most of the dependence on $e_{t}$ is taken into account by our scaling, $\tilde{t} \rightarrow A \tilde{t}$, so that the scaled data almost collapse for $e_{t}<0.5$.

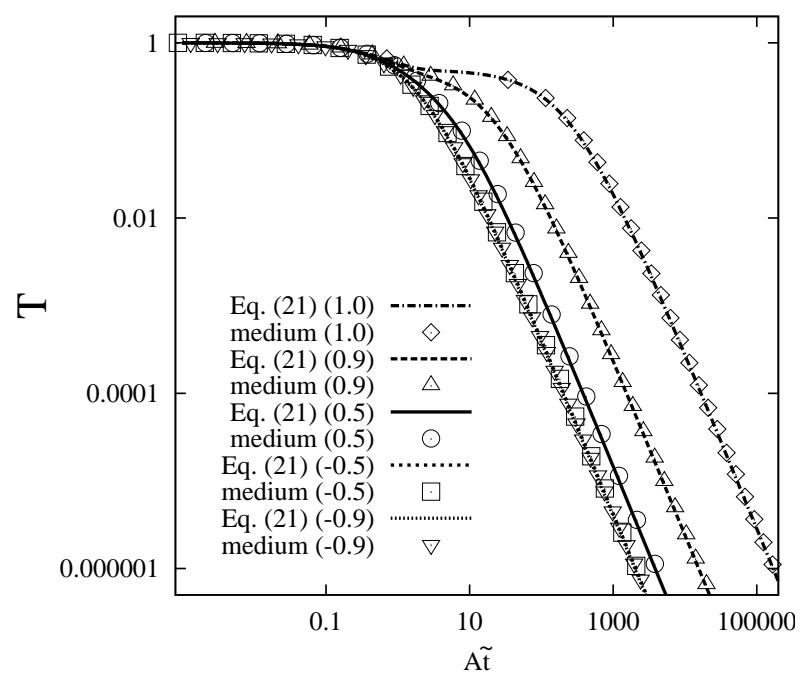

Figure 3.3.: $T$ as function of rescaled time $A \tilde{t}$ in $3 \mathrm{D}$. The symbols correspond to simulations with $N=1331, \rho=0.087$ (medium), $e_{n}=0.99$, and different $e_{t}$ as given in brackets in the inset. The curves represent numerical solutions of eqs. (3.7) with the three-dimensional constants from eqs. (3.9).

In fig. 3.4 we compare simulations of different systems with the numerical solution of eqs. 3.7. Only the dense simulations deviate from the theoretical result.

In fig. 3.5 we plot $R A / B$ versus normalized time $A \tilde{t}$ for medium and dense systems. We find that $R A / B$ increases proportional to $A \tilde{t}$ for small times $(A \tilde{t}<0.1)$, reflecting 


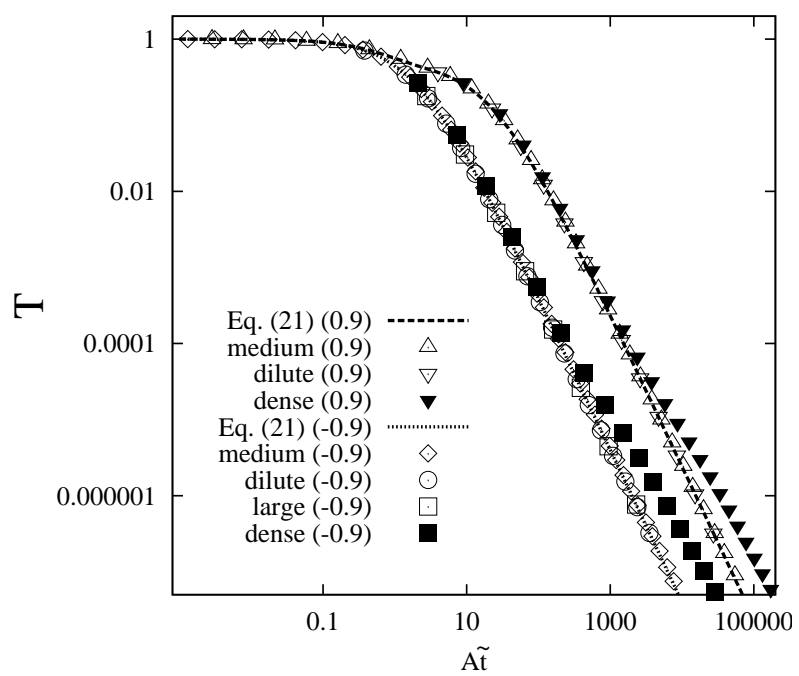

Figure 3.4.: $T$ as function of rescaled time $A \tilde{t}$ in $3 \mathrm{D}$ from simulations with $e_{n}=0.99$, and $e_{t}=-0.9$ or $e_{t}=+0.9$ as given in brackets. Different symbols correspond to simulations with $N=1331, \rho=0.087$ (medium), $N=$ 4096, $\rho=0.0021$ (dilute), $N=68921, \rho=0.0023$ (large), and $N=$ $54872, \rho=0.23$ (dense). The curves are numerical solutions of eqs. (3.7) with the three-dimensional constants from eqs. (3.9).

the activation of initially 'cold' rotational degrees of freedom due to collisions. This feature as well as the full time dependence is well reproduced by our theoretical analysis.

\section{Almost smooth spheres}

In fig. 3.6 we present $T, R A / B$ and the ratio of $T$ and $R A / B$ as a function of scaled time $A \tilde{t}$ for $e_{n}=0.6$ and $e_{t}=-0.9$, where an interesting structure is observed. The symbols correspond to a system with $\rho=0.087, N=1331$. The data are in good agreement with the theoretical curves. The loss of energy during collisions is predominantly due to normal restitution and only after the translational energy has decayed to a very small value $\left(T<10^{-5}\right)$ does one observe the energy loss due to tangential restitution. The two regimes can be discussed analytically using eqs. (3.9). For intermediate times, when the translational energy is still appreciable, the equations can be simplified for almost smooth spheres, i.e. $\left(e_{t} \approx-1\right)$ :

$$
\begin{aligned}
& \frac{d}{d \tilde{t}} T=-A T^{3 / 2} \\
& \frac{d}{d \tilde{t}} R=-C T^{1 / 2} R .
\end{aligned}
$$




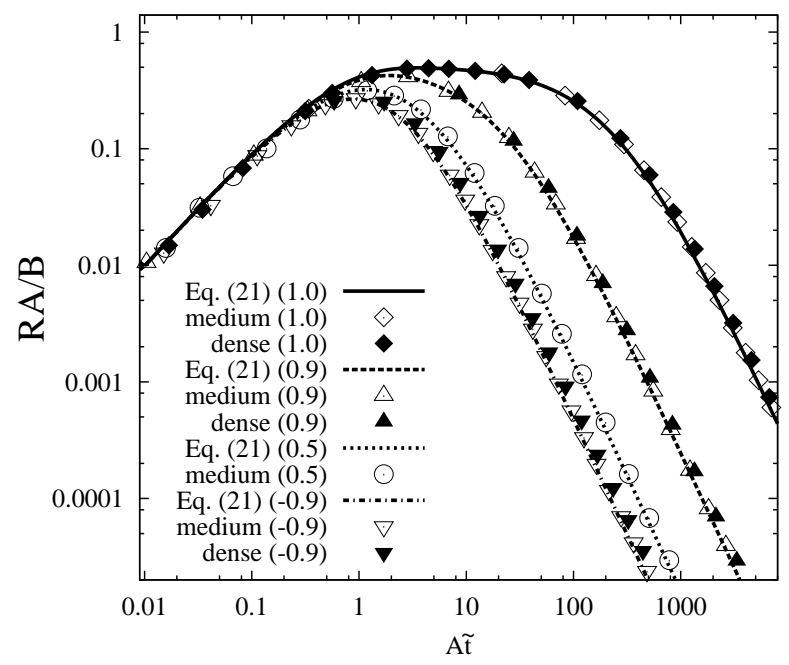

Figure 3.5.: $R A / B$ as function of rescaled time $A \tilde{t}$. The data are selected situations from fig. 3.3. The curves represent the numerical solutions of eqs. (3.7).

We have neglected terms of $\mathcal{O}\left(\left(1+e_{t}\right)^{2}\right)$ and approximate $A \approx\left(1-e_{n}^{2}\right) / 4$ and $C \approx 5\left(1+e_{t}\right) / 14$. The solution for $T$ is that of smooth spheres, decaying like $T(\tilde{t}) \approx(A \tilde{t} / 2)^{-2}$ for large $\tilde{t}$. Substituting this result into the equation for $R$, we find $R(\tilde{t}) / R\left(\tilde{t}_{0}\right)=\left(\tilde{t} / \tilde{t}_{0}\right)^{-\alpha}$ with $\alpha=2 C / A$. The exponent $\alpha$ depends continuously on $e_{n}$ and $e_{t}$. Here $\tilde{t}_{0}$ is some intermediate time scale, larger than the time for the initial increase of $R$, but smaller than the time scale to reach the true asymptotic state. The above algebraic decay is shown in fig. 3.6 as a straight dashed line with $\alpha \approx 0.396$. Once the translational energy has decayed to a very small value as compared to the rotational energy, all terms in the differential equations for $R$ and $T$ are equally important. We then observe a crossover from a $\tilde{t}^{-\alpha}$ to a $\tilde{t}^{-2}$ decay of the rotational energy. This is the true asymptotic state characterized by a constant ratio $T / R$ and has been discussed above. The crossover between the two regimes shows up as a parallel shift for $T$ [see figs. 3.6 and 3.7], because the translational energy decays like $\tilde{t}^{-2}$ in both regimes, but with a different prefactor.

When $e_{n}$ is increased to a value close to unity, i.e. the elastic case, the intermediate time regime disappears, because normal and tangential restitution are equally important. This is demonstrated in fig. 3.7, where we show $T$ plotted against the normalized time $A \tilde{t}$ for medium density $\rho=0.087, e_{t}=-0.9$ and different values of $e_{n}$, as given in the legend.

In the intermediate time regime all curves follow the decay of smooth spheres (see eq. (3.25)), which is independent of $e_{n}$, because we use scaled time $A \tilde{t}$. In the true asymptotic regime, all curves have the same slope however with an axial section, which increases with decreasing $e_{n}$. Without scaling $\tilde{t}$ with $A$ the axial 


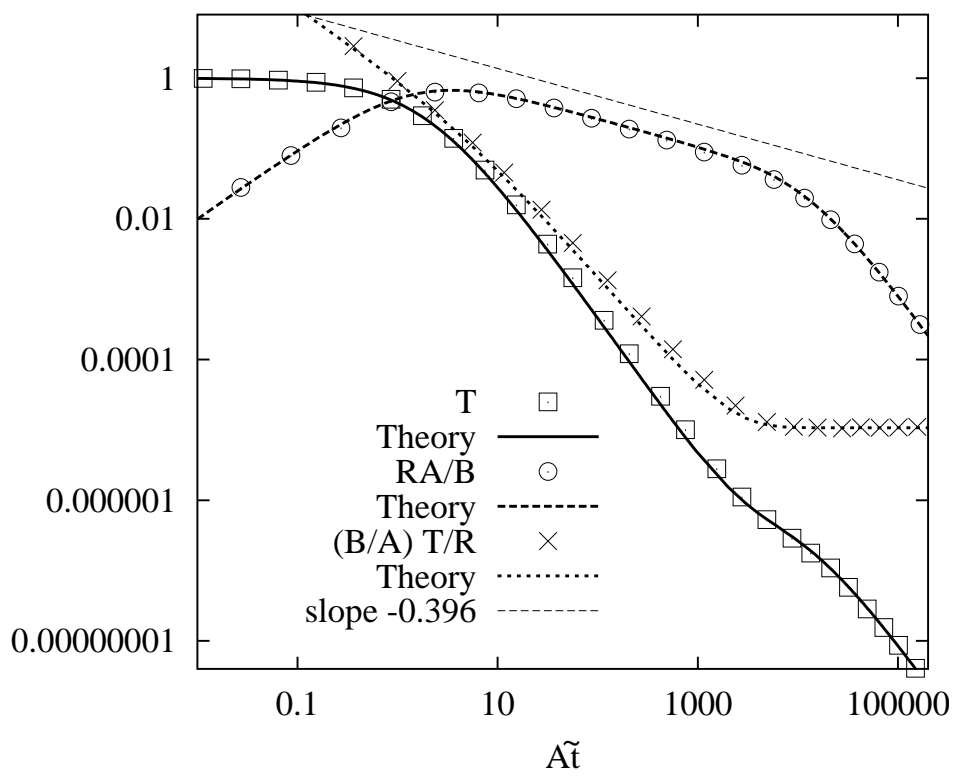

Figure 3.6.: $T, R A / B$, and $(B / A) T / R$ for $3 \mathrm{D}$ simulations with $e_{t}=-0.9$ and $e_{n}=$ 0.6 (symbols) as a function of scaled time $A \tilde{t}$. The lines give the solution of eqs. (3.9).

section decreases with decreasing $e_{n}$ reflecting the more efficient dissipation of energy for smaller $e_{n}$. The agreement between theory and simulations is quite good for values of $e_{n}$ as low as $e_{n}=0.6$, and even for $e_{n}=0.2$ only the crossover regime is not captured by theory.

\subsubsection{Results for needles}

In the case of needles we restrict ourselves to the case of perfectly smooth needles, i.e. $e_{t}=-1$. After some lengthy algebra, presented in appendix A.2, eq. (3.6) can be cast in the following form

$$
\begin{aligned}
& \frac{2 \dot{T}_{\mathrm{tr}}}{\gamma_{n} T_{\mathrm{tr}}^{3 / 2}\left(1+e_{n}\right)}=-\int_{\square} d^{2} r \frac{\left(1+\frac{T_{\mathrm{rot}}}{T_{\mathrm{tr}}} k r^{2}\right)^{1 / 2}}{1+k r^{2}} \\
& +\frac{1+e_{n}}{2} \int_{\square} d^{2} r \frac{\left(1+\frac{T_{\mathrm{rot}}}{T_{\mathrm{tr}}} k r^{2}\right)^{3 / 2}}{\left(1+k r^{2}\right)^{2}}, \\
& \frac{4 \dot{T}_{\mathrm{rot}}}{3 \gamma_{n} T_{\mathrm{tr}}^{3 / 2}\left(1+e_{n}\right)}=-\int_{\square} d^{2} r \frac{\frac{T_{\mathrm{rot}}}{T_{\mathrm{tr}}} k r^{2}\left(1+\frac{T_{\mathrm{rot}}}{T_{\mathrm{tr}}} k r^{2}\right)^{1 / 2}}{1+k r^{2}}
\end{aligned}
$$




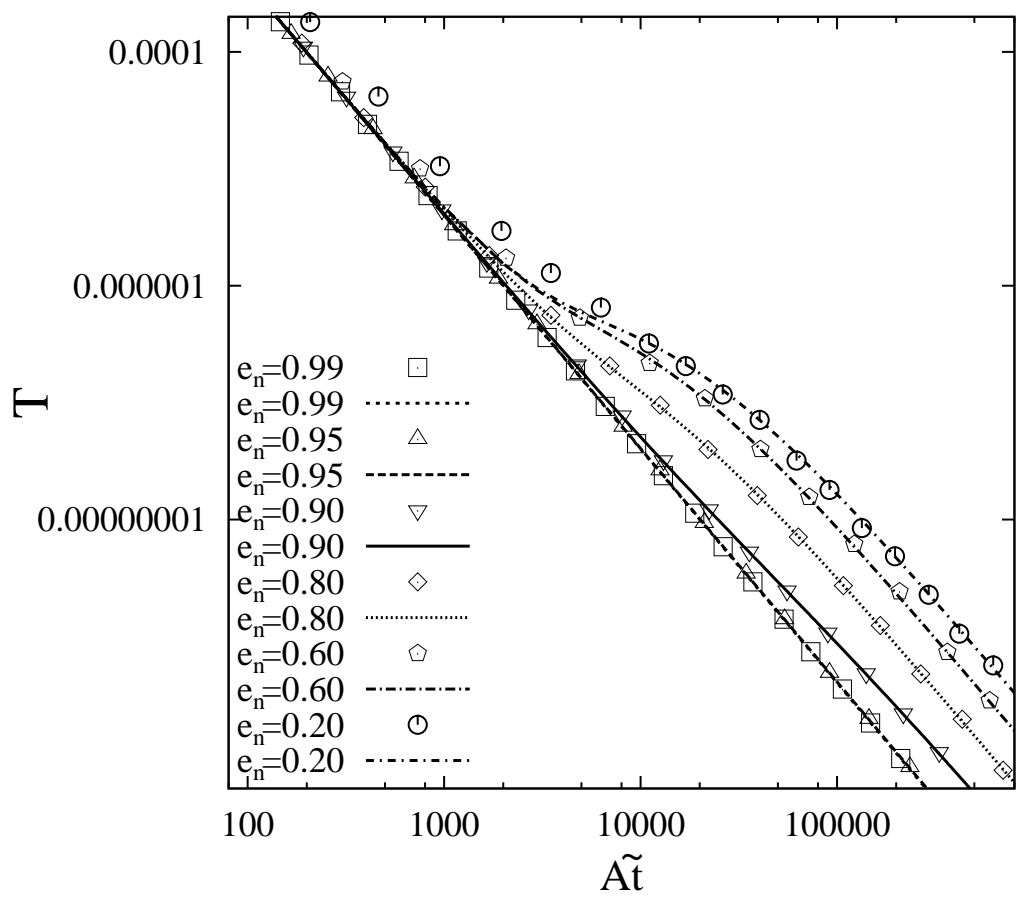

Figure 3.7.: $T$ as function of rescaled time $A \tilde{t}$. The density is $\rho=0.087, e_{t}=$ -0.9 and the restitution coefficient $e_{n}$ is given in the insert. The curves represent the numerical solutions of eqs. (3.9).

$$
+\frac{1+e_{n}}{2} \int_{\square} d^{2} r \frac{k r^{2}\left(1+\frac{T_{\mathrm{rot}}}{T_{\mathrm{tr}}} k r^{2}\right)^{3 / 2}}{\left(1+k r^{2}\right)^{2}}
$$

with $\gamma_{n}=\left(2 N L^{2} \sqrt{\pi}\right) /(3 V \sqrt{m})$ and $k=\left(m L^{2}\right) /(2 I)$. The two-dimensional integration extends over a square of unit length, centered at the origin.

In fig. 3.8 we plot the numerical solution ${ }^{4}$ of eqs. $(3.27,3.28)$ for $e_{n}=0.8$ and $k=6$ ( $k=6$ corresponds to a homogeneous mass distribution along the rod) as a function of time in units of $\gamma_{n} \sqrt{T_{\mathrm{tr}}(0)}$. In addition we have performed simulations of a system of 10000 needles, confined to a box of length $24 \mathrm{~L}$. (For details of the simulations see section 2.4 and 4.1.) We show the total kinetic energy $E=\frac{3}{2} T_{\mathrm{tr}}+T_{\text {rot }}$ (in units of $\left.T_{\mathrm{tr}}(\tau=0)\right)$ and the ratio $T_{\mathrm{tr}} / T_{\text {rot }}$. Analytical theory and simulation are found to agree within a few percent over eight orders of magnitude in time. $\left(T_{\text {rot }}(0)=0\right.$ has been chosen as initial condition). In this case we observe an even clearer separation

\footnotetext{
${ }^{4}$ For the numerical solution of eqs. $(3.27,3.28)$ the integrations have to be performed first. Therefore we use spherical coordinates, so that the integration over the angle can be performed analytically. The remaining integral is performed numerically by the procedure qromb of [PrTe94]. For the numerical integration of the differential equation we use odeint also taken from [PrTe94].
} 


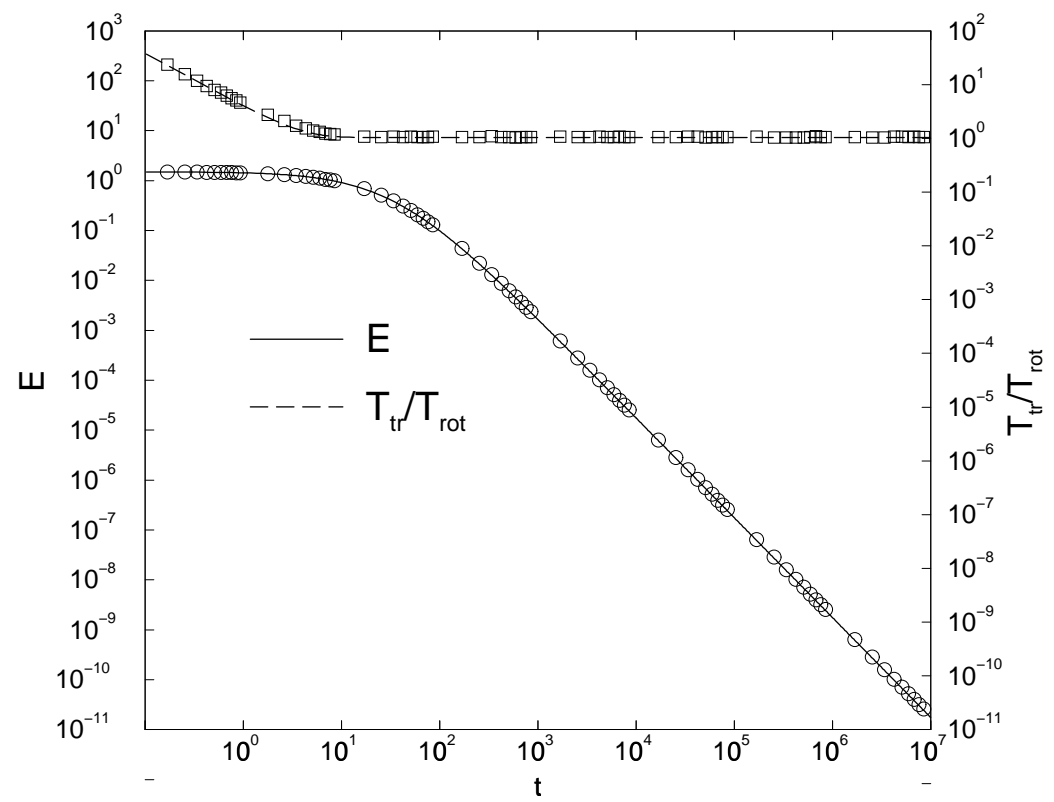

Figure 3.8.: Theoretical prediction (lines) for needles for the total energy $E=$ $\frac{3}{2}\left(T_{\mathrm{tr}}(t)+T_{\text {rot }}(t)\right)$ and the ratio $T_{\mathrm{tr}}(t) / T_{\text {rot }}(t)$ versus time. Time is plotted in units of $\gamma_{n} \sqrt{T_{\mathrm{tr}}(0)}$. The simulation data are from a system of 10000 needles in a box of length $24 L$ with $e_{n}=0.8$.

of time scales. The decay of $T_{\mathrm{tr}} / T_{\text {rot }}$ to a constant value $c$ happens on a time scale of order one. In this range of times the total kinetic energy $E$ remains approximately constant (on a logarithmic scale) and decays like $t^{-2}$ only after translational and rotational energy have reached a constant ratio.

\section{The constant ratio}

We plug the ansatz $c T_{\text {rot }}=T_{\text {tr }}$ into eqs. $(3.27,3.28)$ and recover Haff's law also for needles, i.e. $T_{\mathrm{tr}} \propto-T_{\mathrm{tr}}^{(3 / 2)}$ and $T_{\text {rot }} \propto-T_{\mathrm{rot}}^{(3 / 2)}$. To determine the constant $c$ we use $c \dot{T}_{\text {rot }}-\dot{T}_{\text {tr }}=0$, which yields an implicit equation for $c$, whose solution is plotted in fig. 3.9.

The asymptotic ratio $c$ depends on $k$ as well as on $e_{n}$. Interestingly we can choose $k$ in a way that equipartition holds for all $e_{n}$. To find that particular value of $k$ we set $c=1$ which yields an equation for $k$ :

$$
\left(1-e_{n}^{2}\right) \int d^{2} x \frac{1-\frac{3}{2} k^{*} r^{2}}{\sqrt{1+k^{*} r^{2}}}=0
$$

Equipartition holds for all values of $e_{n}<1$ if $k=\left(m L^{2}\right) /(2 I)$ is set to the particular value $\left(k^{*}=4.3607\right)$. For $e_{n}=1$, equipartition always holds, independent of $k$. 


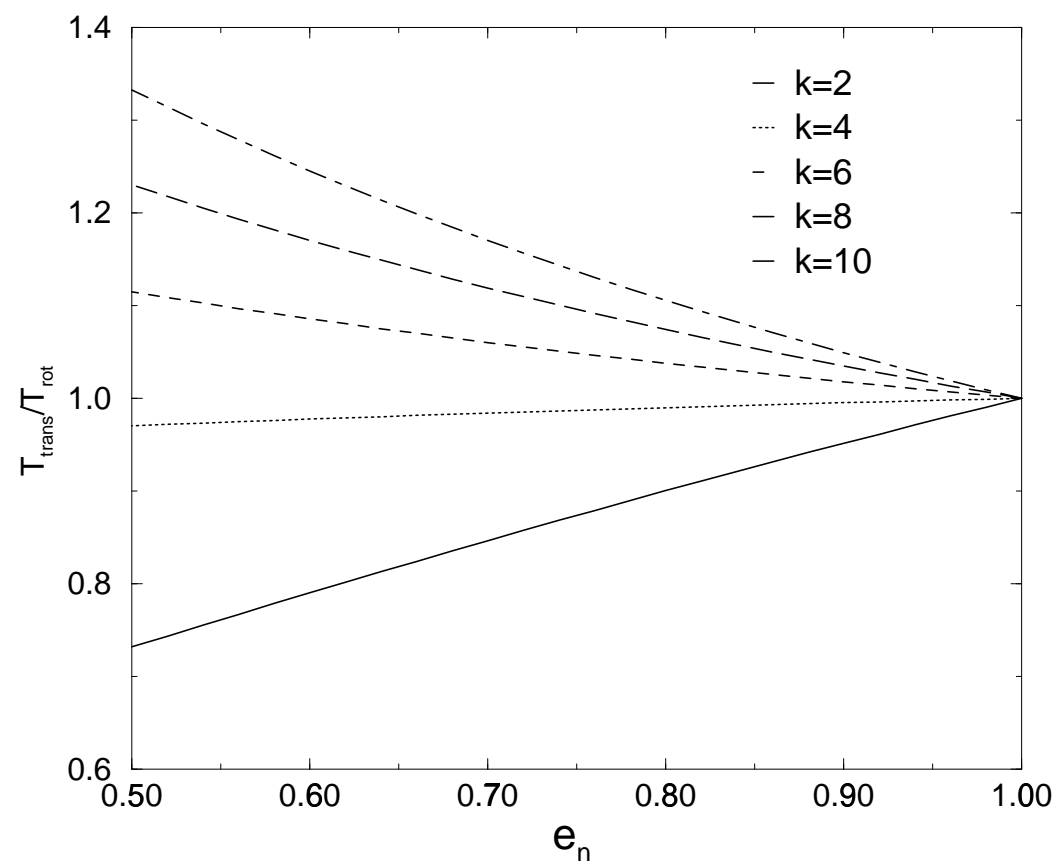

Figure 3.9.: Asymptotic ratio for $T_{\mathrm{tr}} / T_{\text {rot }}$ as a function of $e_{t}$ for different values of $k$.

For $k<k^{*}$ we find $T_{\mathrm{tr}}<T_{\text {rot }}$ and for $k>k^{*}, T_{\mathrm{tr}}>T_{\text {rot }}$. Hence the distribution of mass along the rods determines the asymptotic ratio of rotational and translational energy, including equipartition as a special case.

The asymptotic solution discussed above is approached for arbitrary initial conditions for long times. If a totally elastic system is prepared in an initial condition with $T_{\mathrm{tr}} \neq T_{\text {rot }}$ we expect that the equilibrium state (equipartition) is reached exponentially fast with relaxation rate given by $\nu \propto \gamma \sqrt{T_{\mathrm{tr}}}$. As long as energy dissipation due to inelastic collisions is small we expect a similar behavior, which is confirmed by the numerical solution of eqs. (3.27) and (3.28) and the simulations.

\subsubsection{Summary}

The homogeneous cooling state for rough spheres and needles is discussed. We formulate the $N$-particle distribution function, which we assume to be homogeneous in space and velocities distributed by a Gaussian with time-dependent translational and rotational temperatures, $T_{\operatorname{tr}}$ and $T_{\text {rot }}$. This leads to two coupled differential equations for the two temperatures. For sufficiently rough spheres and for needles, the relaxation of translational and rotational kinetic energy is characterized by two time 
scales: (1) An exponentially fast decay towards a state with constant ratio of translational to rotational energy and (2) an algebraically slow decay of the whole energy, such that the above ratio remains constant in time. The theoretically predicted cooling dynamics is confirmed by computer simulations of systems of small or moderate density, where no shearing or cluster instability is observed and the system remains homogeneous [LuHu98, HuAs99]. For almost smooth spheres, i.e. if the coefficients of normal and tangential restitution are such that the energy is lost mainly due to normal restitution, we observe an intermediate time regime which is characterized by an algebraic decay of translational and rotational energy: Translational energy decays according to Haff's law $t^{-2}$, whereas rotational energy decays with an exponent that depends continuously on $e_{n}$ and $e_{t}$.

\subsection{Non-Gaussian distribution}

Deviations from the Gaussian state are expressed by cumulants of higher order. We consider smooth and rough spheres. For smooth spheres we are able to determine the full dynamics of higher cumulants up to sixth order. For rough spheres we restrict ourselves to the state where the deviations from a Gaussian state have reached their asymptotic values. The collision integrals are calculated by means of computer algebra programs.

\subsubsection{Smooth spheres}

In this section we consider smooth disks in two dimensions. Calculations of collision integrals are much simpler due to a reduced number of degrees of freedom. This will allow us to test in this case the pertubative procedure to higher order than in the case of rough spheres.

The velocity distribution function is expanded around a Gaussian distribution in terms of generalized Laguerre or Sonine polynomials. The coefficients of this expansion are denoted with $a_{l}$. For time-independent $a_{l}$ this expansion has first been done by Goldshtein and Shapiro [GoSh95], who also determined the first nonvanishing coefficient $a_{2}$. Noije and Ernst [NoEr98b] also calculated $a_{2}$ and found a different result. In a more recent paper Brilliantov and Pöschel [BrPo99] consider the dynamics of $a_{2}$ up to order $a_{2}^{3}$ but they neglect higher order coefficients $a_{i}$ for $i>2$. We will investigate the influence of higher coefficients $a_{3}, \ldots, a_{6}$, which we assume to be time dependent.

We recapitulate the dynamics of smooth spheres with normal restitution, which is a special case of the more general rough sphere model. We consider a system of $N$ smooth, inelastically colliding spheres with radius $d$ confined to a $D$-dimensional volume $V$, so that the homogeneous density is given by $n:=\frac{N}{V}$. The position of each sphere is denoted by $\boldsymbol{r}_{i}$ and each particle has a velocity $\boldsymbol{v}_{i}$. The collision rules given 
in eq. (2.14) simplify to

$$
\begin{aligned}
& \boldsymbol{v}_{i}^{\prime}=\boldsymbol{v}_{i}-\frac{1+e_{n}}{2}\left(\boldsymbol{v}_{i j} \hat{\boldsymbol{r}}_{i j}\right) \hat{\boldsymbol{r}}_{i j}, \\
& \boldsymbol{v}_{j}^{\prime}=\boldsymbol{v}_{j}+\frac{1+e_{n}}{2}\left(\boldsymbol{v}_{i j} \hat{\boldsymbol{r}}_{i j}\right) \hat{\boldsymbol{r}}_{i j},
\end{aligned}
$$

where $\boldsymbol{v}_{i j}=\boldsymbol{v}_{i}-\boldsymbol{v}_{j}$ and $\hat{\boldsymbol{r}}_{i j}=\boldsymbol{r}_{i j} / r_{i j}$. Angular velocities are not changed so we do not consider them, which simplifies the calculations enormously. We are interested in the dynamics of a freely cooling system of granular particles, which we assume to remain homogeneous for all times. The system will initially be prepared in a Gaussian state, so that deviations from the Gaussian should be small for short times and small inelasticity. We assume that all particles are equally distributed and expand the one particle distribution function $\rho_{i}\left(\boldsymbol{v}_{i}, t\right)$ in generalized Laguerre polynomials ${ }^{5} L_{n}^{\alpha}$ around the Gaussian state.

An average velocity $v_{0}(t)$ can be defined via the second moment of the distribution function

$$
\int d \Gamma \rho \boldsymbol{v}_{1}^{2}=: \frac{D}{2} v_{0}^{2}(t)
$$

and the temperature $T$ is defined in terms of $v_{0}(t)$ by $T(t):=\frac{m}{2} v_{0}^{2}(t)$. All velocities are now scaled by $v_{0}(t)$ and denoted with $\boldsymbol{c}_{i}=\boldsymbol{v}_{i} / v_{0}(t)$. We expand the distribution function around a Gaussian function in the scaled variable $\boldsymbol{c}$ and time-dependent coefficients $a_{n}(t)$. The general ansatz for the $N$-particle distributions function for the homogeneous cooling then reads

$$
\begin{aligned}
\rho(\Gamma, t) \propto W\left(\boldsymbol{r}_{1}, \ldots, \boldsymbol{r}_{n}\right) & \prod_{i=1}^{N} \rho_{i}\left(\boldsymbol{v}_{i}, t\right), \text { and } \\
\rho_{i}\left(\boldsymbol{v}_{i}, t\right):= & \frac{1}{\left(v_{0}(t) \sqrt{\pi}\right)^{D}} \tilde{\rho}_{i}\left(\boldsymbol{c}_{i}, t\right):= \\
& \frac{1}{\left(v_{0}(t) \sqrt{\pi}\right)^{D}} \exp \left(-\boldsymbol{c}_{i}^{2}\right) \sum_{n=0}^{\infty} a_{n}(t) L_{n}^{\alpha}\left(\boldsymbol{c}_{i}^{2}\right)
\end{aligned}
$$

with $\alpha=D / 2-1$ in $D$ dimensions. If we choose $a_{0}=1$ then $\rho_{i}$ is properly normalized, so that $\int d \boldsymbol{v}_{\boldsymbol{i}} \rho_{i}=1$.

We express $\boldsymbol{v}_{i}^{2}$ by the first and second Laguerre polynomial eqs. (A.32)(A.33)

$$
\boldsymbol{v}_{i}^{2}=-L_{1}^{\alpha}\left(\boldsymbol{v}_{i}^{2}\right)-(\alpha+1) L_{0}^{\alpha}\left(\boldsymbol{v}_{i}^{2}\right)
$$

\footnotetext{
${ }^{5}$ For a definition of the Laguerre polynomials $L_{n}^{\alpha}$ and the choice of $\alpha$ see [MaOb66] and appendix A.3.
} 
and find using the orthogonality relations eq. (A.37)

$$
\int d \boldsymbol{v}_{i} \rho_{1}\left(\boldsymbol{v}_{i}, t\right) \boldsymbol{v}_{i}^{2}=v_{0}^{2}\left(\frac{D}{2}-\left(\begin{array}{c}
1+\alpha \\
1
\end{array}\right) a_{1}\right)
$$

which implies together with eq. (3.31) that $a_{1}=0$ for all times [GoSh95, NoEr98b, BrPo99]. See also appendix A.4.

We remark here that the coefficients $a_{i}(t)$ are given using the orthogonality relations of the Laguerre polynomials by

$$
a_{l}=\frac{1}{\left(\begin{array}{c}
l+\alpha \\
l
\end{array}\right)} \int d \Gamma \rho(\Gamma, t) L_{l}^{\alpha}\left(\left(\frac{\boldsymbol{v}_{1}}{v_{0}(t)}\right)^{2}\right) .
$$

We denoted the binomial coefficients by $\left(\begin{array}{l}a \\ b\end{array}\right)$.

\section{Dynamics of moments}

Using eqs. (3.31) and (A.15) the time dependence of $T(t)=\frac{m}{2} v_{0}^{2}$ is given by (see appendix A.4)

$$
\frac{d}{d t} T=\frac{d}{d t} \frac{m}{2} v_{0}^{2}=-2 \gamma \omega_{0} T
$$

$\tilde{\rho}$ is defined as in eq. (3.32) and $\gamma$ as

$$
\gamma:=-\frac{\sqrt{2 \pi}}{D S_{D}} \frac{1}{\pi^{D}} \int d \boldsymbol{c}_{1} d \boldsymbol{c}_{2} d \hat{\boldsymbol{\sigma}} \Theta\left(\boldsymbol{c}_{12} \cdot \hat{\boldsymbol{\sigma}}\right) \boldsymbol{c}_{12} \cdot \hat{\boldsymbol{\sigma}} \tilde{\rho}\left(\boldsymbol{c}_{1}\right) \tilde{\rho}\left(\boldsymbol{c}_{2}\right)(b-1) \frac{1}{2}\left(\boldsymbol{c}_{1}^{2}+\boldsymbol{c}_{2}^{2}\right)
$$

$S_{D}$ is the surface of a unit sphere in $D$ dimensions and $\omega_{0}$ the Enskog collision frequency for a classical gas of hard spheres with temperature $T=\frac{m}{2} v_{0}^{2}$, given by:

$$
\omega_{0}:=\frac{S_{D}}{\sqrt{2 \pi}} g(d) n d^{D-1} v_{0} \quad \text { and } \quad S_{D}:=\frac{2 \pi^{D / 2}}{\Gamma(D / 2)} .
$$

Taking the time derivative of eq. (3.35) we have to consider the time dependence of $\rho(\boldsymbol{v}, t)$ as well as the time dependence of $L_{l}^{\alpha}\left(\left(\frac{\boldsymbol{v}}{v_{0}(t)}\right)^{2}\right)$ via $v_{0}(t)$. The time dependence of $\rho(\boldsymbol{v}, t)$ is given by the time-evolution operator for the distribution function, and the time dependence of $v_{0}(t)$ is given by equation (3.36). After a straight forward calculation, see appendix A.4, we get

$$
\frac{d}{d t} a_{l}=\omega_{0} \gamma_{l}+l 2 \gamma \omega_{0}\left(a_{l}-a_{l-1}\right)
$$

and

$$
\begin{aligned}
\gamma_{l}=\frac{\sqrt{2 \pi}}{S_{D}} \frac{1}{\left(\begin{array}{c}
l+\alpha \\
l
\end{array}\right)} \frac{1}{\pi^{D}} \int d \boldsymbol{c}_{1} d \boldsymbol{c}_{2} d \hat{\boldsymbol{\sigma}} \Theta\left(\boldsymbol{c}_{12} \cdot \hat{\boldsymbol{\sigma}}\right) \boldsymbol{c}_{12} \cdot \hat{\boldsymbol{\sigma}} \times \\
\tilde{\rho}\left(\boldsymbol{c}_{1}\right) \tilde{\rho}\left(\boldsymbol{c}_{2}\right)(b-1) \frac{1}{2}\left(L_{l}^{\alpha}\left(\boldsymbol{c}_{1}^{2}\right)+L_{l}^{\alpha}\left(\boldsymbol{c}_{2}^{2}\right)\right) .
\end{aligned}
$$


All collision integrals $\gamma$ and $\gamma_{l}$ depend non-linearly on $a_{l}$ for all $l$ via $\tilde{\rho}\left(\boldsymbol{c}_{1}\right) \tilde{\rho}\left(\boldsymbol{c}_{2}\right)$. We mention here that our approach is equivalent to the dynamics proposed in [BrPo99], but has the advantage to give immediately the explicit time dependence of all coefficients, at least formally.

Up to now we have determined the full time dependence of the HCS in terms of the time dependence of all its moments in eqs. (3.36) and (3.39). This infinitely large system of differential equations can only be solved approximately. Only under the assumption that all neglected coefficients are small does it make sense to truncate this system of equations. It is not a priori clear which terms can be neglected. To make some progress we assume that higher order coefficients contribute less and assign to each $a_{l}$ a factor $\lambda^{l}$ and make a formal Taylor expansion around $\lambda=0$ to order $\mathcal{O}\left(\lambda^{l}\right)$. Then we set $\lambda=1$. If we now make "an approximation of $\mathcal{O}\left(\lambda^{l}\right)$ " we neglect all terms higher than $\lambda^{l}$ in eqs. (3.36) and (3.39). A posteriori the assumption has to be verified.

\section{The collision frequency}

To determine the collision frequency $\omega$ we use $\tau(t)$ which counts the number of collisions per particle, which they had to suffer until time $t . \omega$ is then given by $\frac{d}{d t} \tau(t)$ [ChCo60]. To determine $\frac{d}{d t} \tau(t)$ we use eq. (A.15) and the fact that in each collision the number of collisions that each particle has suffered increases by one and we obtain

$$
\begin{gathered}
\frac{d}{d t} \tau=\omega_{0} \gamma_{\tau}, \quad \text { and } \\
\gamma_{\tau}=\frac{\sqrt{2 \pi}}{S_{D}} \frac{1}{\pi^{D}} \int d \boldsymbol{c}_{1} d \boldsymbol{c}_{2} d \hat{\boldsymbol{\sigma}} \Theta\left(\boldsymbol{c}_{12} \cdot \hat{\boldsymbol{\sigma}}\right) \boldsymbol{c}_{12} \cdot \hat{\boldsymbol{\sigma}} \tilde{\rho}\left(\boldsymbol{c}_{1}\right) \tilde{\rho}\left(\boldsymbol{c}_{2}\right) .
\end{gathered}
$$

$\gamma_{\tau}$ depends on all $a_{l}$ and for the case that all $a_{l}=0$ we would get $\gamma_{\tau}=1$ and thus the Enskog value $\omega_{0}$. We define a time $\tilde{\tau}$ by

$$
d \tilde{\tau}=\omega_{0} d t
$$

Note that $\tilde{\tau}$ is only an approximation of $\tau$ defined in eq. (3.41), so we do not really measure time in collisions, but we will show later that the deviations of $\tilde{\tau}$ from $\tau$ remain small for not too high inelasticities. In other words we hope that the collision frequency is approximately determined by the Enskog value and corrections due to deviations from the Gaussian affecting the collision frequency are small.

\section{General considerations}

How can the dynamics be described in a state where all coefficients have already reached their asymptotic values? A simple possibility is to make the assumption that the collision frequency is given by the Enskog value $\omega_{0}$, which only depends 
on the temperature and therefore neglects deviations from the Gaussian state. We assume that $\gamma$ has reached its asymptotic value $\gamma^{*}$ for some time $t>t^{*}$ or equivalently $\tilde{\tau}>\tilde{\tau}^{*} \cdot \gamma^{*}$ is determined by the asymptotic values of $a_{l}$. Then we consider eq. (3.36) and (3.43):

$$
\begin{gathered}
\frac{d}{d t} T=-2 \gamma^{*} \omega_{0}(T(t)) T \\
\frac{d}{d t} \tilde{\tau}=\omega_{0}(T),
\end{gathered}
$$

which can we solve analytically

$$
T=\frac{T\left(\tilde{\tau}^{*}\right)}{\left(1+\omega_{0}\left(T\left(t^{*}\right)\right) \gamma^{*}\left(t-t^{*}\right)\right)^{2}}=T\left(\tilde{\tau}^{*}\right) \exp \left(-2 \gamma^{*}\left(\tilde{\tau}-\tilde{\tau}^{*}\right)\right)
$$

so that

$$
\tilde{\tau}(t)-\tilde{\tau}^{*}=\frac{1}{\gamma^{*}} \ln \left(1+\gamma^{*} \omega_{0}\left(T\left(t^{*}\right)\right)\left(t-t^{*}\right)\right) .
$$

As long as the collision frequency can be approximated by its Enskog value, eq. (3.47) provides a relation between collisional time and real time.

\section{Stationary and asymptotic values}

We are interested in the dynamics of the temperature and the coefficients as well as in the asymptotic state. The asymptotic state is characterized by the fact that the entire time dependence is given by $v_{0}(t)$ and that the coefficients $a_{l}(t)$ are stationary in time. A priori it is not clear if such a state can be found.

(i) To investigate the dynamics we integrate the full differential equation. The asymptotic values of the coefficients can then be obtained by taking the long-time limit if they become stationary in time. (ii) To discuss the stationary state we set the left hand side of eq. (3.39) equal to zero. This set of coupled and, as the case may be, non-linear equations can be solved with the numerical tool provided by the computer algebra program. Note that not all of these stationary values are necessarily dynamically stable solutions of the corresponding differential equation.

In the following we discuss (i) the dynamics and (ii) the stationary state first to order $\mathcal{O}\left(\lambda^{2}\right)$, then to order $\mathcal{O}\left(\lambda^{3}\right)$ and $\mathcal{O}\left(\lambda^{6}\right)$.

\section{Results to order 2}

In a first step we only take into account $a_{2}$ to linear order. Then the functional form of the equation for $a_{2}$ eq. (3.39) is given by

$$
\frac{d}{d t} a_{2}=\omega_{0}\left(\gamma_{2}+4 \gamma a_{2}\right) \rightarrow \frac{d}{d \tilde{\tau}} a_{2}=\gamma_{2}+4 \gamma a_{2}=: A+B a_{2}
$$


We have neglected all terms of $\mathcal{O}\left(\lambda^{3}\right)$ and $\mathrm{A}$ and $\mathrm{B}$ are constants given by the collision integrals in $\gamma$ and $\gamma_{2}$.

(i) Dynamics - By counting time in collisions per particle $a_{2}$ has, in this approximation, the time dependence

$$
a_{2}=-A / B(1-\exp (B \tilde{\tau})),
$$

so that the asymptotic value of $a_{2}$ is reached exponentially fast on a collisional time scale.

(ii) Stationary state - The stationary value $-A / B$ for $a_{2}$ coincides with the values calculated in [NoEr98b]. $A$ and $B$ are given in 2 dimensions by $A=\frac{1}{8}\left(1-3 e_{n}^{2}+2 e_{n}^{4}\right)$ and $B=\frac{1}{128}\left(30 e_{n}^{4}-5 e_{n}^{2}-32 e_{n}-57\right)$.

\section{Results to order 3}

To keep the discussion simple we take into account only $a_{2}$ and $a_{3}$ i.e. up to $\mathcal{O}\left(\lambda^{3}\right)$. We then still have to deal with equations which are linear in the coefficients $\left(a_{2}^{2}\right.$ is already of order $\mathcal{O}\left(\lambda^{4}\right)$ ). In the next section we will discuss the non-linear case up to order $\mathcal{O}\left(\lambda^{6}\right)$. We use computer algebraic programs to calculate the collisions integrals $\gamma, \gamma_{i}$ and $\gamma_{\tau}$. See appendix A.6. The results are rather lengthy and we only show here plots of the solution for a system with $e_{n}=0.9$. We consider the following equations, in which we neglect terms of higher order:

$$
\begin{gathered}
\frac{d}{d t} T=-2 \gamma \omega_{0} T \\
\frac{d}{d t} a_{2}=w_{0} \gamma_{2}+4 \gamma \omega_{0}\left(a_{2}-0\right) \\
\frac{d}{d t} a_{3}=w_{0} \gamma_{3}+6 \gamma \omega_{0}\left(a_{3}-a_{2}\right) \\
\frac{d}{d t} \tau=\omega_{0} \gamma_{\tau}, \\
\frac{d}{d t} \tilde{\tau}=\omega_{0} \quad \text { neglecting corrections of } a_{2} \text { and } a_{3} .
\end{gathered}
$$

(i) Dynamics - We have solved the simultaneous eqs. (3.50)-(3.54) numerically ${ }^{6}$ for the case $e_{n}=0.9$ and in the following we always plot time in units of $1 / \omega_{0}(T(0))$ and temperature in units of $T(0)$. We have chosen $a_{2}(0)=a_{3}(0)=0$ as initial condition. In a first step we prove that the approximation to use $\tilde{\tau}$ instead of $\tau$ can be justified (at least to this order). In fig. 3.10 a) we show the relative deviations of the true number of collisions to the approximation given by the Enskog Boltzmann value, i.e $(\tau-\tilde{\tau}) / \tau$ as function of time in a semilogarithmic plot. We see that the relative

\footnotetext{
${ }^{6}$ We have used the built-in numerical procedure dsolve of MAPLE to integrate the differential equation.
} 

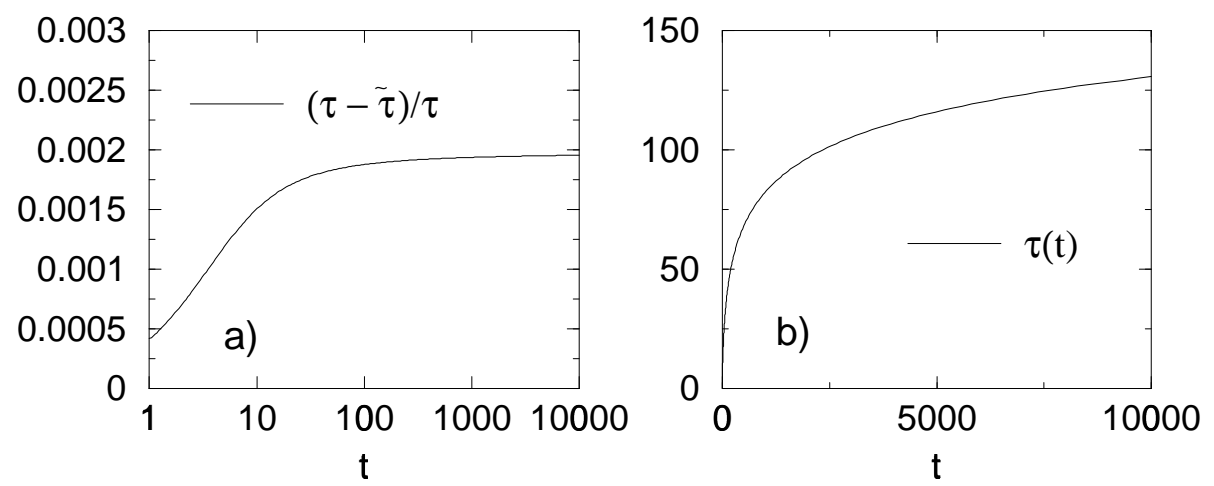

Figure 3.10.: a) Relative deviations of collisions per particle from the approximation given by the Enskog value as a function of time. b) Collisions per particle as a function of time.

deviations remain smaller than $0.2 \%$. This allows us, at least in the homogeneous cooling state, to use $\tilde{\tau}$ instead of $\tau$ in eqs. (3.46) and (3.47).

In the asymptotic state ${ }^{7}$ we get for $\gamma^{*}=0.04723$ and values from the numerical integration of eqs. (3.50)-(3.54) coincides with eq. (3.46) and (3.47) within the graphical accuracy, so we plotted here only the numerical solution. Fig. 3.10 b) shows $\tau(t)$ which has the same form as predicted in eq. (3.47).

In fig. $3.11 \mathrm{a}$ ), we show $T$ as a function of time in a double logarithmic plot. We see the well-known asymptotic time dependence $T \propto t^{-2}$. In fig. $3.11 \mathrm{~b}$ ), we show $T$ as a function of $\tau$ in a semi logarithmic plot resulting in a straight line with slope $-2 \gamma^{*}$ as predicted by eq. (3.46).

In fig. 3.12 we show the time dependence of $a_{2}$ and $a_{3}$ as a function of time a) and as a function of $\tau \mathrm{b}$ ). We see that $a_{2}$ and $a_{3}$ reach their asymptotic value on a time scale which is of the order of the time scale of the onset of the $t^{-2}$ law and that only a few collisions per particle are necessary to reach the asymptotic state for $a_{2}$ and $a_{3}$.

(ii) Stationary state- As mentioned above we calculate the stationary values by setting the l.h.s of eq. (3.50) equal to zero. In fig. 3.13 we show the results for the stationary values of $a_{2}$ and $a_{3}$ to $\mathcal{O}\left(\lambda^{3}\right)$ as well as $a_{2}$ to $\mathcal{O}\left(\lambda^{2}\right)$. As long as $e_{n}>0.6, a_{2}$ to $\mathcal{O}\left(\lambda^{2}\right)$ does not differ significantly from $a_{2}$ to $\mathcal{O}\left(\lambda^{3}\right)$ and $a_{3}$ remains small. We see stronger differences for smaller $e_{n}$ and $a_{3}$ becomes as important as $a_{2}$ which indicates stronger deviations from the Gaussian state. We also cannot assume anymore that corrections of higher orders remain small since we do not have any indication that the series is converging in the sense that the $\left|a_{l}\right|$ are small and decreasing.

${ }^{7}$ How the asymptotic value of $\gamma$, which depends on the asymptotic values of $a_{i}$, is derived, is described below. 


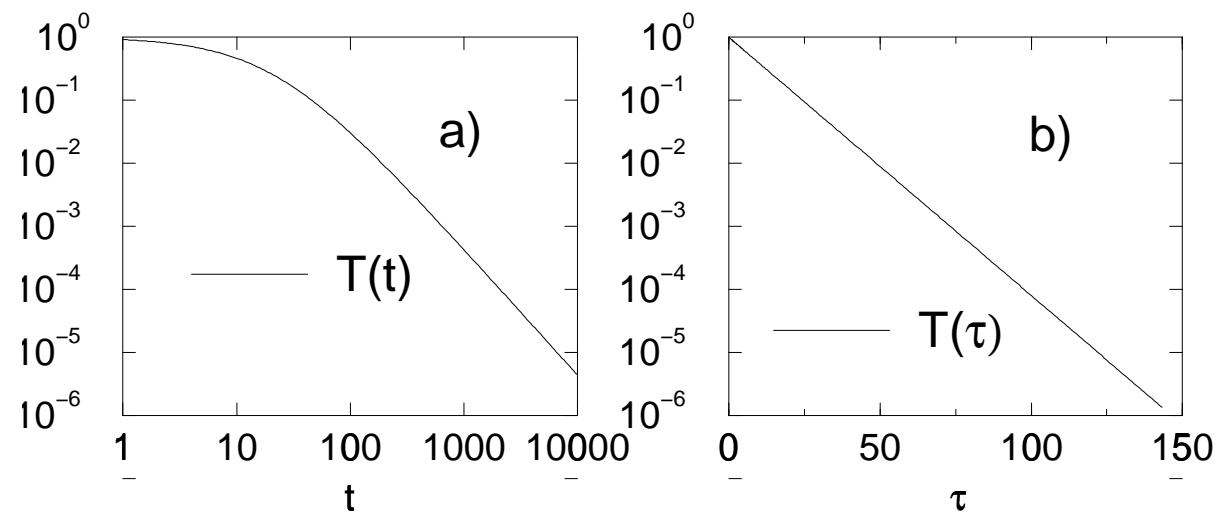

Figure 3.11.: Temperature as a function of time a) and collisions per particle b).

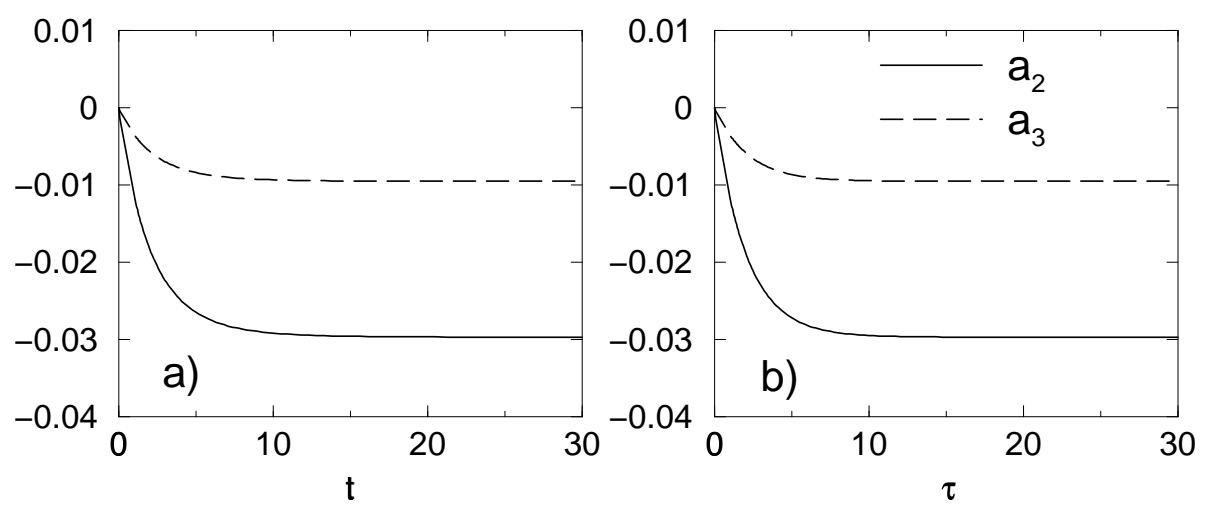

Figure 3.12.: Coefficients $a_{2}$ and $a_{3}$ as a function of time a) and collisions per particle b). 


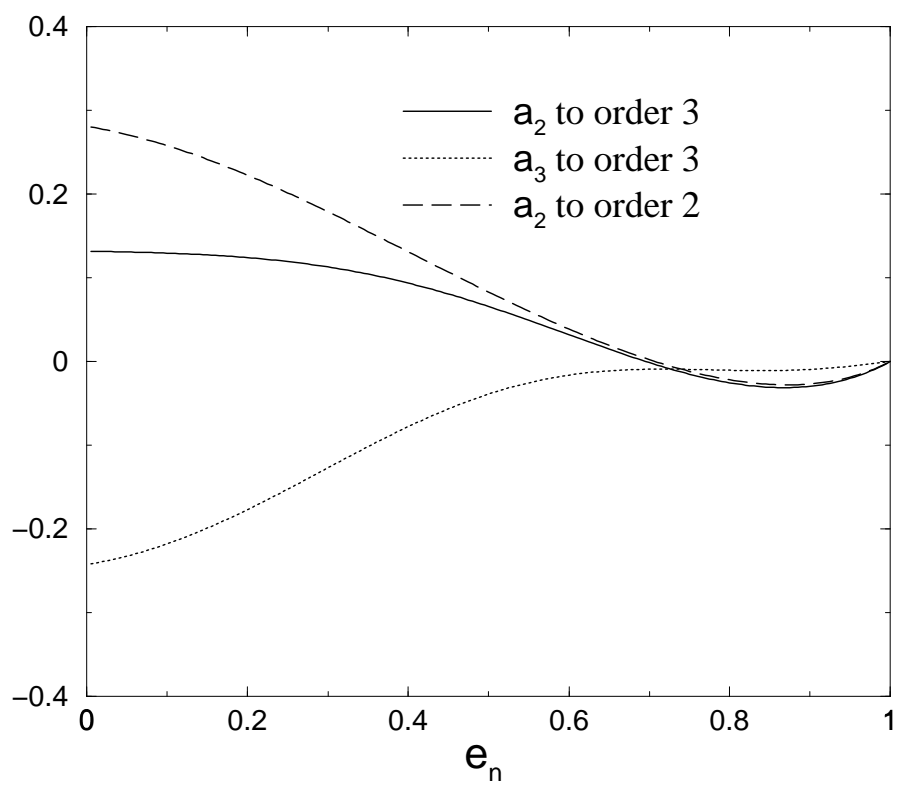

Figure 3.13.: Coefficients $a_{2}$ to $\mathcal{O}\left(\lambda^{2}\right)$, and $a_{2}$ and $a_{3}$ to $\mathcal{O}\left(\lambda^{3}\right)$ as a function of $e_{n}$

Since to $\mathcal{O}\left(\lambda^{3}\right)$ we have to deal with a set of linear equations we only find one unique solution. Considering higher orders one will find many solutions whose validity must be investigated. We will discuss this problem in the next section.

\section{Results to order 6}

In this section we go to $\mathcal{O}\left(\lambda^{6}\right)$, which is the highest order we were able to calculate with the computer algebra program.

(i) Dynamics - In fig. 3.14 we show for $e_{n}=0.8$ the dynamics for the 5 non-vanishing coefficients $a_{2}, \ldots, a_{6}$ as a function of time. We have chosen the initial condition $a_{2}(0)=\ldots=a_{6}(0)=0$. We see again a very fast decay to their asymptotic values and we can verify the assumption that $\left|a_{l}\right|>\left|a_{l+1}\right|$.

(ii) Stationary state- Again we calculate the stationary values by setting the l.h.s of eq. (3.39) equal to zero. In fig. 3.15 we show the results of the stationary values as a function of $e_{n}>0.3$. For $e_{n}>0.7$ the coefficients remain small and the expansion seems to converge in the sense that $\left|a_{l}\right|>\left|a_{l+1}\right|$ for all $l$. For $e_{n}<0.7$ the absolute values of the coefficients start to grow and seem to diverge with $e_{n}$ approaching 0.3. We will discuss the case $e_{n} \lesssim 0.3$ below in more detail. In fig. 3.16 a) and b) we compare results to order $\mathcal{O}\left(\lambda^{3}\right)$ and $\mathcal{O}\left(\lambda^{6}\right)$. As long as $e_{n}>0.6$, we do not find significant differences between the two orders, a further hint that for these values of $e_{n}$ the perturbation method works.

Since we have to deal with non-linear equations, the solution is not unique and 


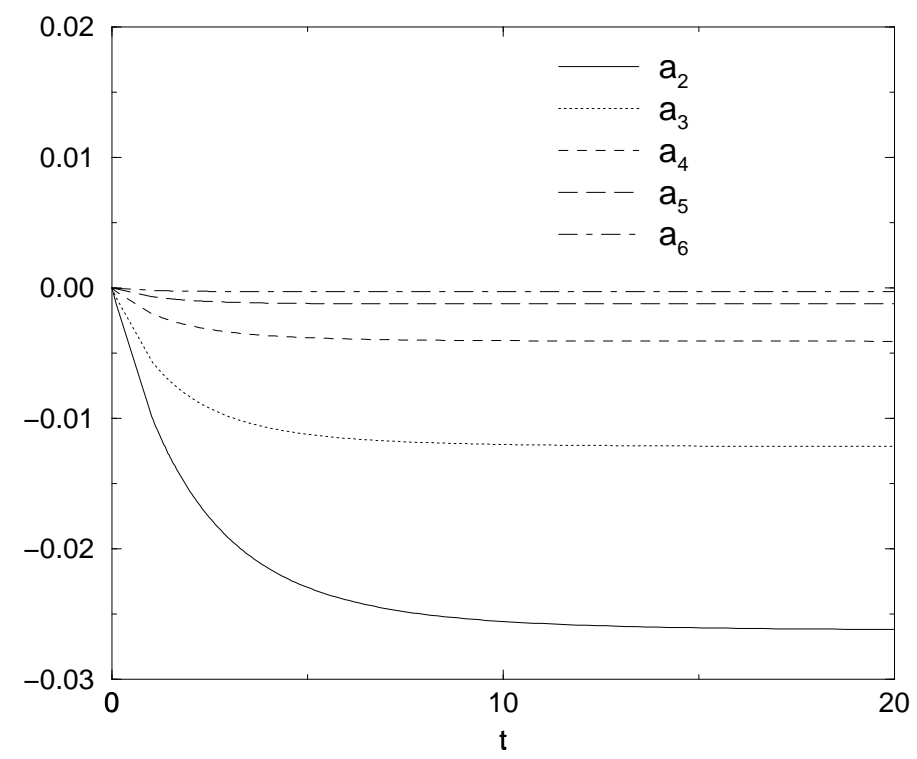

Figure 3.14.: $a_{2}, \ldots, a_{6}$ as a function of time for $e_{n}=0.8$

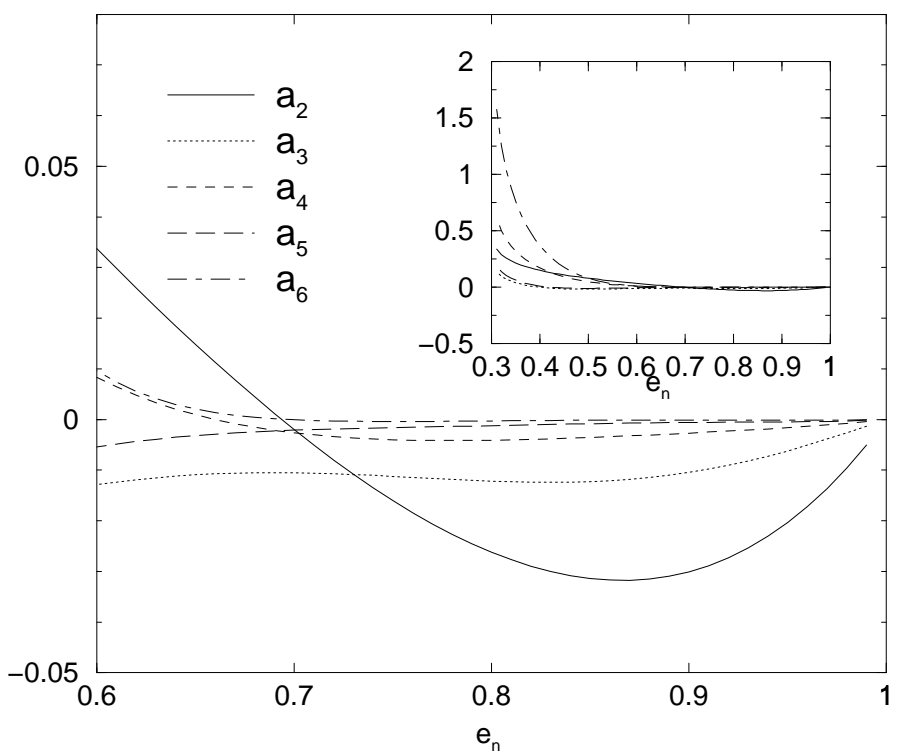

Figure 3.15.: Stationary values $a_{2}, \ldots, a_{6}$ as a function of $e_{n}$ 
e.g. for $e_{n}=0.8$ two further stationary solutions can be found, similar as in [BrPo99]. We list the values of the other coefficients for these stationary solutions:

\begin{tabular}{l|l|l} 
& Solution 1 & Solution 2 \\
\hline$a_{2}$ & 8.95 & -24.62 \\
\hline$a_{3}$ & -14.39 & -4.50 \\
\hline$a_{4}$ & 59.11 & 39.53 \\
\hline$a_{5}$ & -109.17 & 178.6 \\
\hline$a_{6}$ & 127.8 & -197.7 \\
\hline
\end{tabular}

Both solutions are dynamically unstable which we have shown by numerical integration of the corresponding differential equations (3.36) and (3.39). In addition we observe that the higher coefficients are not at all negligible so that our assumptions, which should allow us to truncate the system of differential equations, are severely violated.

The case $e_{n} \lesssim 0.3-$ We now can discuss the case $e_{n} \lesssim 0.3$. Interestingly we find for $e_{n} \lesssim 0.3$ that the coefficients do not go to an asymptotic value but diverge with time even if one starts with the initial condition $a_{2}=\ldots=a_{6}=0$, as shown in fig. $3.16 \mathrm{c}$ ). In fig. $3.16 \mathrm{~d}$ ) we show two solutions of $a_{2}$ of the corresponding equations, which are obtained by assuming that the coefficients are stationary in time. The continuous line corresponds to a dynamically stable solution, the dotted line is dynamically unstable. Both solutions join near $e_{n} \approx 0.3$, indicating that the solution becomes complex. All other coefficients $a_{3}, \ldots, a_{6}$ show the same behavior. We cannot answer if there exists no asymptotic solution for $e_{n} \lesssim 0.3$, since again in this case the assumption of small and decreasing $\left|a_{i}\right|$ is violated, and higher order terms need to be considered.

\subsubsection{Rough spheres}

In this section we investigate rough spheres and keep the assumption of homogeneity and factorization of the $N$-particle distribution function, but go beyond the approximation of a purely Gaussian state. The procedure is very similar to the case of smooth spheres. We do not investigate the dynamics but are interested in the asymptotic state. As in the case of smooth spheres in the asymptotic state the coefficients $a_{l}$ are stationary in time but in addition the asymptotic state is characterized by a constant ratio of translational to rotational energy. The time dependence is then entirely given by the time dependence of the whole kinetic energy.

\section{Dynamics of moments}

We make a double expansion of the one particle distribution function in generalized Laguerre polynomials [MaOb66] around the Gaussian. We define average velocities 

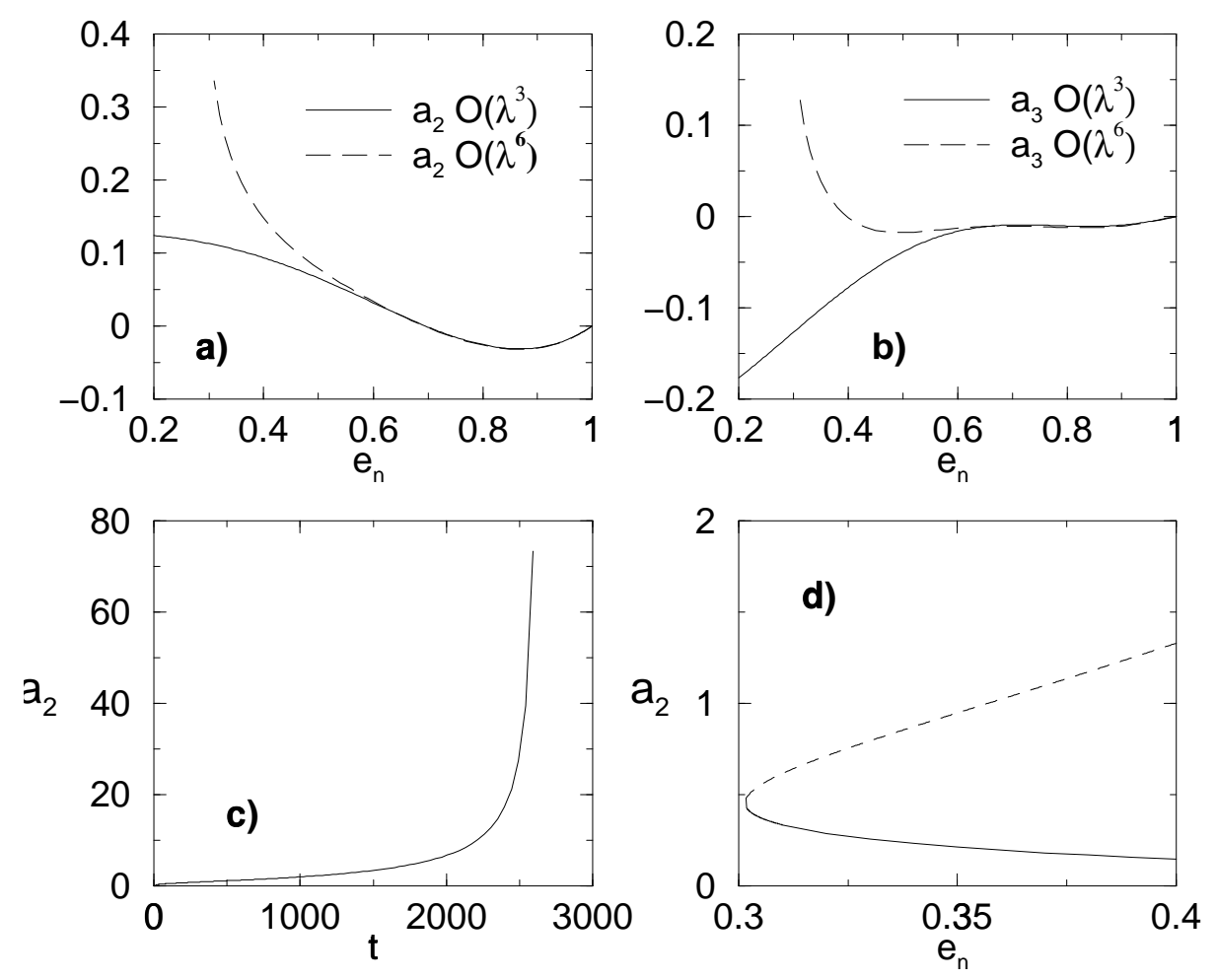

Figure 3.16.: a) Stationary value of $a_{2}$ to order $\mathcal{O}\left(\lambda^{3}\right)$ and $\mathcal{O}\left(\lambda^{6}\right)$ as function of $e_{n}$. b) As a) for $a_{3}$. c) $a_{2}(t)$ obtained by numerically solving the full differential equation to $\mathcal{O}\left(\lambda^{6}\right)$ for $e_{n}=0.2$ and initial condition $a_{2}(0)=$ $\left.\ldots=a_{6}(0)=0 . \mathrm{d}\right)$ Stable (continuous line) and unstable (dotted line) stationary values of $a_{2}$ as a function of $e_{n}$. 
$v_{0}$ and $\omega_{0}$ via the second moments of the distribution function

$$
\begin{aligned}
\int d \Gamma \rho \boldsymbol{v}_{1}^{2}=: \frac{D_{\mathrm{tr}}}{2} v_{0}^{2}(t):=\frac{D_{\mathrm{tr}}}{m} T_{\mathrm{tr}}(t) & \text { and } \\
& \int d \Gamma \rho \boldsymbol{\omega}_{1}^{2}=: \frac{D_{\mathrm{rot}}}{2} \omega_{0}^{2}(t):=\frac{D_{\mathrm{rot}}}{m} T_{\mathrm{rot}}(t),
\end{aligned}
$$

and scale linear velocities by $v_{0}(t)$ and angular velocities by $\omega_{0}(t)$. The general ansatz for the $N$-particle distribution function of the homogeneous cooling state then reads

$$
\begin{aligned}
\rho(\Gamma, t) \sim W\left(\boldsymbol{r}_{1}, \ldots \boldsymbol{r}_{N}\right) & \prod_{i=1}^{N} \rho_{i}\left(\boldsymbol{v}_{i}, \boldsymbol{\omega}_{i}, t\right), \quad \text { and } \\
\rho_{i}\left(\boldsymbol{v}_{i}, \boldsymbol{\omega}_{i}, t\right)= & \frac{1}{Z(t)} \exp \left(-\left(\frac{\boldsymbol{v}_{i}}{v_{0}(t)}\right)^{2}-\left(\frac{\boldsymbol{\omega}_{i}}{\omega_{0}(t)}\right)^{2}\right) \times \\
& \sum_{n, m=0}^{\infty} a_{n, m}(t) L_{n}^{\alpha}\left(\left(\frac{\boldsymbol{v}_{i}}{v_{0}(t)}\right)^{2}\right) L_{m}^{\beta}\left(\left(\frac{\boldsymbol{\omega}_{i}}{\omega_{0}(t)}\right)^{2}\right) .
\end{aligned}
$$

We have introduced the abbreviations $\alpha=D_{\operatorname{tr}} / 2-1$ and $\beta=D_{\text {rot }} / 2-1$. The average linear and angular velocities, $v_{0}(t)$ and $\omega_{0}(t)$, are time dependent and so are the coefficients $a_{n, m}(t)$ of the double expansion. At time $t=0$ the system is equilibrated with temperature $T$ so that $\frac{m}{2} v_{0}^{2}=T=\frac{I}{2} \omega_{0}^{2}$ and $a_{n, m}=0$.

The factor $Z(t)$ follows from the proper normalization, $\int d \boldsymbol{v}_{i} d \boldsymbol{\omega}_{i} \rho_{i}=1$,

$$
Z(t)=v_{0}^{D_{\text {tr }}} \omega_{0}^{D_{\text {rot }}} \sqrt{\pi}^{D_{\text {tr }}} \sqrt{\pi}^{D_{\text {rot }}} a_{0,0},
$$

and similar to the case of smooth spheres the orthogonality relations eq. (A.37) of the Laguerre polynomials imply $a_{1,0}=a_{0,1}=0$. It holds that

$$
a_{n, m}=\frac{1}{\left(\begin{array}{c}
n+\alpha \\
n
\end{array}\right)} \frac{1}{\left(\begin{array}{c}
m+\beta \\
m
\end{array}\right)} \int d \Gamma \rho L_{n}^{\alpha}\left(\left(\frac{\boldsymbol{v}_{1}}{v_{0}}\right)^{2}\right) L_{m}^{\beta}\left(\left(\frac{\boldsymbol{\omega}_{1}}{\omega_{0}}\right)^{2}\right) .
$$

The binomial coefficients are denoted by $\left(\begin{array}{l}a \\ b\end{array}\right)$ and we choose $a_{0,0}=1$.

Taking the time derivative of eqs. $(3.55,3.58)$ one gets the full time dependence of the homogeneous cooling state for rough spheres given by the time dependence of all its moments. The calculations are similar to the case of smooth spheres: Taking time derivatives of the right hand side of eq. (3.58) one has to take into account the time dependence of $\rho$, which is determined by $\overline{\mathcal{L}}$ as well as the time dependence of $L_{n}^{\alpha}\left(\left(\frac{\boldsymbol{v}_{1}}{v_{0}}\right)^{2}\right) L_{m}^{\beta}\left(\left(\frac{\boldsymbol{\omega}_{1}}{\omega_{0}}\right)^{2}\right)$ via $v_{0}(t)$ and $\omega_{0}(t)$, which follows from eq. (3.55). We do not investigate the dynamics here, but we are interested in the asymptotic values of the coefficients to check the validity of the Gaussian approximation. The dynamical equations look very similar to the case of smooth spheres and are found in appendix A.5. 


\section{The asymptotic values}

To get the asymptotic values we assume that all $a_{n, m}$ are stationary in time and that $v_{0} / \omega_{0}=\mu$ is constant. We obtain an infinitely large, non-linear system of equations, which reads

$$
\frac{d}{d t} a_{n, m}=0, \quad \text { and } \quad \frac{d}{d t} v_{0}=\mu \frac{d}{d t} \omega_{0}
$$

Details of the derivation and results can again be found in appendix A.5 eqs. (A.63,A.64). In principle one has to show that a constant ratio $v_{0} / \omega_{0}$ is indeed a stable solution of the corresponding dynamics. This is in general not possible, but under the assumption that all coefficients are stationary in time the full time dependence is given by the dynamics of the two temperatures. These dynamics can be determined by a similar procedure as in section 3.1. Therefore one can scale out $v_{0}$ and $\omega_{0}$ in $\left\langle i \mathcal{L} T_{\text {tr }}\right\rangle$ and $\left\langle i \mathcal{L} T_{\text {rot }}\right\rangle$. The obtained equations are of the same functional form as eqs. (3.7) with coefficients $A, B, C$ depending on the actual values of all $a_{m, n}$. These two equations are indeed solved for a constant ratio of $T_{\text {tr }} / T_{\text {rot }}$ but we can not make a prediction if it is a stable solution.

Goldshtein and Shapiro [GoSh95] propose a similar set of moment equations but they solve it only to lowest order, therefore resulting in the same asymptotic ratio $\mu$ as given in eq. (3.17).

To make any progress we truncate eq. (3.59) and take into account only $a_{n, m}$ for $n+m \leq 2$. We also neglect in eq. (3.59) products of different $a_{n, m}$, which we assume to be of higher order similar as in the case of smooth spheres. We show results for $a_{0,2}$ in fig. 3.17 for fixed $e_{n}=0.9$ as a function of $e_{t}$. Deviations from the Gaussian vanish for perfectly smooth spheres and are found to increase dramatically for $e_{t} \rightarrow-0.9$. Deviations from the Gaussian distribution are also small for perfectly rough spheres which is unexpected, because rotational degrees of freedom are coupled to translational ones and $e_{n}=0.9$. In fact deviations stay small for a broad range of values of $e_{t} \gtrsim-0.75$. We do not consider it meaningful to plot the theoretical result, once a divergence of $a_{0,2}$ has occurred. We measured $a_{0,2}$ in simulations of small systems ${ }^{8}$. Thereby we avoid clustering but have to bear with poor statistics. The simulation confirms the increase of $a_{0,2}$ around $e_{t}=-0.7$ in agreement with the perturbation expansion.

The dynamics of the system can be discussed similarly as for eq. (3.48). We consider the dynamics to linear order in $a_{0,2}, a_{2,0}$, and $a_{1,1}$ [see eq. (A.61)] and introduce the time $\tilde{\tau}$ which counts the number of collisions as in eq. (3.12) or in eq. (3.48). We obtain a system of ordinary linear differential equations, whose solution

\footnotetext{
${ }^{8}$ To estimate $a_{n, m}$ in simulations, we use eq. (3.58), but substitute for the distribution function the measured one, which is for the momenta given by $\frac{1}{N} \sum_{i=1}^{N} \delta\left(\boldsymbol{v}-\boldsymbol{v}_{i}\right) \delta\left(\boldsymbol{\omega}-\boldsymbol{\omega}_{i}\right)$. Now the integration can be performed yielding in an estimator for $a_{n, m}$. The values plotted in fig. 3.17 are averages over 10 times of a single run.
} 


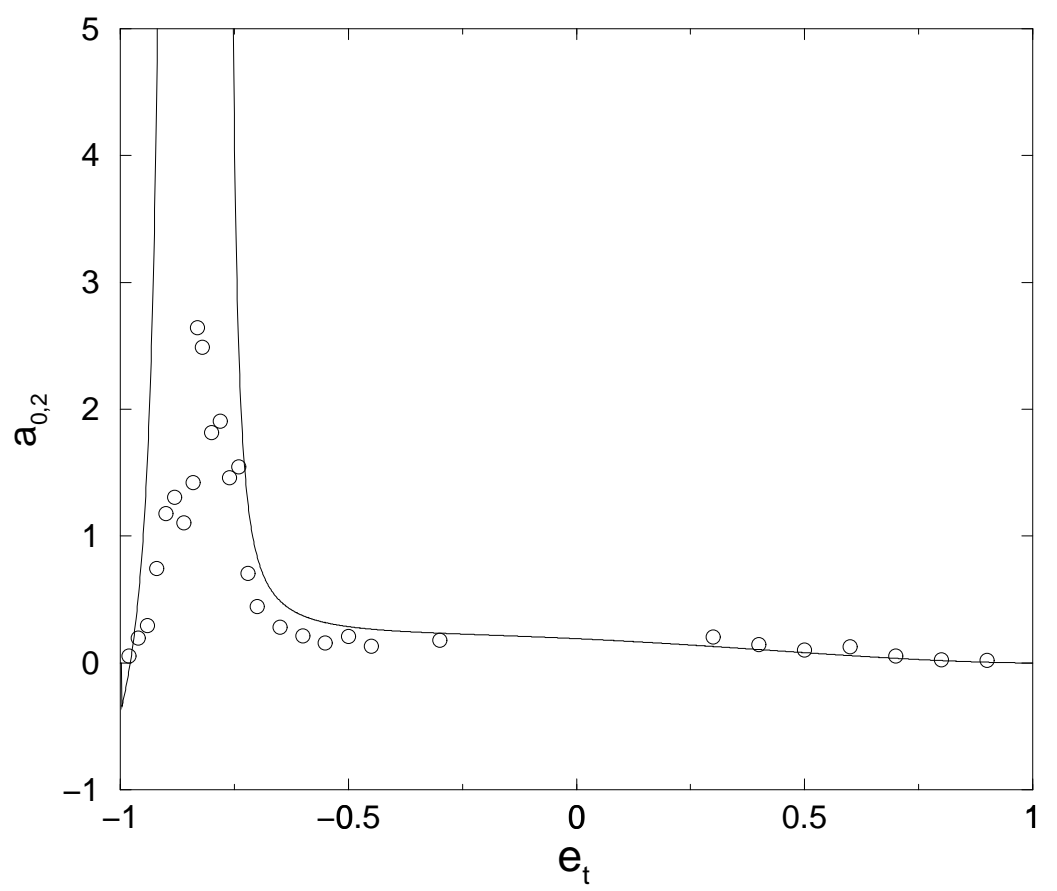

Figure 3.17.: Coefficient $a_{0,2}$ for $e_{n}=0.9$ as a function of $e_{t}$. Theory (straight line) and simulations (circles) are compared.

is exponential, indicating that the asymptotic state of the distributions function is reached exponentially fast on the time scale of collisions.

\subsection{Summary}

In chapter 3 the homogeneous cooling state for systems of smooth or rough spheres and needles has been investigated.

Gaussian approximation- In a first step we investigate rough spheres and needles and assume that the distribution function remains a Gaussian with time-dependent translational and rotational temperatures. We are able to derive two coupled differential equations for the two temperatures, whose solutions show for both systems a similar behavior: (i) A state with constant ratio of translational to rotational energy is reached exponentially fast. (ii) Once this state has been reached the whole energy decays algebraically like $t^{-2}$, i.e. according to Haff's law of homogeneous cooling. Exceptions are almost smooth spheres i.e. if the coefficients of normal and tangential restitution are such that the energy is lost mainly due to normal restitution: Then we observe an intermediate time regime where rotational energy decays with an exponent different from -2 , which depends continuously on $e_{n}$ and $e_{t}$. These results are confirmed by computer simulations of systems of small or moderate densities where 
no shearing or cluster instability is observed.

Dynamics of deviations from the Gaussian- To investigate deviations from the Gaussian state for smooth spheres we expand the distribution function in generalized Laguerre polynomials around the Gaussian state. The full dynamics of the homogeneous cooling state is expressed by the dynamics of the kinetic energy or temperature, i.e. the second moment of the distribution function, and the time dependence of the coefficients $a_{l}$ of the expansion, which corresponds to higher cumulants of the distribution function. Under the assumption that we only have to consider coefficients up to a certain order we are able to determine the dynamics approximately. We find that only a few collisions per particle are necessary to reach a state where the coefficients $a_{l}$ remain constant in time. Then the dynamics can be described by the total kinetic energy $T$ only, which follows Haff's law $\frac{d}{d t} T=-A T^{-3 / 2}$ with time-independent coefficient $A$, determined by the asymptotic values of $a_{l}$.

Validity of the Gaussian approximation- For smooth spheres we find that for $e_{n}>0.6$ deviations from the Gaussian state remain small and that the perturbative expansion seems to converge in the sense that the absolute values of the coefficients decrease with the order of the coefficients. In addition we find for this range of values of $e_{n}$ that the results for the coefficients do not depend significantly on the order of approximation. For $e_{n}<0.6$ we find stronger deviations from the Gaussian state, the absolute values of the coefficients rise and the results differ drastically from order to order of approximation. To study deviations from the Gaussian state for rough spheres, we use a similar approach. We determine the equations for the full dynamics in terms of the translational and rotational temperature and the coefficients of a double expansion in generalized Laguerre polynomials. We perturbatively calculate the asymptotic values of the first three non-vanishing coefficients by neglecting all coefficients of higher order. This perturbative approach is shown to break down for certain values of $e_{t}$ and $e_{n}$, where deviations from the Gaussian diverge. These results are supported by simulations. Analogously to the case of smooth spheres we see that at least on that level of description the asymptotic shape of the distribution function is also reached exponentially fast in the time scale of collisions.

Common features- To summarize results we find that all ${ }^{9}$ systems have in common that only a few collisions per particle are necessary to reach a state where the dynamics can be described by a single decreasing energy only, which follows Haff's law of homogeneous cooling.

\footnotetext{
${ }^{9}$ Exceptions are almost smooth spheres.
} 


\section{Inhomogeneities}

In the introduction chapter 1 we describe the mechanism that explains the shear and cluster instability in a system of smooth inelastic spheres: The collision rules of smooth spheres are such that after a collision the absolute value of the component of the relative velocity of the two colliding spheres in direction of the line connecting the center of masses is smaller than before the collision. This leads to the effect that the particles move more and more parallel and correlations in the velocities can develop. For needles collisions rules are much more complicated and the component of the relative velocity which is decreased depends strongly on the actual orientation of the needles. If the density is dilute so that needles can rotate freely between collisions, one may not expect any ordering in the velocity field. We have checked this for various coefficients of restitution and system sizes but never observed a cluster instability as long as the dimensionless density $\frac{N}{V} L^{3}$ remains below 1 . Even at higher densities it is not obvious that this happens. We will answer this question by means of simulations in the first part of this chapter.

In the second part of this chapter we investigate rough spheres in the inhomogeneous regime. We focus here only on one aspect, namely how the decay of rotational energy can be described. Also for rough spheres it holds that on average after a collision two spheres move more parallel than before. Hence correlations may build up for the translational velocity first. This might have a similar effect as for smooth spheres, namely that deviations from Haff's law occur for the translational temperature. But we have shown for the homogeneous case that the time development of the rotational temperature depends strongly on the translational temperature. Hence deviations from Haff's law of the rotational energy may be triggered by the deviations of the translational energy. Since in the HCS the constant ratio of translational to rotational energy is reached fast compared to the decay of the total energy, we hope that the rotational energy follows the translational energy by the same dependency as described in the previous chapter. We will see how these effects look like and show some encouraging results towards a hydrodynamic theory. 


\subsection{Needles}

Results of simulations of systems of needles are presented. We discuss in particular deviations from the homogeneous cooling state, which are investigated in terms if hydrodynamic quantities.

\section{Simulations}

Simulations have been performed using the event-driven algorithm described in chapter 2. The algorithm is reasonably fast as long as there are only few needles in each cell of the linked-cell structure, so that the time-consuming search for collision partners is restricted to the needles in its own and the neighboring cells. In this section we investigate dense systems, which is time consuming, because we have to choose the linear dimension of these cells to be larger than the length of a needle, so that for high densities there are many needles in each cell and the algorithm becomes slow. Hence for high densities $\left(\frac{N}{V} L^{3} \gtrsim 10\right)$ only a few simulations could be done. We show here a simulation of $N=20000$ needles in a box of length $12 L$ with $e_{n}=0.9$. To avoid inelastic collapse we use the $t_{c}$-model [LuNa98] which is also described in chapter 2.

\section{Breakdown of homogeneity; hydrodynamic quantities}

For sufficiently dense and large systems the assumption of homogeneity breaks down and deviations from Haff's law of homogeneous cooling are observed [HuAs99]. To investigate these inhomogeneities in more detail we measure hydrodynamic quantities, i.e. we define local variables like the density field, the translational and rotational flow field, and the local rotational and translational kinetic energy. In order to take local averages over small volumes, we divide the simulation box into cells whose sizes are small compared to the box size but large enough to give reasonable statistics. We choose the cell size such that on average about 25 needles are in each cell. For each cell indexed by $\alpha$ we compute the number density $\rho_{\alpha}:=\frac{1}{V_{\text {cell }}} \sum_{i \in \text { cell }_{\alpha}} 1=\langle 1\rangle_{\alpha}$, the translational energy per particle $\rho_{\alpha} E_{\alpha}^{\operatorname{tr}}=\left\langle\frac{m}{2} \boldsymbol{v}_{i}^{2}\right\rangle_{\alpha}$, and the hydrodynamic temperature $T_{\alpha}^{\mathrm{tr}}=E_{\alpha}^{\mathrm{tr}}-m \boldsymbol{U}_{\alpha}^{2} / 2$ defined by fluctuations around the flow field $\rho_{\alpha} \boldsymbol{U}_{\alpha}=\left\langle\boldsymbol{v}_{i}\right\rangle_{\alpha}$. The corresponding observables of the rotational degrees of freedom are the rotational energy per particle $E_{\alpha}^{\text {rot }}$, the hydrodynamic rotational temperature $T_{\alpha}^{\text {rot }}$ and the rotational flow field $\boldsymbol{\Omega}_{\alpha}$.

To check for spatial clustering, we compare the statistics of fluctuations of the local density, velocity, and translational energy for elastic and inelastic systems. In fig. 4.1 a) we show the histogram of the deviation of the local density $\delta \rho_{\alpha}=\rho_{\alpha} / n-1$. The initial distribution is uniform, corresponding to the equilibrium state of an elastic system. As the system develops in time with particles colliding inelastically, we observe that the distribution broadens, a clear indication that regions of large density 
a)

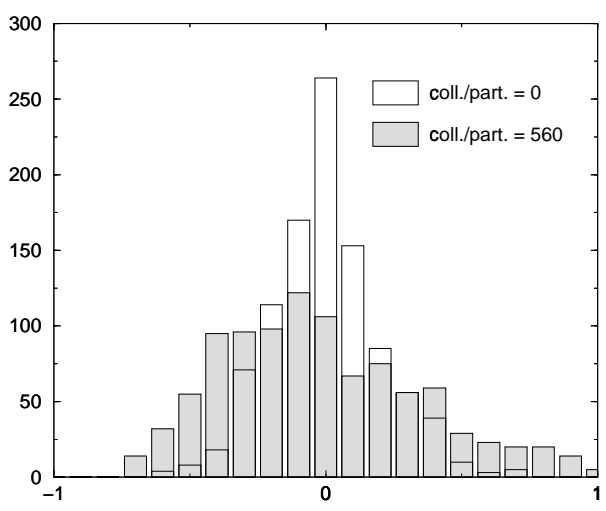

c)

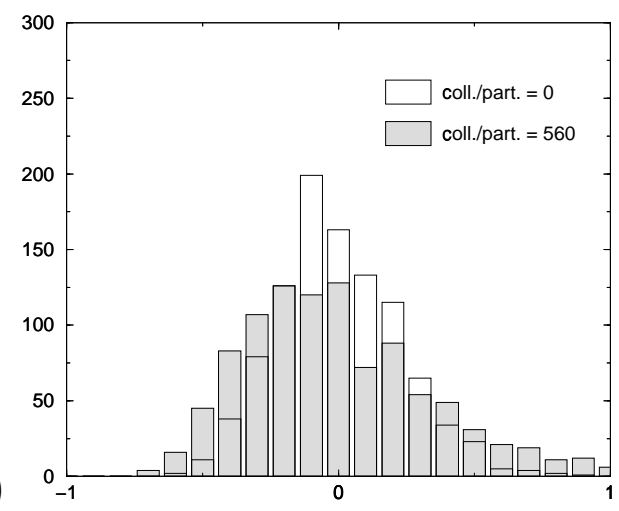

b)

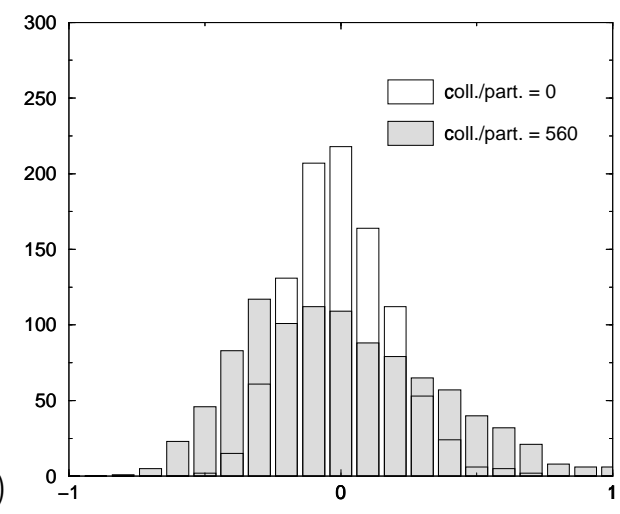

d)

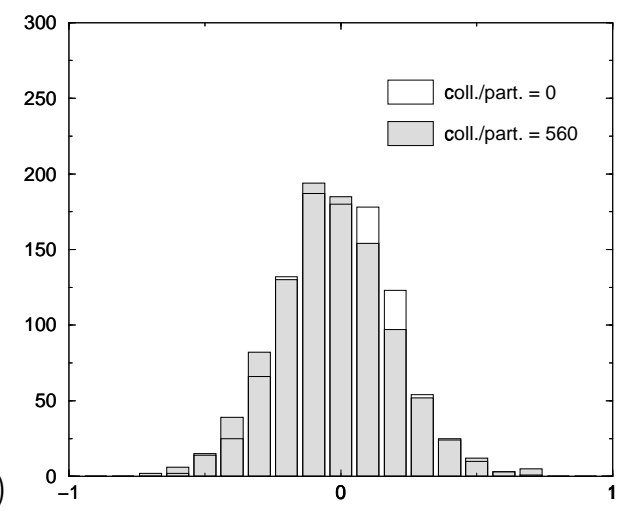

Figure 4.1.:

a) Histogram of density fluctuations in the initial state and after 560 Collisions per particle. It is obvious that regions with high density have developed.

b) Histogram of translational energy fluctuations in the initial state and after 560 Collisions per particle. Again we find that the distribution function has broadened.

c) Same as b) for the rotational energy fluctuations.

d) No significant change between the initial distribution for $Q_{\alpha}$ (defined in the text) and the distribution after 560 collisions per particle is observed. 
have developed. We measure the total translational energy $E_{\text {all }}^{\mathrm{tr}}$ per particle and the total rotational energy $E_{\text {all }}^{\text {rot }}$ per particle and plot in fig. 4.1 b) and c) histograms of the deviation of the local translational $\left(E_{\alpha}^{\mathrm{tr}} / E_{\mathrm{all}}^{\mathrm{tr}}-1\right)$ and rotational $\left(E_{\alpha}^{\mathrm{rot}} / E_{\text {all }}^{\mathrm{rot}}-1\right)$ energies, which look very similar and indicates that homogeneity breaks down also for the distribution of the energies.

\section{Orientational ordering}

To investigate local orientational ordering we compute the local average quadrupolar moment of the needles $\rho_{\alpha} Q_{\alpha}=\left\langle 3\left(\boldsymbol{u}_{z}\right)^{2}-1\right\rangle_{\alpha}$ and show in fig. $4.1 \mathrm{~d}$ ) histograms of $Q_{\alpha}$. We do not observe a significant change in the distribution. To investigate correlations between velocity of the particle and orientation we plot in fig. 4.2 histograms of $Q_{\boldsymbol{v}_{i}}=3\left(\boldsymbol{u}_{i} \hat{\boldsymbol{v}}_{i}\right)^{2}-1$ where $\hat{\boldsymbol{v}}_{i}$ is the unit vector in direction of the velocity of particle $i$. The configuration after 600 collisions per particle is compared to the initial state which correspondents to randomized orientations. In addition we plot the theoretical prediction for the histogram (straight line) which has been calculated on the assumption that rods are oriented randomly and independent of their velocity.

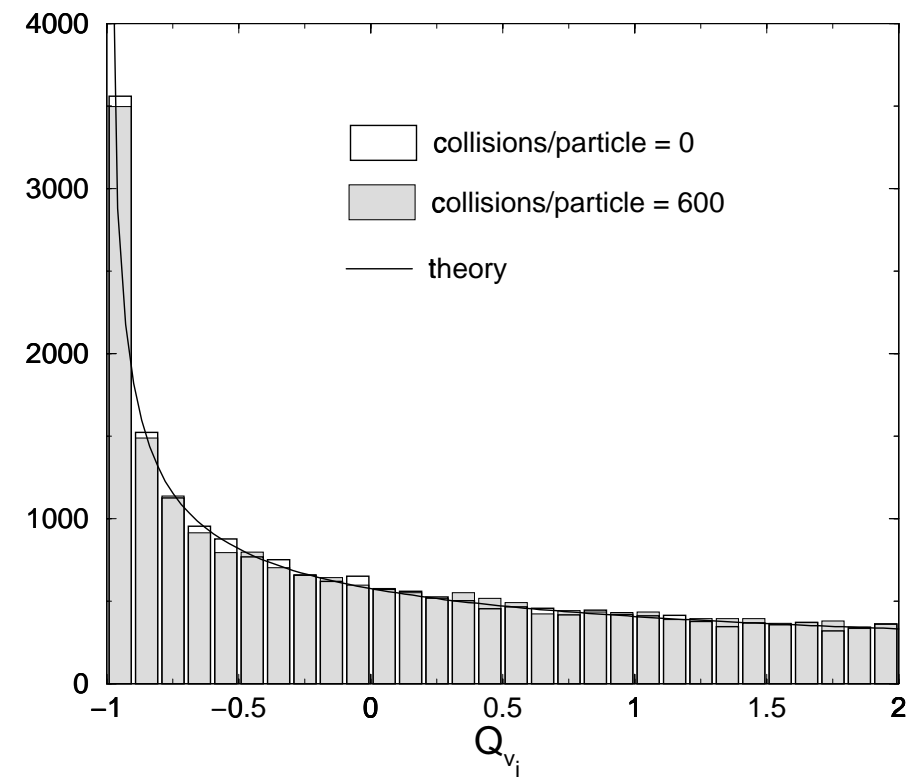

Figure 4.2.: Histogram of $Q_{\boldsymbol{v}_{i}}$. The distribution after 600 collisions per particle coincident with the initial distribution and with the prediction for randomly distributed orientations. 


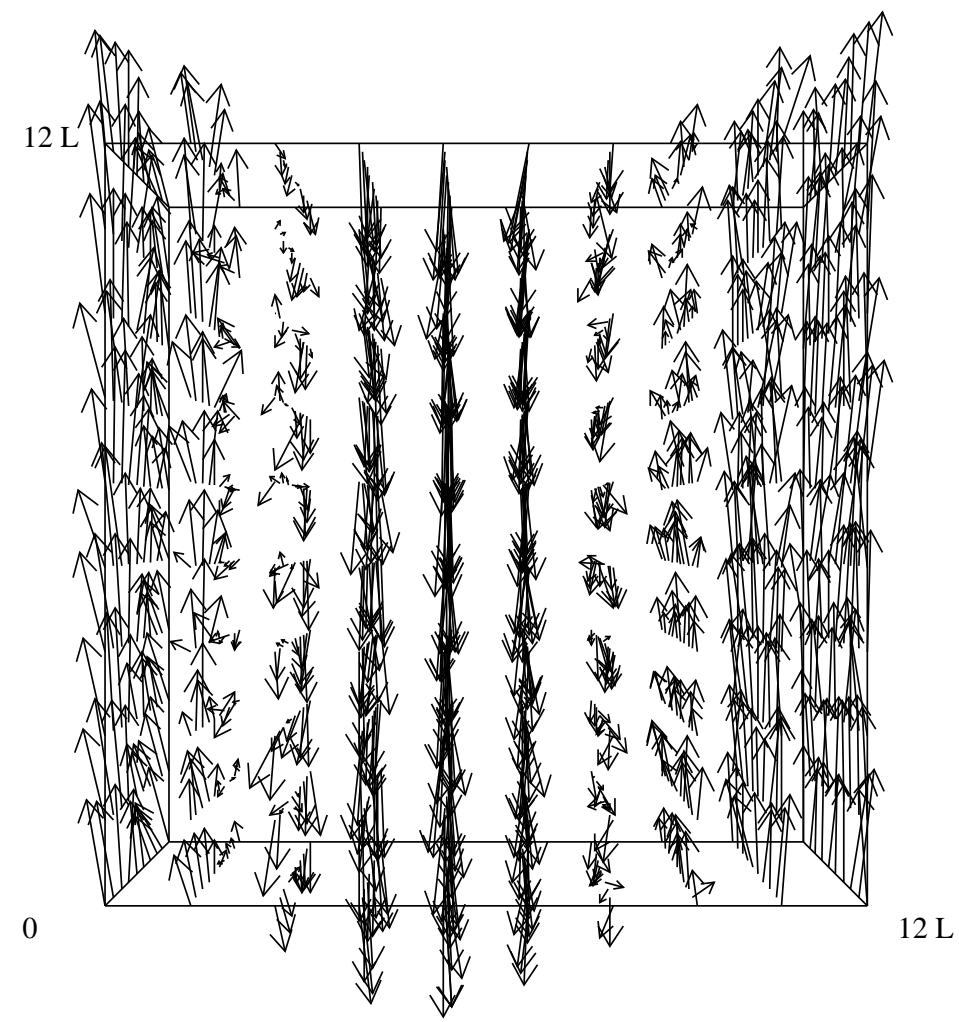

Figure 4.4.: Flow field after 600 collisions per particle 


\section{The flow field}

Inelastic hard spheres without surface roughness tend to move more and more parallel so that large scale structures in the velocity field develop. In such a state most of the kinetic energy is to be found in the energy of the flow field, whereas the energy of the fluctuations around the flow field is small. A quantitative measure for this effect [NaYo96] is the ratio of the total energy of the flow to the total internal energy of fluctuations: $S_{\mathrm{tr}}:=\left(\sum_{\alpha} \frac{m}{2} \rho_{\alpha} \boldsymbol{U}_{\alpha}^{2}\right) /\left(\sum_{\alpha} \rho_{\alpha} T_{\alpha}^{\mathrm{tr}}\right)$ and the analogous quantity $S_{\text {rot }}$ for the rotational degrees of freedom. In fig. 4.3 we show $S_{\mathrm{tr}}$ and $S_{\text {rot }}$ as a function of time, measured in collisions per particle. We observe an increase of $S_{\mathrm{tr}}$ by a factor of 50 , whereas $S_{\text {rot }}$ increases only by about $50 \%$. Hence the large scale structures in the translational flow field are much more pronounced than in the rotational flow field. In fig. 4.4 we show the flow field after 600 collisions per particle. We observe
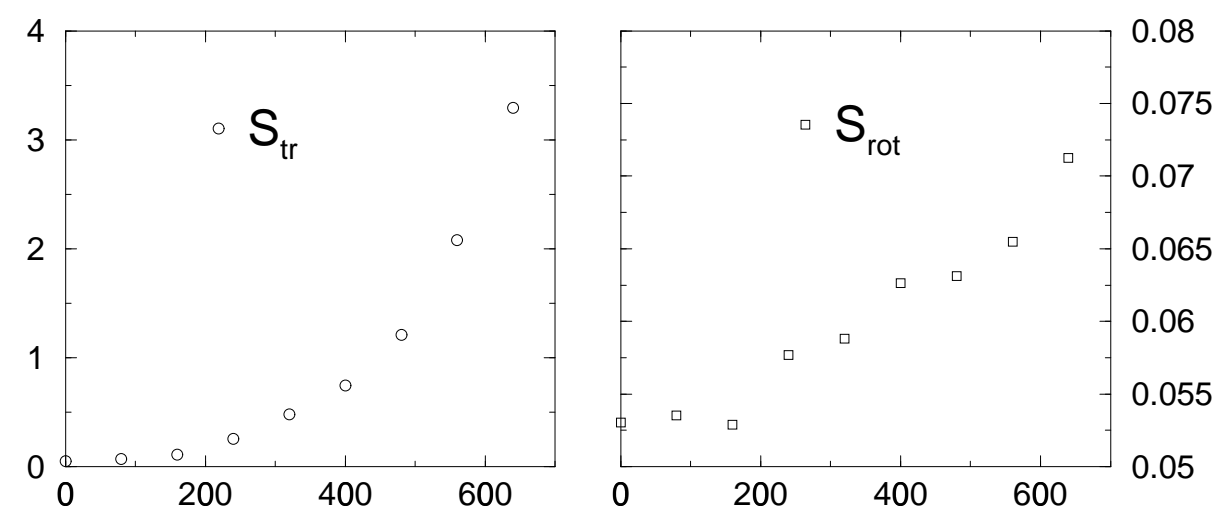

Figure 4.3.: Ratio $S_{\text {tr }}\left(S_{\text {rot }}\right)$ of the local macroscopic energy to the local temperature for the translational (rotational) degrees of freedom as a function of the number of collisions per particle.

two shear bands (note the periodic boundary conditions) in which the flow field is to a large degree aligned.

To check for correlations between the flow field and the density we plot in fig. 4.5 the components of the flow field $\boldsymbol{U}_{y}$ and $\boldsymbol{U}_{z}$ and the density fluctuations as a function of $x$ for fixed $y=6 L$ and average over 10 values of $z=1.2 L, \ldots, 12 L$. This and similar plots give no hint of strong correlation between flow field and density fluctuations.

\section{Deviations from Haff's law}

How does the organization of the flow field influence the decay of the average energy in the system? Brito and Ernst [BrEr98] have suggested a generalized Haff's law to describe the time dependence of the kinetic energy of smooth inelastically colliding 


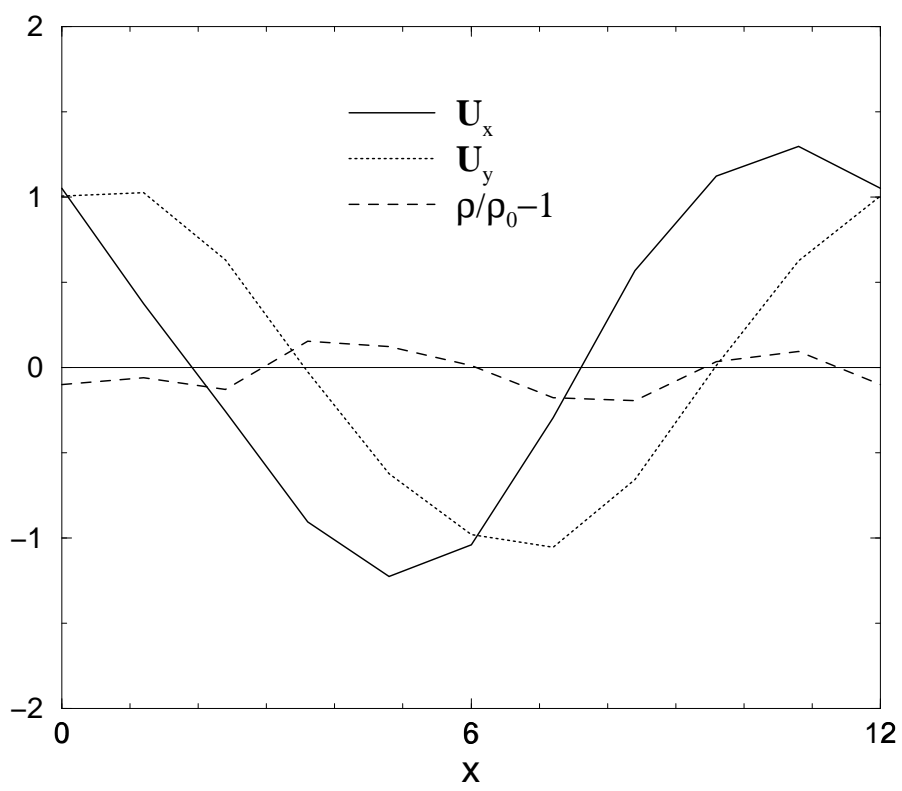

Figure 4.5.: $y$ - and $z$-component of the flow field and fluctuation of the density as a function of $x$, for fixed $y=6 L$, averaged over $10 z$-values.

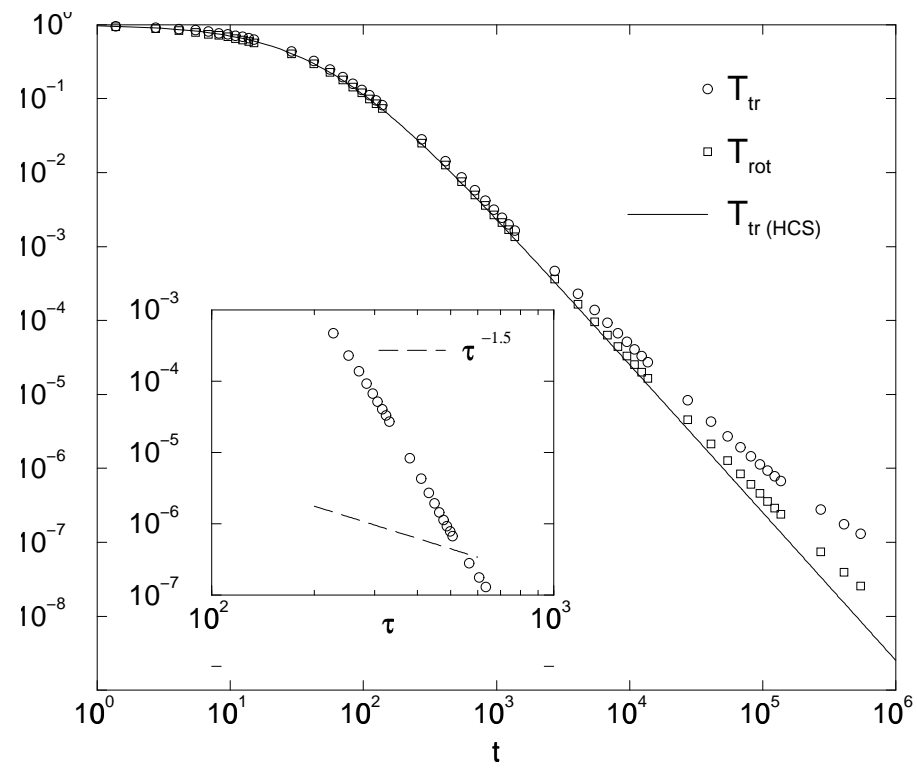

Figure 4.6.: Data of simulations of translational and rotational energy per degree of freedom as a function of time (units of $\gamma_{n} \sqrt{T_{\mathrm{tr}}(0)}$ ) compared to the numerical solution of eqs. $(3.27,3.28)$ and to $t^{-3 / 2}$. The inset shows $T_{\mathrm{tr}}$ as a function of collisions per particle $\tau$ and $\tau^{-3 / 2}$. 
spheres even in the non-homogeneous state. They found that in the late stages where one finds a well-developed flow field the energy decays like $\tau^{-D / 2}$ in $D$ dimensions. As in section 3.1.1 $\tau$ is the average number of collisions suffered by a particle within time $t$. In fig. 4.6 we compare the data of the simulation with the solution of eqs. $(3.27,3.28)$ and in the inset we plot $T_{\operatorname{tr}}$ as a function of $\tau$ and compare it to $\tau^{-3 / 2}$. We cannot confirm a $\tau^{-3 / 2}$ law, but by inspection of fig. 4.4 we see that the range of correlations are already of the order of the system size, so that finite size effects - not taken into account in the theory of Brito and Ernst - may be dominating. To simulate larger systems and longer runs has not been possible because simulations of dense systems are rather time consuming [HuAs99].

\subsection{Spheres}

The role of rotational energy in systems of inhomogeneously cooling rough spheres is investigated. We will see that deviations from the homogeneous cooling law are triggered by the translational degrees of freedom, whereas the rotational temperature follows the same law as in the homogeneous state but now has to adjust to the altered translational temperature.

\section{Simulations}

To perform simulations we use a similar method as for needles. To obtain a welldefined initial configuration we start the simulation on a regular lattice with random velocities and angular velocities chosen from a Boltzmann distribution. We equilibrate the systems by choosing $e_{n}=1$ and $e_{t}=-1$ and let the simulation run for 200 collisions per particle. Then $e_{n}$ and $e_{t}$ are switched to their desired values.

In this section we show the results of four systems with $10^{5}$ particles each. We choose $e_{n}=0.9$ and $e_{t}= \pm 0.8$ and use two different densities given in terms of the volume fraction $\Phi=\frac{4 \pi}{3} d^{3} \frac{N}{V}$. So we compare four different systems summarized in the following table:

\begin{tabular}{l|r|r} 
System & $\Phi$ & $e_{t}$ \\
\hline I & 0.1 & +0.8 \\
\hline II & 0.25 & +0.8 \\
\hline III & 0.1 & -0.8 \\
\hline IV & 0.25 & -0.8 \\
\hline
\end{tabular}

\section{Measurement of hydrodynamic fields}

In the last section we described deviations from the homogeneous cooling state in systems of smooth needles by a hydrodynamic analysis. We measure the hydrodynamic quantities: the number density $\rho_{\alpha}$, the translational energy per particle $E_{\alpha}^{\operatorname{tr}}$ and the 
hydrodynamic temperature $T_{\alpha}^{\mathrm{tr}}$ given by the fluctuations around the hydrodynamic flow field $\boldsymbol{U}_{\alpha}$ and the corresponding values for the rotational degrees of freedom.

As for needles a good indicator of long-range organization of the angular velocity field is the ratio of the total kinetic energy of the angular flow to the total rotational energy $S_{\text {rot }}:=\left(\sum_{\alpha} \frac{m}{2} \rho_{\alpha} \Omega_{\alpha}^{2}\right) /\left(\sum_{\alpha} \rho_{\alpha} E_{\alpha}^{\text {rot }}\right)$. We also measure the ratio of the total kinetic energy of the flow to the total internal energy $S_{\mathrm{tr}}$, which is known to rise strongly in systems of smooth spheres, indicating the long-range order in the translational flow field.
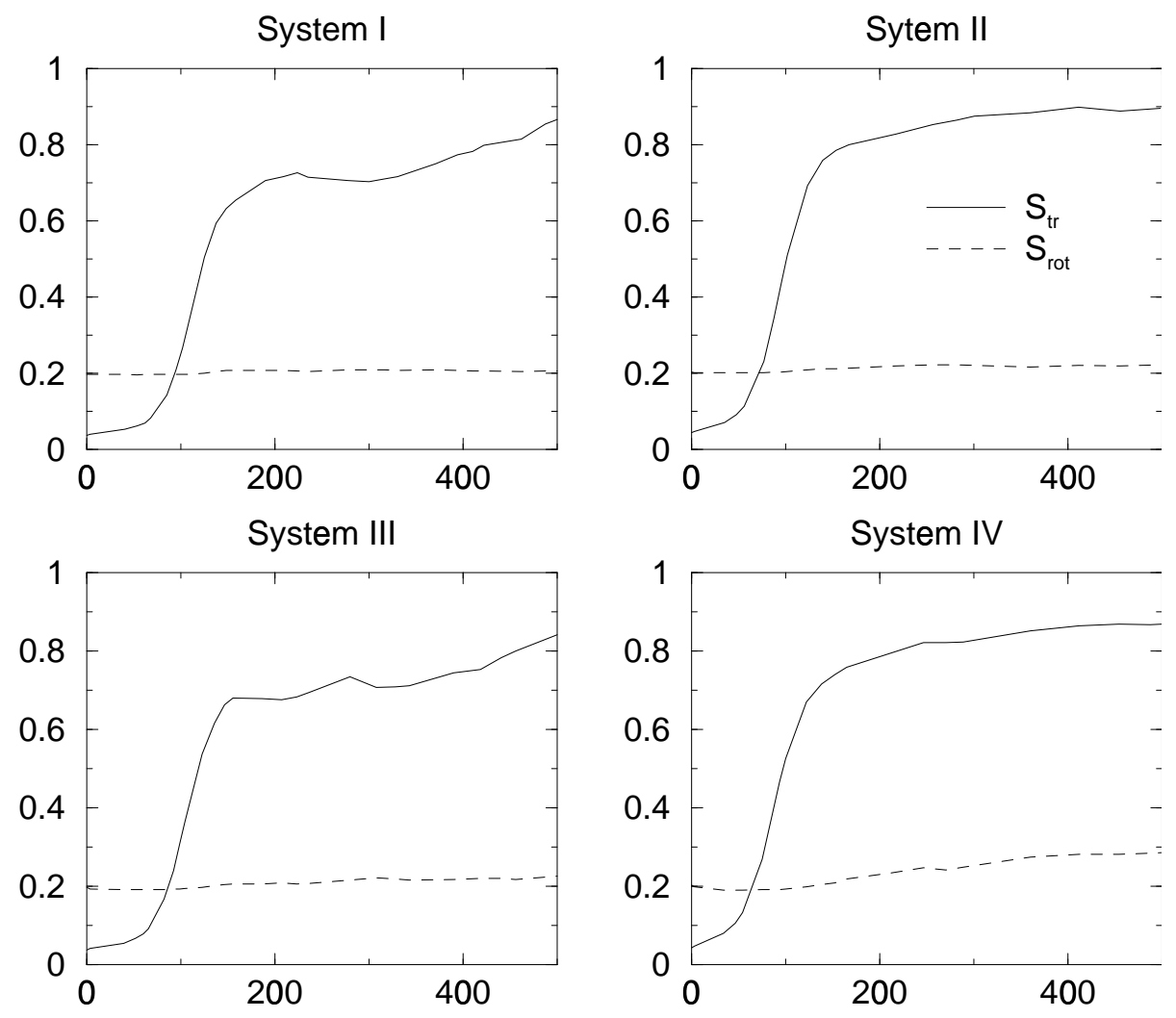

Figure 4.7.: $S_{\mathrm{tr}}$ and $S_{\text {rot }}$ as a function of collisions per particles for all four systems.

We see that $S_{\text {rot }}$ remains always of the same order of magnitude whereas $S_{\text {tr }}$ rises by a factor of about ten. While in the dilute systems we observe little change of $S_{\text {rot }}$ with time, we see larger variations for system IV. We conclude that there is no longrange ordering in the rotational degrees of freedom. Hence one may hope that the dynamics of the rotational temperature is still given by eq. (3.7) of the homogeneous cooling state. We will test this hypothesis in the next section. 


\section{The dynamics of the rotational energy}

In this section we show that for systems I-III the dynamics of the rotational temperature follows the dynamics of the translational temperature via eq. (3.7). In the following we will call this the "slaving mechanism". The flow field of particle $i$ is determined by the flow field of the corresponding box $\alpha$ and is denoted by $\boldsymbol{U}_{i}:=\boldsymbol{U}_{\alpha} \Leftrightarrow i \in \alpha$ We measure the total temperature of the system by $\frac{3}{2} T_{\mathrm{tr}}^{\exp }(t)=\frac{1}{N} \sum_{i=1}^{N} \frac{m}{2}\left(\boldsymbol{v}_{i}-\boldsymbol{U}_{i}\right)^{2}$. Here temperature denotes the energy of the fluctuations around the flow field and not the total kinetic energy. In section 3.1 we assumed to have a vanishing flow field so that in the case of the homogeneous cooling state these two definitions coincide.

To test the slaving mechanism we measure $T_{\mathrm{tr}}^{\exp }(t)$ at times $\left\{t_{j}\right\}_{j=1}^{M}$ and interpolate $^{1}$ between two measured points $t_{j}$ and $t_{j+1}$ by an algebraic decay law

$$
T_{\mathrm{tr}}^{\mathrm{int}}(t)=a_{j} t^{-b_{j}}, \quad t_{j} \leq t<t_{j+1} .
$$

$a_{j}$ and $b_{j}$ are determined by the two conditions $T_{\mathrm{tr}}^{\mathrm{int}}\left(t_{j}\right)=T_{\mathrm{tr}}^{\exp }\left(t_{j}\right)$ and $T_{\mathrm{tr}}^{\mathrm{int}}\left(t_{j+1}\right)=$ $T_{\mathrm{tr}}^{\exp }\left(t_{j+1}\right)$. We insert this piecewise defined function in eq. (3.7) and solve the following equation numerically:

$$
\frac{d}{d t} T_{\mathrm{rot}}^{\mathrm{pred}}=G B\left(T_{\mathrm{tr}}^{\mathrm{int}}(t)\right)^{3 / 2}-G C\left(T_{\mathrm{tr}}^{\mathrm{int}}(t)\right)^{1 / 2} T_{\mathrm{rot}}^{\mathrm{pred}} .
$$

We choose as initial condition for $T_{\text {rot }}^{\text {pred }}$ the measured value for $T_{\text {rot }}$ at the time $t_{1}$, where we observe first a significant deviation from the solution of homogeneous cooling state.

The results for the dilute systems are shown in fig. 4.8,4.9.

We see strong deviations from the solution of the homogeneous cooling state, whereas eq. (4.2) describes quite well the time development of the rotational temperature. For system I the rotational energy follows the step of the translational energy at time $10^{6}$ and is well-predicted. In the case of $e_{t}=-0.8$, i.e. system III, we see deviations of two orders of magnitude to the HCS-solution, while eq. (4.2) follows quite well the order of magnitude of the rotational temperature.

In figs. 4.10,4.11 we plot the dense cases. For system II we also see an encouraging result, but for system IV stronger deviations of the simulational results from the results of eq. (4.2) occur. Note that the deviations are strongest at intermediate times and "improve" at later times. It is still an open question why in this system the slaving mechanism fails. This might be traced back to a change of the distribution of the impact parameter, which can be also seen in systems of smooth spheres in the highly clustered state [BrOr99].

\footnotetext{
${ }^{1}$ We interpolate between measured points, because we need a continuously defined function to solve eq. (4.2) numerically.
} 


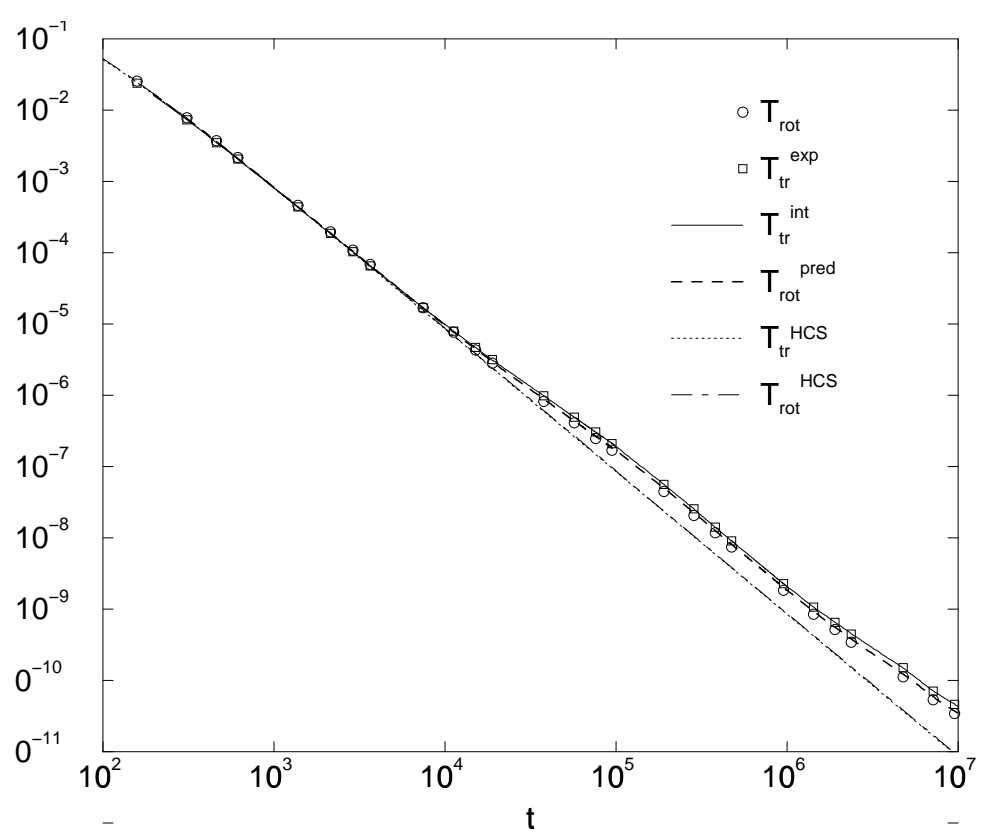

Figure 4.8.: System I: Comparison of solutions of eqs. for the HCS (3.7) and eq. (4.2) with simulations. Temperature is plotted in units of $T_{\mathrm{tr}}(0)$ and time in units of $\frac{2}{3} G T_{\mathrm{tr}}^{1 / 2}(0)$.

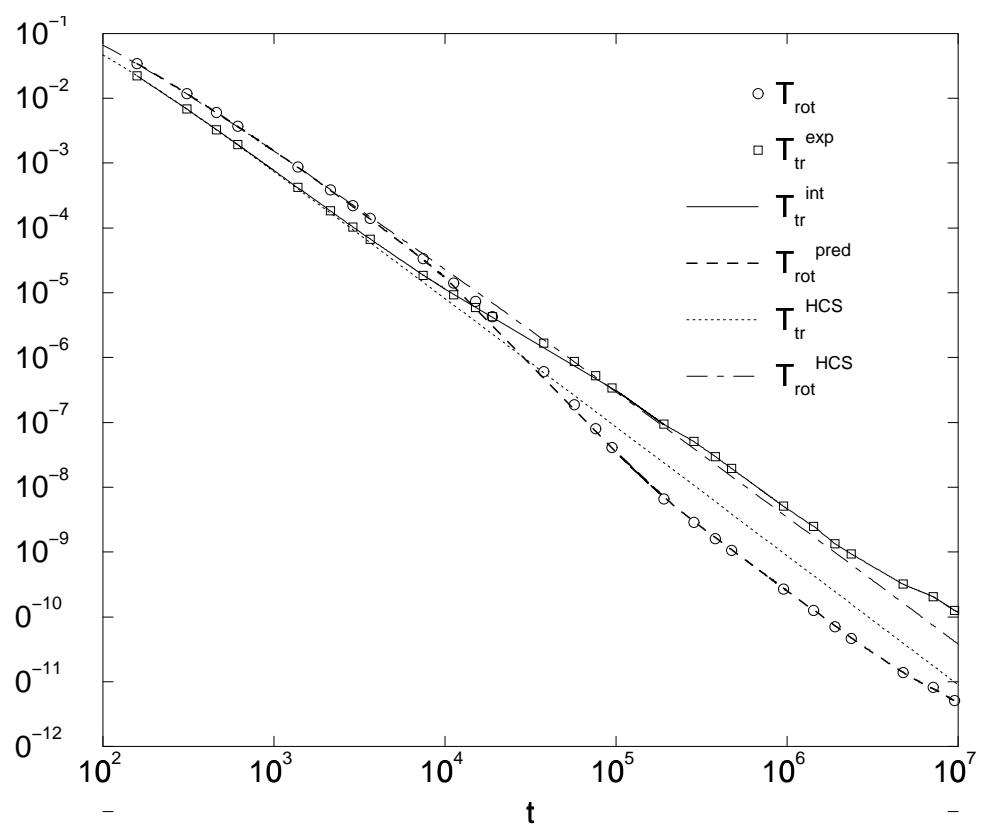

Figure 4.9.: System III: Same as fig. 4.8. 


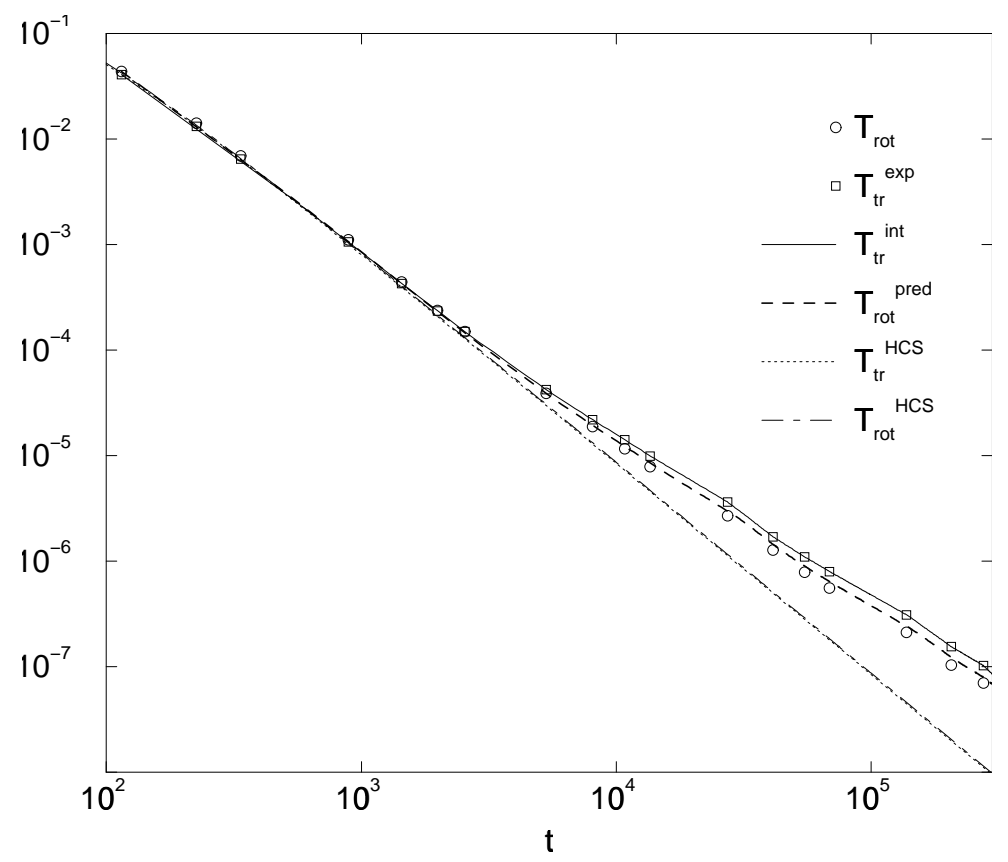

Figure 4.10.: System II: Same as fig. 4.8.

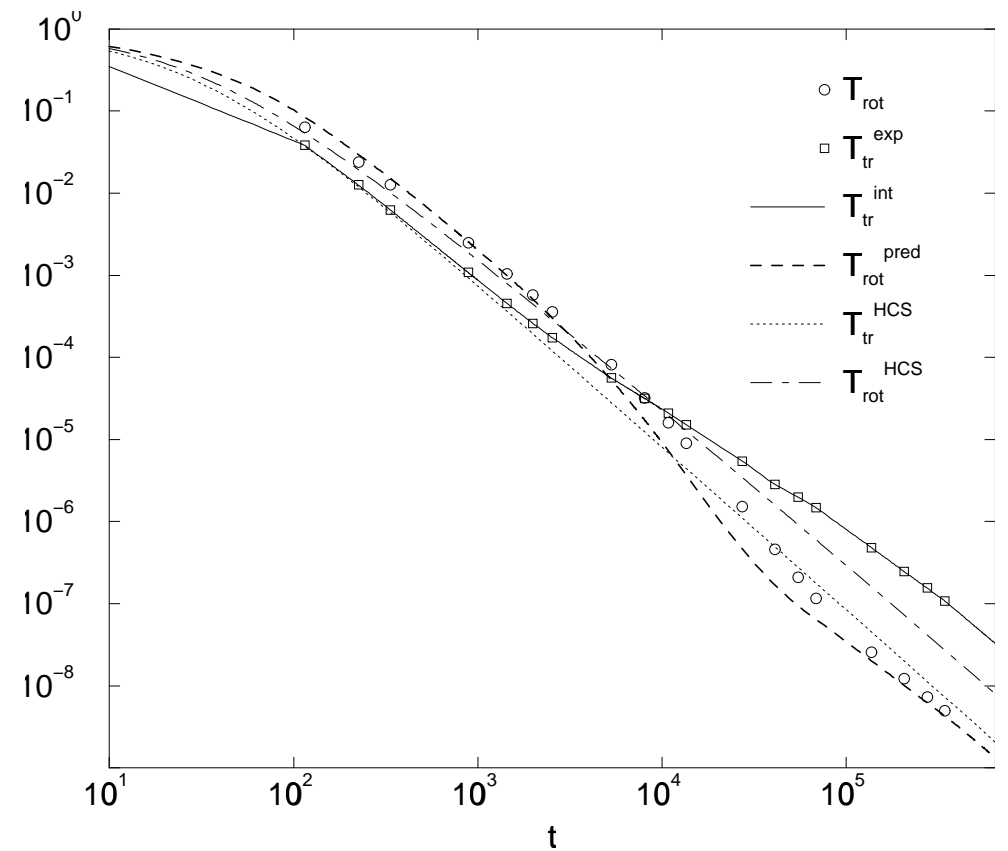

Figure 4.11.: System IV: Same as fig. 4.8. 


\subsection{Summary}

For needles we observe and investigate the breakdown of homogeneity in simulations of dense systems, where the inter-particle spacing is smaller than the length of the needles. Large-scale structures in the translational velocity field are seen to develop. Furthermore the density does not remain homogeneous but clusters form and dissolve again. These effects lead to deviations from the solution of the homogeneous cooling state on the longest times scales and a third stage of cooling is found. It is characterized by an even slower decay of the kinetic energy, most of the energy being stored in the macroscopic velocity field.

To derive generalized hydrodynamic equations for grains with rotational degrees of freedom one has to clarify the influence of angular momentum flow and rotational energy. We show by means of simulations that we do not find a significant ordering in the angular flow, neither for rough spheres nor for needles. In addition we show for rough spheres and not too high densities that the rotational temperature adjusts by the same functional dependency as in the homogeneous case - to the translational temperature, which deviates from the homogeneous cooling law.

For these reasons one may argue that in a hydrodynamic theory one could neglect angular momentum flow and restrict oneself to density, translational momentum flow and the two temperatures. Such a set of hydrodynamic equations could serve as a starting point for a stability analysis, similar to the work of Brito and Ernst [BrEr98] for smooth spheres. 


\section{The Enskog Boltzmann equation}

Starting point for many existing theories about rapid granular flow is the Enskog Boltzmann equation. The question now arises how the results obtained in this thesis are connected with the Boltzmann equation. It will become clear that on the level of approximation we made the dynamics could have been formulated equivalently by using the Enskog Boltzmann equation.

\subsection{The assumptions}

To compare our approach with the description in terms of the Enskog Boltzmann equation we recapitulate the main assumption which are made to derive the Enskog Boltzmann equation. One of many possible ways to obtain the Enskog Boltzmann equation uses the pseudo-Liouville equation eq. (2.40) derived in section 2.3. The first step is to integrate the pseudo-Liouville equation over $d \boldsymbol{r}_{3} \ldots d \boldsymbol{r}_{N} d \boldsymbol{v}_{3} \ldots d \boldsymbol{v}_{N} d \boldsymbol{\omega}_{3} \ldots d \boldsymbol{\omega}_{N}$. One obtains a equation which expresses the time evolution of the one particle distribution function in terms of the 2-particle distribution function, defined as

$$
\rho_{2}\left(\left\{\boldsymbol{v}_{i}, \boldsymbol{\omega}_{i}, \boldsymbol{r}_{i}\right\}_{i=1}^{2} t\right):=\frac{N !}{(N-2) !} \int d \boldsymbol{v}_{3} \ldots d \boldsymbol{v}_{N} d \boldsymbol{\omega}_{3} \ldots d \boldsymbol{\omega}_{N} \int d \boldsymbol{r}_{3} \ldots d \boldsymbol{r}_{N} \rho(\Gamma, t) .
$$

More general this equation is the first equation of the BBGKY-hierarchy, which expresses the time evolution of the $M$-particle distribution function in terms of the $M+1$-particle distribution function. Details can be found for example in [BBGKY, ZuMo96].

So far no approximations have been made. The Enskog Boltzmann equation is obtained by the assumption that the two particle distribution function can be expressed by the one particle distribution function and the pair correlation function defined in eq. (3.10)

$$
\rho_{2}\left(\boldsymbol{r}_{1}, \boldsymbol{v}_{1}, \boldsymbol{\omega}_{1}, \boldsymbol{r}_{2}, \boldsymbol{v}_{2}, \boldsymbol{\omega}_{2}, t\right)=g\left(\left|\boldsymbol{r}_{1}-\boldsymbol{r}_{2}\right|\right) \rho_{1}\left(\boldsymbol{r}_{1}, \boldsymbol{v}_{1}, \boldsymbol{\omega}_{1}\right) \rho_{1}\left(\boldsymbol{r}_{2}, \boldsymbol{v}_{2}, \boldsymbol{\omega}_{2}\right) .
$$

The pair correlation function describes short range correlations due to excluded volume effects. In eq. (5.2) one neglects dynamics correlations reflecting sequences of 
correlated collisions ${ }^{1}$.

We now observe that we made similar approximations. We assumed that the distribution function is of the form

$$
\rho(t)=\frac{1}{Q^{N}} W\left(\boldsymbol{r}_{1}, \ldots, \boldsymbol{r}_{N}\right) \prod_{i=1}^{N} \rho_{i}\left(\boldsymbol{v}_{i}, \boldsymbol{\omega}_{\boldsymbol{i}}, t\right) .
$$

$Q$ is chosen so that $\int \prod_{i} d \boldsymbol{r}_{i} W=Q^{N}$ and $W$ gives zero weight for overlapping configurations and $W=1$ otherwise. Again, the only correlations which are taken into account are short range excluded volume effects. We will show that both approaches yield the same result.

\subsection{Equivalence of description}

The inelastic Enskog Boltzmann equation reads [Ce95]:

$$
\begin{aligned}
& \left(\partial_{t}+\boldsymbol{v}_{1} \partial_{\boldsymbol{r}_{1}}\right) \rho_{1}(t)=n g(d) d^{D-1} \int d \boldsymbol{v}_{2} d \boldsymbol{\omega}_{2} \int d \hat{\boldsymbol{r}}\left|\boldsymbol{v}_{12} \hat{\boldsymbol{r}}\right| \Theta\left(-\boldsymbol{v}_{12} \hat{\boldsymbol{r}}\right) \times \\
& \left(\frac{\mathcal{J}}{e_{n}} \rho_{1}\left(\boldsymbol{r}_{1}, \boldsymbol{v}_{1}^{\prime \prime}, \boldsymbol{\omega}_{\mathbf{1}}^{\prime \prime}\right) \rho_{1}\left(\boldsymbol{r}_{2}, \boldsymbol{v}_{2}^{\prime \prime}, \boldsymbol{\omega}_{\mathbf{2}}^{\prime \prime}\right)-\rho_{1}\left(\boldsymbol{r}_{1}, \boldsymbol{v}_{1}, \boldsymbol{\omega}_{1}\right) \rho_{1}\left(\boldsymbol{r}_{2}, \boldsymbol{v}_{2}, \boldsymbol{\omega}_{\mathbf{2}}\right)\right) .
\end{aligned}
$$

We use the same notation as in chapter 2 section 2.3. $\mathcal{J}$ is the Jacobian of the transformation from coordinates after collision (unprimed) to coordinates before collision (double primed).

The time dependence of the average of a dynamical variable $f\left(\boldsymbol{v}_{1}, \boldsymbol{\omega}_{1}\right)$ is obtained by multiplying eq. (5.4) with $\int d \boldsymbol{v}_{1} d \boldsymbol{\omega}_{1} f\left(\boldsymbol{v}_{1}, \boldsymbol{\omega}_{1}\right)$. After a similar coordination transformation as in section 2.3 we obtain:

$$
\begin{aligned}
\partial_{t} \int d \boldsymbol{v}_{1} d \boldsymbol{\omega}_{1} f\left(\boldsymbol{v}_{1}, \boldsymbol{\omega}_{1}\right) \rho_{1}\left(\boldsymbol{r}_{1}, \boldsymbol{v}_{1}, t\right)+\partial_{\boldsymbol{r}_{1}} \int d \boldsymbol{v}_{1} d \boldsymbol{\omega}_{1} \boldsymbol{v}_{1} f\left(\boldsymbol{v}_{1}, \boldsymbol{\omega}_{1}\right) \rho_{1}\left(\boldsymbol{r}_{1}, \boldsymbol{v}_{1}, t\right) \\
=n d^{D-1} g(d) \int d \boldsymbol{v}_{1} d \boldsymbol{v}_{2} d \boldsymbol{\omega}_{1} d \boldsymbol{\omega}_{2} \int d \hat{\boldsymbol{r}}\left|\boldsymbol{v}_{12} \hat{\boldsymbol{r}}\right| \Theta\left(-\boldsymbol{v}_{12} \hat{\boldsymbol{r}}\right) \times \\
\rho_{1}\left(\boldsymbol{r}_{1}, \boldsymbol{v}_{1}, \boldsymbol{\omega}_{1}, t\right) \rho_{2}\left(\boldsymbol{r}_{2}, \boldsymbol{v}_{2}, \boldsymbol{\omega}_{\mathbf{2}}, t\right)\left(f\left(\boldsymbol{v}_{1}^{\prime}, \boldsymbol{\omega}_{1}^{\prime}\right)-f\left(\boldsymbol{v}_{1}, \boldsymbol{\omega}_{1}\right)\right) .
\end{aligned}
$$

Under the assumption of homogeneity eq. (5.5) simplifies to

$$
\begin{aligned}
& \partial_{t} \int d \boldsymbol{v}_{1} d \boldsymbol{\omega}_{1} f\left(\boldsymbol{v}_{1}, \boldsymbol{\omega}_{1}\right) \rho_{1}\left(\boldsymbol{v}_{1}, t\right) \\
&=n d^{D-1} g(d) \int d \boldsymbol{v}_{1} d \boldsymbol{v}_{2} d \boldsymbol{\omega}_{1} d \boldsymbol{\omega}_{2} \int d \hat{\boldsymbol{r}}\left|\boldsymbol{v}_{12} \hat{\boldsymbol{r}}\right| \Theta\left(-\boldsymbol{v}_{12} \hat{\boldsymbol{r}}\right) \times \\
& \rho_{1}\left(\boldsymbol{v}_{1}, \boldsymbol{\omega}_{1}, t\right) \rho_{1}\left(\boldsymbol{v}_{2}, \boldsymbol{\omega}_{\mathbf{2}}, t\right)\left(f\left(\boldsymbol{v}_{1}^{\prime}, \boldsymbol{\omega}_{1}^{\prime}\right)-f\left(\boldsymbol{v}_{1}, \boldsymbol{\omega}_{1}\right)\right) .
\end{aligned}
$$

\footnotetext{
${ }^{1} \mathrm{~A}$ more detailed discussion about this problem can be found in [NoEr97b]
} 
In appendix A.1.2 we derived in eq. (A.15) a formula for the the time dependence of the average of a dynamical variable $f\left(\boldsymbol{v}_{1}, \boldsymbol{\omega}_{1}\right)$ under the assumption of homogeneity and the assumption for the distribution function given in eq. (5.3), which reads:

$$
\begin{aligned}
\left\langle i \mathcal{L}_{+} f\right\rangle=n d^{D-1} g(d) \int d \boldsymbol{v}_{1} d \boldsymbol{v}_{2} d \boldsymbol{\omega}_{1} d \boldsymbol{\omega}_{2} \int d \hat{\boldsymbol{r}}\left|\boldsymbol{v}_{12} \hat{\boldsymbol{r}}\right| \Theta\left(-\boldsymbol{v}_{12} \hat{\boldsymbol{r}}\right) \times \\
\rho_{1}\left(\boldsymbol{v}_{1}, \boldsymbol{\omega}_{\mathbf{1}}, t\right) \rho_{1}\left(\boldsymbol{v}_{2}, \boldsymbol{\omega}_{\mathbf{2}}, t\right)\left(f\left(\boldsymbol{v}_{1}^{\prime}, \boldsymbol{\omega}_{1}^{\prime}\right)-f\left(\boldsymbol{v}_{1}, \boldsymbol{\omega}_{1}\right)\right) .
\end{aligned}
$$

We observe that the r.h.s of eq. (5.6) coincides with the r.h.s of eq. (5.7). Hence under the assumption of homogeneity the description in terms of the Enskog Boltzmann equation yields the same results as derived in this thesis. 


\section{Conclusions and outlook}

In this work we try to elucidate the role of rotational degrees of freedom in a system of freely cooling granular particles. To gather more general aspects we consider rough spherical particles and, as an example for non-spherical particles, needles. We choose the simplest collision rules which allow for exchange of translational and rotational energy. The dynamics is formulated in terms of a pseudo-Liouville operator and we use an ED-algorithm to perform simulations. For the considered systems two regimes are found, both of which are characterized by common features:

\section{The homogeneous cooling regime}

Initially, all systems are prepared in a way that the spatial correlations are those of an equilibrium ensemble of a classical system of non-dissipative particles. The velocities and angular velocities are distributed according to a Maxwellian determined by two temperatures $T_{\mathrm{tr}}$ and $T_{\text {rot }}$ respectively. For short times or not too high inelasticities the system remains spatially homogeneous, but changes arise in the distribution function for the momenta. The changes are twofold: (i) Due to the dissipative character of collisions the average kinetic energy decreases and (ii) the shape of the distribution function is no longer Maxwellian with a single temperature. The latter is expressed by a ratio, in general different from one, of the average translational energy to the average rotational energy and the emergence of higher cumulants, which are zero in a pure Maxwellian state. The main result is that for all systems the change of the distribution function to an asymptotic fixed shape ${ }^{1}$ is fast compared to the decay of the average energy. For example only a few collisions per particle are necessary to reach a state where the ratio of rotational and translational energy remains constant in time. Once the system is characterized by a fixed distribution function, the decay of the mean energy $E$ follows Haff's law of homogeneous cooling $E \propto t^{-2}$, a slow algebraic decay.

${ }^{1}$ Here fixed shape means that if all velocities are scaled with the actual mean velocity the distribution function is invariant in time. 


\section{The inhomogeneous regime}

For sufficiently large inelasticities, densities and system sizes the assumption of homogeneity breaks down. Large scale structures in the translational velocity field arise, whereas long-range organization in the rotational velocity field seems not to occur, or is at least much less pronounced. Clusters form and dissolve again, so also the density does not remain homogeneous. In this state most of the translational energy is stored in the translational velocity field and deviations from Haff's law are found. Also the rotational energy does no longer obey Haff's. For spheres we could show that it adjusts to the now different translational temperature according to the law found for the homogeneous cooling state.

\section{Outlook}

Analytical extensions as well as further investigations using simulations are desirable.

\section{- Analytical analysis}

Since a hydrodynamic analysis has successfully explained the shear and cluster instabilities of smooth spheres, it is promising to apply hydrodynamic methods to the systems considered here. A first step would be to assume that the velocity distribution function in the HCS remains Gaussian. For spheres this can be justified at least for coefficients of restitution, for which the system remains almost Gaussian as described in section 3.2.2. Furthermore motivated by the results of simulations in chapter 4 the angular momentum field could be neglected. The remaining hydrodynamic quantities would be density, translational flow field and the two temperatures. A gradient or Chapman Enskog expansion [ChCo60, BrDu98] around the HCS (the HCS is described in detail in section 3.1) would give transport coefficients and constitutive relations for the hydrodynamic equations. A linear stability analysis would show for which inelasticities, densities, and system sizes one could expect the onset of clustering. Correlation functions could be calculated by fluctuational hydrodynamics as was done for smooth spheres in [NoEr97].

A further possible extension would be to explain why the organization of angular momentum remains negligible by additionally taking into account the equations for the angular momentum field. A second possible way to elucidate the role of the angular momentum field would be a calculation of the angular velocity-angular velocity correlation function on a more microscopic level using a ring collision expansion as suggested in [NoEr98] for the velocity velocity correlation function.

\section{- Simulations}

By means of simulations the conditions under which clustering occurs could be tested more systematically. Measurement of correlations functions would give further insight into the structure of the angular momentum field .

There is still an open field concerning modeling of collisions. More realistic colli- 
sion rules include Coulomb friction. Therefore the formalism presented in this thesis can be applied and work along these lines is in progress [He99]. Rods with finite diameter may show different density-dependent effects, even the statics is not trivial and phase transitions to ordered phases are observed. 


\section{A. Appendix}

\section{A.1. Calculations for spheres}

\section{A.1.1. An example}

As an example, we explain the main steps to calculate $\left\langle i \mathcal{L} E_{\mathrm{tr}}\right\rangle_{\mathrm{HCS}}$ of eq. (3.6) in 2D. We define the configuration integral

$$
Q^{N}:=\int \prod_{i=1}^{N} d \boldsymbol{r}_{i} W\left(\boldsymbol{r}_{1}, \ldots, \boldsymbol{r}_{N}\right) .
$$

The proper normalized $N$-particle distribution function for the HCS-state reads

$$
\begin{array}{r}
\rho_{\mathrm{HCS}}(\Gamma ; t)=\frac{1}{Q^{N}}\left(\frac{m}{2 \pi T_{\mathrm{tr}}(t)}\right)^{N}\left(\frac{I}{2 \pi T_{\mathrm{rot}}(t)}\right)^{N / 2} \times \\
\quad \exp \left[-\sum_{i=1}^{N}\left(\frac{m}{2 T_{\mathrm{tr}}(t)} \boldsymbol{v}_{i}^{2}+\frac{I}{2 T_{\mathrm{rot}}(t)} \omega_{i}^{2}\right)\right] .
\end{array}
$$

The angular velocity is a scalar in two dimensions, but a vector in more than two dimensions. Free streaming does not change the energy, so we have to take into account only the collision operator $\mathcal{L}_{+}$and compute

$$
\begin{aligned}
&\left\langle i \mathcal{L}_{+} E_{\mathrm{tr}}\right\rangle_{\mathrm{HCS}}= \frac{1}{2} \sum_{i \neq j} \int d \Gamma \rho_{\mathrm{HCS}}(\Gamma ; t) i \mathcal{T}_{+}^{(i j)} \frac{1}{N} \sum_{k=1}^{N} \frac{m}{2} \boldsymbol{v}_{k}^{2}= \\
& \frac{1}{2 N} \sum_{i \neq j} \int d \Gamma \rho_{\mathrm{HCS}}(\Gamma ; t) i \mathcal{T}_{+}^{(i j)} \frac{m}{2}\left(\boldsymbol{v}_{i}^{2}+\boldsymbol{v}_{j}^{2}\right) .
\end{aligned}
$$

The binary-collision operator $\mathcal{T}_{+}^{(i j)}$ gives a contribution only if either $k=i$ or if $k=j$. Next, we introduce two $\delta$-functions,

$$
\begin{aligned}
\left\langle i \mathcal{L}_{+} E_{\mathrm{tr}}\right\rangle_{\mathrm{HCS}}=\frac{1}{2 N} \sum_{i \neq j} \int d \Gamma \int d \boldsymbol{R}_{1} d \boldsymbol{R}_{2} \delta\left(\boldsymbol{R}_{1}-\boldsymbol{r}_{i}\right) \delta\left(\boldsymbol{R}_{2}-\boldsymbol{r}_{j}\right) \times \\
\rho_{\mathrm{HCS}}(\Gamma ; t) i \mathcal{T}_{+}^{(i j)} \frac{m}{2}\left(\boldsymbol{v}_{i}^{2}+\boldsymbol{v}_{j}^{2}\right)
\end{aligned}
$$


which allows us to replace $\boldsymbol{r}_{i}$ by $\boldsymbol{R}_{1}$ and $\boldsymbol{r}_{j}$ by $\boldsymbol{R}_{2}$ in $\mathcal{T}_{+}^{(i j)}$. We define the pair correlation function $g\left(\left|\boldsymbol{R}_{1}-\boldsymbol{R}_{2}\right|\right)$ by

$$
\frac{N}{V^{2}} g\left(\left|\boldsymbol{R}_{1}-\boldsymbol{R}_{2}\right|\right):=\frac{1}{N} \sum_{i \neq j} \frac{1}{Q^{N}} \int \prod_{k=1}^{N} d \boldsymbol{r}_{k} W\left(\boldsymbol{r}_{1}, \ldots, \boldsymbol{r}_{N}\right) \delta\left(\boldsymbol{R}_{1}-\boldsymbol{r}_{i}\right) \delta\left(\boldsymbol{R}_{2}-\boldsymbol{r}_{j}\right) .
$$

Eq. (A.5) is used to rewrite eq. (A.4) in terms of the pair correlation function. Integration over all velocities and angular velocities with index $k$ and $i \neq k \neq j$ gives 1 due to normalization. We get

$$
\begin{aligned}
\left\langle i \mathcal{L}_{+} E_{\mathrm{tr}}\right\rangle_{\mathrm{HCS}}= & \frac{N}{2 V^{2}}\left(\frac{m}{2 \pi T_{\mathrm{tr}}(t)}\right)^{2} \frac{I}{2 \pi T_{\mathrm{rot}}(t)} \int d \omega_{1} d \omega_{2} d \boldsymbol{R}_{1} d \boldsymbol{R}_{2} d \boldsymbol{v}_{1} d \boldsymbol{v}_{2} \\
& \exp \left(-\frac{m}{2 T_{\mathrm{tr}}(t)}\left(\boldsymbol{v}_{1}^{2}+\boldsymbol{v}_{2}^{2}\right)-\frac{I}{2 T_{\mathrm{rot}}(t)}\left(\omega_{1}^{2}+\omega_{2}^{2}\right)\right) \times \\
& g(\boldsymbol{r})\left|\boldsymbol{v}_{12} \cdot \hat{\boldsymbol{r}}\right| \Theta\left(-\boldsymbol{v}_{12} \cdot \hat{\boldsymbol{r}}\right) \delta(|\boldsymbol{r}|-d) \Delta E_{\mathrm{tr}} .
\end{aligned}
$$

The loss of translational energy of two colliding particles is denoted by $\Delta E_{\mathrm{tr}}$. We use the abbreviation $\boldsymbol{R}_{1}-\boldsymbol{R}_{2}=\boldsymbol{r}=r \hat{\boldsymbol{r}}$ and neglect non-contributing terms linear in $\Omega$ so that $\Delta E_{\text {tr }}$ is given by

$$
\begin{aligned}
\Delta E_{\mathrm{tr}}=\frac{m}{2}\left[2 \eta _ { t } ( \eta _ { t } - 1 ) \left(\boldsymbol{v}_{12}^{2}-\right.\right. & \left.\left(\boldsymbol{v}_{12} \cdot \hat{\boldsymbol{r}}\right)^{2}\right)- \\
& \left.(1 / 2)\left(1-e_{n}^{2}\right)\left(\boldsymbol{v}_{12} \cdot \hat{\boldsymbol{r}}\right)^{2}+(1 / 2) \eta_{t}^{2} d^{2}\left(\omega_{1}+\omega_{2}\right)^{2}\right] .
\end{aligned}
$$

To perform the remaining integrations we substitute

$$
\begin{aligned}
\Omega & =\frac{1}{\sqrt{2}}\left(\omega_{1}+\omega_{2}\right), & \omega & =\frac{1}{\sqrt{2}}\left(\omega_{1}-\omega_{2}\right), \\
\boldsymbol{V} & =\frac{1}{\sqrt{2}}\left(\boldsymbol{v}_{1}+\boldsymbol{v}_{2}\right), & \boldsymbol{v} & =\frac{1}{\sqrt{2}}\left(\boldsymbol{v}_{1}-\boldsymbol{v}_{2}\right), \\
\boldsymbol{r} & =\boldsymbol{R}_{1}-\boldsymbol{R}_{2}, & \boldsymbol{R} & =\boldsymbol{R}_{1} .
\end{aligned}
$$

The Jacobian determinant for the above transformation is 1 . Integration over $\omega, \boldsymbol{V}$ and $\boldsymbol{R}$ all give the value 1 due to normalization. We are left with

$$
\begin{aligned}
&\left\langle i \mathcal{L}_{+} E_{\mathrm{tr}}\right\rangle_{\mathrm{HCS}}=\frac{N}{V} \frac{m}{2 \pi T_{\mathrm{tr}}(t)}\left(\frac{2 I}{2 \pi T_{\mathrm{rot}}(t)}\right)^{1 / 2} \int d \Omega d \boldsymbol{r} d \boldsymbol{v} \\
& \exp \left(-\frac{m \boldsymbol{v}^{2}}{2 T_{\mathrm{tr}}(t)}-\frac{I \Omega^{2}}{2 T_{\mathrm{rot}}(t)}\right) g(\boldsymbol{r})|\boldsymbol{v} \cdot \hat{\boldsymbol{r}}| \Theta(-\boldsymbol{v} \cdot \hat{\boldsymbol{r}}) \delta(|\boldsymbol{r}|-d) \times \\
& \frac{m}{2}\left[2 \eta_{t}\left(\eta_{t}-1\right)\left(\boldsymbol{v}^{2}-(\boldsymbol{v} \cdot \hat{\boldsymbol{r}})^{2}\right)-(1 / 2)\left(1-e_{n}^{2}\right)(\boldsymbol{v} \cdot \hat{\boldsymbol{r}})^{2}+(1 / 2) \eta_{t}^{2} d^{2} \Omega^{2}\right] .
\end{aligned}
$$


The integration over $|\boldsymbol{r}|$ yields $d g(d)$. Choosing e.g. $\boldsymbol{r}$ to point along the $x$-axis, the integrals over linear and angular velocities can easily be done as moments of a Gaussian distribution. The result is independent of $\hat{\boldsymbol{r}}$, so that the integration over $\hat{\boldsymbol{r}}$ gives $2 \pi$. Finally we obtain the result of eq. (3.8).

\section{A.1.2. General collision integral}

More general we assume that the $N$-particle distribution function is of the form

$$
\rho(t)=\frac{1}{Q^{N}} W\left(\boldsymbol{r}_{1}, \ldots, \boldsymbol{r}_{N}\right) \prod_{i=1}^{N} \rho_{i}\left(\boldsymbol{v}_{i}, \boldsymbol{\omega}_{\boldsymbol{i}}, t\right) .
$$

$Q$ is chosen so that $\int \prod_{i} d \boldsymbol{r}_{i} W=Q^{N}$ and $W$ gives zero weight for overlapping configurations and $W=1$ otherwise. All $\rho_{i}$ are identical for all $i$. We consider an observable depending only on velocities $f_{i}\left(\boldsymbol{v}_{i}, \boldsymbol{\omega}_{i}\right)$ of one particle or equivalently $g=\frac{1}{N} \sum_{i} f_{i}$, since all particles are equally distributed.

We calculate the time derivative of the non-equilibrium expectation value of $g$ defined in eq. (2.33). We will use the identity eq. (2.41). Since the free streaming part of the Liouville operator does not change velocities, we have to calculate only

$$
\left\langle i \mathcal{L}_{+} g\right\rangle(t)=\frac{1}{2} \sum_{i \neq j} \int d \Gamma \rho i \mathcal{T}_{i}^{(i j)} g .
$$

Step by step we make the same calculations as above for the translational energy of all particles. First we introduce two $\delta$-functions $\int d \boldsymbol{R}_{1} d \boldsymbol{R}_{2} \delta\left(\boldsymbol{R}_{1}-\boldsymbol{r}_{i}\right) \delta\left(\boldsymbol{R}_{2}-\boldsymbol{r}_{j}\right)$ and replace $\boldsymbol{r}_{i}$ and $\boldsymbol{r}_{j}$ by $\boldsymbol{R}_{1}$ and $\boldsymbol{R}_{2}$ in $\mathcal{T}_{+}^{(i j)}$ to rewrite eq. (A.12) in terms of the pair correlation function $g(r)$ also defined above in eq. (A.5)

$$
\begin{aligned}
&\left\langle i \mathcal{L}_{+} g\right\rangle=\frac{N}{2 V^{2}} \int d \boldsymbol{\omega}_{1} d \boldsymbol{\omega}_{2} d \boldsymbol{v}_{1} d \boldsymbol{v}_{2} d \boldsymbol{R}_{1} d \boldsymbol{R}_{2} \\
& \rho_{1} \rho_{2} g(|\boldsymbol{r}|)\left|\boldsymbol{v}_{12} \hat{\boldsymbol{r}}\right| \Theta\left(-\boldsymbol{v}_{12} \hat{\boldsymbol{r}}\right) \delta(|\boldsymbol{r}|-d)(b-1)\left(f_{1}+f_{2}\right) .
\end{aligned}
$$

We introduced the notation $\boldsymbol{r}=\boldsymbol{R}_{1}-\boldsymbol{R}_{2}$. Making a coordinate transformation $\boldsymbol{R}=\boldsymbol{R}_{1}$ and $\boldsymbol{r}=\boldsymbol{R}_{1}-\boldsymbol{R}_{2}$ with Jacobian determinant 1 we get after integration over $\boldsymbol{R}$ and $|\boldsymbol{r}|$

$$
\begin{aligned}
&\left\langle i \mathcal{L}_{+} g\right\rangle=\frac{N}{V} d^{D_{\mathrm{tr}}-1} g(d) \int d \boldsymbol{\omega}_{1} d \boldsymbol{\omega}_{2} d \boldsymbol{v}_{1} d \boldsymbol{v}_{2} d \hat{\boldsymbol{r}} \\
& \rho_{1} \rho_{2}\left|\boldsymbol{v}_{12} \hat{\boldsymbol{r}}\right| \Theta\left(-\boldsymbol{v}_{12} \hat{\boldsymbol{r}}\right)(b-1) \frac{1}{2}\left(f_{1}+f_{2}\right) .
\end{aligned}
$$

All particles are interchangeable so that we can replace $\frac{1}{2}\left(f_{1}+f_{2}\right)$ by $f_{1}$

$$
\left\langle i \mathcal{L}_{+} f_{1}\right\rangle=\frac{N}{V} d^{D_{\operatorname{tr}}-1} g(d) \int d \boldsymbol{\omega}_{1} d \boldsymbol{\omega}_{2} d \boldsymbol{v}_{1} d \boldsymbol{v}_{2} d \hat{\boldsymbol{r}} \rho_{1} \rho_{2}\left|\boldsymbol{v}_{12} \hat{\boldsymbol{r}}\right| \Theta\left(-\boldsymbol{v}_{12} \hat{\boldsymbol{r}}\right)(b-1) f_{1}
$$




\section{A.2. Calculations for needles}

In this appendix we present some of the detailed calculations for needles. As a first step we express the orientation of the rods in spherical coordinates

$$
\boldsymbol{u}_{i}=\left(\sin \left(\theta_{i}\right) \cos \left(\phi_{i}\right), \sin \left(\theta_{i}\right) \sin \left(\phi_{i}\right), \cos \left(\theta_{i}\right)\right) .
$$

Thus the canonical momenta (translational and rotational) are given by

$$
\boldsymbol{p}_{i}=m \boldsymbol{v}_{i}, \quad p_{\theta_{i}}=I \dot{\theta}_{i}, \quad p_{\phi_{i}}=I \dot{\phi}_{i} \sin ^{2} \theta .
$$

In the following calculation it will be useful to express $\dot{\boldsymbol{u}}_{i}$ in terms of canonical momenta

$$
\dot{\boldsymbol{u}}_{i}=\frac{p_{\theta_{i}}}{I} \boldsymbol{e}_{\theta_{i}}+\frac{p_{\phi_{i}}}{\sin \theta_{i} I} \boldsymbol{e}_{\phi_{i}}
$$

where $\boldsymbol{e}_{\theta_{i}}$ and $\boldsymbol{e}_{\phi_{i}}$ are orthogonal unit vectors in $\theta_{i^{-}}$and $\phi_{i^{-}}$direction. The translational and rotational kinetic energies per particle are then given by

$$
E_{\mathrm{tr}}=\frac{1}{N} \sum_{i=1}^{N} \frac{1}{2 m} \boldsymbol{p}_{i}^{2}, \quad E_{\mathrm{rot}}=\frac{1}{N} \sum_{i=1}^{N}\left(\frac{1}{2 I} p_{\theta_{i}}^{2}+\frac{1}{2 I \sin ^{2} \theta_{i}} p_{\phi_{i}}^{2}\right) .
$$

We want to calculate non-equilibrium expectation values with the normalized probability distribution given in eq. (3.5). Again we consider as an example the translational energy per particle $E_{\mathrm{tr}}=\frac{1}{N} \sum_{i=1}^{N} \frac{m}{2} \boldsymbol{v}_{i}^{2}$.

$$
\begin{aligned}
&\left\langle i \mathcal{L}_{+} E_{\mathrm{tr}}\right\rangle=\frac{1}{V^{N}} \frac{1}{(4 \pi)^{N}} \frac{1}{\left(2 \pi M T_{\mathrm{trans}}\right)^{3 N / 2}} \frac{1}{\left(2 \pi I T_{\mathrm{rot}}\right)^{N}} \times \\
& \frac{1}{2} \sum_{m \neq n} \int \prod_{j=1}^{N} d \boldsymbol{r}_{j} d \phi_{j} d \theta_{j} d \boldsymbol{p}_{j} d p_{\theta_{j}} d p_{\phi_{j}} \\
& \quad \exp \left[-N E_{\mathrm{tr}} / T_{\mathrm{tr}}(t)-N E_{\mathrm{rot}} / T_{\mathrm{rot}}(t)\right] i \mathcal{T}_{+}^{(n m)} E_{\mathrm{tr}} .
\end{aligned}
$$

Similar to the calculation for the spheres we see that the binary-collision operator $\mathcal{T}_{+}^{(n m)}$ gives a contribution only if either $i=n$ or if $i=m$. We can sum over $N(N-1)$ identical integrals and get

$$
\begin{aligned}
& \frac{N-1}{2 V^{2}} \frac{1}{(4 \pi)^{2}} \frac{1}{\left(2 \pi m T_{\mathrm{tr}}\right)^{3}} \frac{1}{(2 \pi I T \operatorname{rot})^{2}} \int \prod_{j=1}^{2} d \boldsymbol{r}_{j} d \phi_{j} d \theta_{j} d \boldsymbol{p}_{j} d p_{\theta_{j}} d p_{\phi_{j}} \\
& \exp \left[-E_{\mathrm{tr}}^{12} / T_{\mathrm{tr}}(t)-E_{\mathrm{rot}}^{12} / T_{\mathrm{rot}}(t)\right]\left|\frac{d}{d t}\right| \boldsymbol{r}_{12}^{\perp}|| \Theta\left(-\frac{d}{d t}\left|\boldsymbol{r}_{12}^{\perp}\right|\right) \times \\
& \Theta\left(L / 2-\left|s_{12}\right|\right) \Theta\left(L / 2-\left|s_{21}\right|\right) \delta\left(\left|\boldsymbol{r}_{12}^{\perp}\right|-0^{+}\right) \Delta E_{\mathrm{tr}}^{12} .
\end{aligned}
$$


$E_{\mathrm{tr}}^{12}\left(E_{\mathrm{rot}}^{12}\right)$ is the sum of the translational (rotational) kinetic energy of particle 1 and 2 and with $\Delta E_{\mathrm{tr}}^{12}$ we denote the change of the translational kinetic energy of particle 1 and 2 in a collision:

$$
\begin{aligned}
\Delta E_{\mathrm{tr}}^{12} & =\frac{\left(\boldsymbol{p}_{1}-\boldsymbol{p}_{2}\right) \cdot \Delta \boldsymbol{p}}{m}+\frac{\Delta \boldsymbol{p}^{2}}{m}, \\
\Delta \boldsymbol{p} & =-\frac{1+e_{n}}{2} \frac{1}{\frac{1}{m}+\frac{s_{12}^{2}}{2 I}+\frac{s_{21}^{2}}{2 I}}\left(\boldsymbol{V} \cdot \boldsymbol{u}_{\perp}\right) \boldsymbol{u}_{\perp} .
\end{aligned}
$$

$\boldsymbol{V}$ is the relative velocity of the contact points defined in eq. (2.16).

We introduce relative coordinates $\boldsymbol{r}_{12}=\boldsymbol{r}_{1}-\boldsymbol{r}_{2}$ and $\boldsymbol{r}=\boldsymbol{r}_{1}$ and the variables

$$
\begin{aligned}
z & :=\boldsymbol{r}_{12} \cdot \boldsymbol{u}_{\perp}, \\
a & :=\boldsymbol{r}_{12} \cdot \boldsymbol{u}_{1}-\frac{\boldsymbol{u}_{1} \cdot \boldsymbol{u}_{2}}{\sqrt{1-\left(\boldsymbol{u}_{1} \cdot \boldsymbol{u}_{2}\right)^{2}}} \boldsymbol{r}_{12} \cdot \boldsymbol{u}_{1}^{\perp}=-s_{12}, \\
b & :=\frac{1}{\sqrt{1-\left(\boldsymbol{u}_{1} \cdot \boldsymbol{u}_{2}\right)^{2}}} \boldsymbol{r}_{12} \cdot \boldsymbol{u}_{1}^{\perp}=s_{21} .
\end{aligned}
$$

The Jacobian of the transformation is given by $\sqrt{1-\left(\boldsymbol{u}_{1} \cdot \boldsymbol{u}_{2}\right)^{2}}$. We remark that $\frac{d}{d t}\left|\boldsymbol{r}_{12}^{\perp}\right|=\boldsymbol{V} \cdot \boldsymbol{u}_{\perp} \operatorname{sign}\left(\boldsymbol{r}_{12} \cdot \boldsymbol{u}^{\perp}\right)$ and we find again the relative velocity of the contact points $\boldsymbol{V}=\frac{\boldsymbol{p}_{12}}{m}-a \dot{\boldsymbol{u}}_{1}-b \dot{\boldsymbol{u}}_{2}$ given in the new coordinates. Integration over $\boldsymbol{r}$ gives $V$ and integration over $z$ gives the sum of two $\Theta$-functions $\Theta\left( \pm \boldsymbol{V} \cdot \boldsymbol{u}_{\perp}\right)^{1}$. Next one introduces relative and center of mass momenta, again as dimensionless variables:

$$
\begin{array}{rlrl}
\chi & :=\frac{1}{\sqrt{2 m T_{\text {trans }}}}\left(\boldsymbol{p}_{1}-\boldsymbol{p}_{2}\right), & \boldsymbol{\gamma}:=\frac{1}{\sqrt{2 m T_{\text {trans }}}}\left(\boldsymbol{p}_{1}+\boldsymbol{p}_{2}\right), \\
\tilde{p}_{\theta_{i}}:=\frac{p_{\theta_{i}}}{\sqrt{I T_{\text {rot }}}}, & \tilde{p}_{\phi_{i}}:=\frac{p_{\phi_{i}}}{\sqrt{I T_{\text {rot }} \sin \theta_{i}} .}
\end{array}
$$

The integration over $\gamma$ can be done and the result is proportional to

$$
\begin{gathered}
\sum_{p= \pm 1} \int d a d b d \phi_{1} \sin \theta_{1} d \theta_{1} d \phi_{2} \sin \theta_{2} d \theta_{2} d \boldsymbol{\chi} d \tilde{p}_{\theta_{1}} d \tilde{p}_{\phi_{1}} d \tilde{p}_{\theta_{2}} d \tilde{p}_{\phi_{2}} \\
\sqrt{1-\left(\boldsymbol{u}_{1} \cdot \boldsymbol{u}_{2}\right)^{2}} \exp \left[-\frac{1}{2}\left(\boldsymbol{\chi}^{2}+\tilde{p}_{\phi_{1}}^{2}+\tilde{p}_{\phi_{2}}^{2}+\tilde{p}_{\theta_{1}}^{2}+\tilde{p}_{\theta_{2}}^{2}\right)\right] \times \\
\left|\tilde{\boldsymbol{V}} \cdot \boldsymbol{u}_{\perp}\right| \Theta\left(p\left|\tilde{\boldsymbol{V}} \cdot \boldsymbol{u}_{\perp}\right|\right) \Theta(|a|-L / 2) \Theta(|b|-L / 2) \Delta E_{12},
\end{gathered}
$$

all expressed in new variables and $\dot{\boldsymbol{u}}$ by eq. (A.18), e.g.

$$
\tilde{\boldsymbol{V}}=\sqrt{2 T_{\mathrm{trans}} / m} \boldsymbol{\chi}-a \sqrt{T_{\mathrm{rot}} / I}\left(\tilde{p}_{\theta_{1}} \boldsymbol{e}_{\theta_{1}}+\tilde{p}_{\phi_{1}} \boldsymbol{e}_{\phi_{1}}\right)-b \sqrt{T_{\mathrm{rot}} / I}\left(\tilde{p}_{\theta_{2}} \boldsymbol{e}_{\theta_{2}}+\tilde{p}_{\phi_{2}} \boldsymbol{e}_{\phi_{2}}\right) .
$$

\footnotetext{
${ }^{1}$ This reflects the fact that if one particle touches the other from 'above' the sign of the relative velocity of the contact point has to be negative if the particle touches from 'below' the velocity has to be positive.
} 
We want to perform the remaining Gaussian integrals, but we have expressed different terms either in $\left(\boldsymbol{u}_{i}^{\perp}, \boldsymbol{u}^{\perp}\right)$ defined according to eq. (2.17) with $i=1,2$, or in $\left(\boldsymbol{e}_{\theta_{i}}, \boldsymbol{e}_{\phi_{i}}\right)$. It is useful to note that $\left(\boldsymbol{u}_{i}^{\perp}, \boldsymbol{u}^{\perp}\right)$ and $\left(\boldsymbol{e}_{\theta_{i}}, \boldsymbol{e}_{\phi_{i}}\right)$ are two different orthonormal basis of the plane perpendicular to $\boldsymbol{u}_{i}$, so that we can make an orthogonal coordinate transformation from one system to the other. The variables $\tilde{p}_{\theta_{i}}$ and $\tilde{p}_{\phi_{1}}$ are now standard normally distributed and after an orthogonal coordinate transformation the new coordinates will again be standard normally distributed. This means we can equivalently write $\left(\tilde{p}_{\theta_{i}} \boldsymbol{e}_{\theta_{i}}+\tilde{p}_{\phi_{i}} \boldsymbol{e}_{\phi_{i}}\right)$ or $\left(v_{i} \boldsymbol{u}_{i}^{\perp}+w_{i} \boldsymbol{u}^{\perp}\right)$ with standard normally distributed variables $v_{i}$ and $w_{i}$. With this definition of $v_{i}$ and $w_{i}$ we are able to evaluate, for example, terms of the form $\left(\tilde{p}_{\theta_{1}} \boldsymbol{e}_{\theta_{1}}+\tilde{p}_{\phi_{1}} \boldsymbol{e}_{\phi_{1}}\right) \cdot \boldsymbol{u}_{\perp} \equiv\left(v_{1} \boldsymbol{u}_{1}^{\perp}+w_{1} \boldsymbol{u}_{\perp}\right) \cdot \boldsymbol{u}_{\perp}=$ $w_{1}$, where we used $\boldsymbol{u}_{1}^{\perp} \cdot \boldsymbol{u}^{\perp}=\boldsymbol{u}_{2}^{\perp} \cdot \boldsymbol{u}^{\perp}=0$. We can integrate freely over $v_{1}$ and $v_{2}$ and the two components of $\chi$ perpendicular to $\boldsymbol{u}_{\perp}$. We denote with $d \Omega_{i}:=d \phi_{i} \sin \left(\theta_{i}\right) d \theta_{i}$ and the intermediate result reads

$$
\begin{aligned}
\sum_{p= \pm 1} \frac{N-1}{2 V} \frac{1}{(4 \pi)^{2}} \frac{1}{(2 \pi)^{(3 / 2)}} \int d a d b d \boldsymbol{s} d \Omega_{1} d \Omega_{2} \exp \left(-\frac{1}{2} \boldsymbol{s}^{2}\right) \sqrt{1-\left(\boldsymbol{u}_{1} \cdot \boldsymbol{u}_{2}\right)^{2}} \times \\
|\boldsymbol{G} \cdot \boldsymbol{s}| \Theta(p \boldsymbol{G} \cdot \boldsymbol{s})\left[-s_{1} \sqrt{\frac{2 T_{\mathrm{tr}}}{m}} \frac{1+e_{n}}{2} \frac{1}{\frac{1}{m}+\frac{a^{2}}{2 I}+\frac{b^{2}}{2 I}} \boldsymbol{G} \cdot \boldsymbol{s}+\right. \\
\left.\frac{1}{m}\left(\frac{1+e_{n}}{2}\right)^{2}\left(\frac{1}{\frac{1}{m}+\frac{a^{2}}{2 I}+\frac{b^{2}}{2 I}}\right)^{2}(\boldsymbol{G} \cdot \boldsymbol{s})^{2}\right] .
\end{aligned}
$$

We introduced the vectors

$$
\begin{aligned}
\boldsymbol{s} & :=\left(s_{1}, s_{2}, s_{3}\right):=\left(\boldsymbol{\chi} \cdot \boldsymbol{u}_{\perp}, w_{1}, w_{2}\right) \text { and } \\
\boldsymbol{G} & =\left(\sqrt{\frac{2 T_{\mathrm{tr}}}{m}},-a \sqrt{\frac{T_{\mathrm{rot}}}{I}},-b \sqrt{\frac{T_{\mathrm{rot}}}{I}}\right) .
\end{aligned}
$$

We can perform the integral over $\boldsymbol{s}$. We sketch here only how this is done. We want to integrate

$$
\int d \boldsymbol{s} \exp \left(-\frac{1}{2} \boldsymbol{s}^{2}\right) \Theta( \pm \boldsymbol{G} \cdot \boldsymbol{s})|\boldsymbol{G} \cdot \boldsymbol{s}|(\boldsymbol{G} \cdot \boldsymbol{s}) s_{1}
$$

Let $\left(\boldsymbol{e}_{1}, \boldsymbol{e}_{2}, \boldsymbol{e}_{3}\right)$ be the original coordinate system and we define a coordinate system $\left(\boldsymbol{e}_{x}, \boldsymbol{e}_{y}, \boldsymbol{e}_{z}\right)$ in which the $z$-axis is parallel to $\boldsymbol{G}$ and we decompose $\boldsymbol{s}$ in this coordinate system $s=\left(s_{x}, s_{y}, s_{z}\right)$. Then eq. (A.29) reads

$$
\begin{aligned}
& \int d s_{x} d s_{y} d s_{z} \exp \left(-\frac{1}{2}\left(s_{x}^{2}+s_{y}^{2}+s_{z}^{2}\right)\right) \Theta\left( \pm s_{z}\right) \times \\
& |\boldsymbol{G}|\left|s_{z}\right||\boldsymbol{G}| s_{z}\left[\left(s_{x} \boldsymbol{e}_{x}+s_{y} \boldsymbol{e}_{y}+s_{z} \boldsymbol{e}_{z}\right) \cdot \boldsymbol{e}_{1}\right]
\end{aligned}
$$




\section{A. Appendix}

Only the term, which is proportional to $s_{z} \boldsymbol{e}_{z}$, contributes and the Gaussian integral can easily be performed. Using that $|\boldsymbol{G}| \boldsymbol{e}_{z}=\boldsymbol{G}$ we write $|\boldsymbol{G}| \boldsymbol{e}_{z} \cdot \boldsymbol{e}_{1}=\boldsymbol{G} \cdot \boldsymbol{e}_{1}=G_{1}$ and we end up with the result $4 \pi|\boldsymbol{G}| G_{1}$. Only the integrals over $\Omega_{1}$ and $\Omega_{2}$ have to be done with standard techniques. All other integrals are performed similarly and the results are quoted in the main text. 


\section{A.3. Generalized Laguerre polynomials}

The generalized Laguerre polynomials $L_{n}^{(\alpha)}$, for $\alpha>-1$, are the orthogonal polynomials associated with the interval $(0, \infty)$, the weight function $w(x)=x^{\alpha} e^{-x}$, and standardized by the condition that the coefficient of $x^{n}$ in $L_{n}^{(\alpha)}(x)$ equals $\frac{(-1)^{n}}{n !}$.

Examples:

$$
\begin{aligned}
& L_{0}^{(\alpha)}(x)=1 \\
& L_{1}^{(\alpha)}(x)=\alpha+1-x \\
& L_{2}^{(\alpha)}(x)=\frac{1}{2}\left[(\alpha+1)(\alpha+2)-2(\alpha+2) x+x^{2}\right]
\end{aligned}
$$

Orthogonality Relation:

$$
\int_{0}^{\infty} e^{-x} x^{\alpha} L_{n}^{(\alpha)}(x) L_{m}^{(\alpha)}(x) d x=\delta_{m, n} \Gamma(1+\alpha)\left(\begin{array}{c}
n+\alpha \\
n
\end{array}\right)
$$

Differentiation formula:

$$
\frac{d}{d x} L_{n}^{(\alpha)}(x)=\frac{n L_{n}^{(\alpha)}(x)-(n+\alpha) L_{n-1}^{(\alpha)}(x)}{x}
$$

\section{Laguerre polynomials in $D$-dimensions}

By choosing $\alpha$ for the Laguerre Polynomials $L_{n}^{(\alpha)}(x)$ equal $\alpha=D / 2-1$ we get

$$
\begin{gathered}
\pi^{-D / 2} \int_{-\infty}^{\infty} d \boldsymbol{v} \exp \left(-\boldsymbol{v}^{2}\right) L_{n}^{(\alpha)}\left(\boldsymbol{v}^{2}\right) L_{m}^{(\alpha)}\left(\boldsymbol{v}^{2}\right)= \\
\pi^{-D / 2} S_{D} \int_{0}^{\infty} d v v^{d-1} \exp \left(-v^{2}\right) L_{n}^{(\alpha)}\left(v^{2}\right) L_{m}^{(\alpha)}\left(v^{2}\right)= \\
\pi^{-D / 2} S_{D} \int_{0}^{\infty} d c \frac{1}{2} c^{\alpha} \exp (-c) L_{n}^{(\alpha)}(c) L_{m}^{(\alpha)}(c)
\end{gathered}
$$

$S_{D}=\frac{2 \pi^{D / 2}}{\Gamma(D / 2)}$ is the surface area of a sphere in $D$ dimensions, and together with the orthogonality relation of eq. (A.34) we finally have

$$
\frac{1}{\pi^{D / 2}} \int_{-\infty}^{\infty} d \boldsymbol{v} \exp \left(-\boldsymbol{v}^{2}\right) L_{n}^{(\alpha)}\left(\boldsymbol{v}^{2}\right) L_{m}^{(\alpha)}\left(\boldsymbol{v}^{2}\right)=\delta_{m, n}\left(\begin{array}{c}
n+\alpha \\
n
\end{array}\right)
$$




\section{A.4. The time dependence of the moments 1}

In section 3.2.1 we determined the $N$-particle distribution function in eq. (3.32) as

$$
\begin{aligned}
\rho(\Gamma, t)=\frac{1}{Q^{N}} W\left(\boldsymbol{r}_{1}, \ldots, \boldsymbol{r}_{n}\right) \prod_{i=1}^{N} \rho_{i}\left(\boldsymbol{v}_{i}, t\right), \text { and } & \\
\rho_{i}\left(\boldsymbol{v}_{i}, t\right):= & \frac{1}{\left(v_{0}(t) \sqrt{\pi}\right)^{D}} \tilde{\rho}_{i}\left(\boldsymbol{c}_{i}, t\right):= \\
& \frac{1}{\left(v_{0}(t) \sqrt{\pi}\right)^{D}} \exp \left(-\boldsymbol{c}_{i}^{2}\right) \sum_{n=0}^{\infty} a_{n}(t) L_{n}^{\alpha}\left(\boldsymbol{c}_{i}^{2}\right) .
\end{aligned}
$$

$Q$ is chosen so that $\int \prod_{i} d \boldsymbol{r}_{i} W=1$ and we have expressed $\rho_{i}$ by a scaling form $\tilde{\rho}_{i}\left(\boldsymbol{c}_{i}, t\right) . \rho_{i}$ is normalized $\int d \boldsymbol{v}_{i} \rho_{i}=1$ and it holds with eqs. (A.32)(A.33) that

$$
\boldsymbol{v}^{2}=-L_{1}^{\alpha}\left(\boldsymbol{v}^{2}\right)-(\alpha+1) L_{0}^{\alpha}\left(\boldsymbol{v}^{2}\right) .
$$

Using the orthogonality relations eq. (A.37) we find that

$$
\int d \boldsymbol{v}_{1} \rho_{1}(\boldsymbol{v}, t) \boldsymbol{v}^{2}=v_{0}^{2}\left(\frac{D}{2}-\left(\begin{array}{c}
1+\alpha \\
1
\end{array}\right) a_{1}\right)
$$

so that we have to choose $a_{1}=0$ for all times.

We compute using eq. (A.15)

$$
\begin{aligned}
& \frac{d}{d t} T=\frac{d}{d t} \frac{m}{2} v_{0}^{2}= m v_{0} \frac{d}{d t} v_{0}=\frac{d}{d t} \frac{m}{D} \int d \Gamma \rho \boldsymbol{v}_{1}^{2}=\frac{1}{N} \frac{m}{D} \frac{d}{d t} \int d \Gamma \rho \sum_{i} \boldsymbol{v}_{i}^{2}= \\
& \frac{m}{D} d^{D-1} \frac{N}{V} g(d) \int d \boldsymbol{v}_{1} d \boldsymbol{v}_{2} d \hat{\boldsymbol{r}} \rho_{1} \rho_{2}\left|\boldsymbol{v}_{12} \hat{\boldsymbol{r}}\right| \Theta\left(-\boldsymbol{v}_{12} \hat{\boldsymbol{r}}\right)(b-1) \boldsymbol{v}_{1}^{2}
\end{aligned}
$$

All velocities are scaled with $v_{0}$ and we make a coordinate transformation to $\boldsymbol{c}=\boldsymbol{v} / v_{0}$.

$$
\begin{aligned}
\frac{d}{d t} T & = \\
\frac{m}{D} d^{D-1} \frac{N}{V} g(d) v_{0}^{3} \frac{1}{\pi^{D}} \int d \boldsymbol{c}_{1} d \boldsymbol{c}_{2} d \hat{\boldsymbol{r}} \tilde{\rho}_{1}\left(\boldsymbol{c}_{1}\right) \tilde{\rho}_{2}\left(\boldsymbol{c}_{2}\right)\left|\boldsymbol{c}_{12} \hat{\boldsymbol{r}}\right| \Theta\left(-\boldsymbol{c}_{12} \hat{\boldsymbol{r}}\right)(b-1) \boldsymbol{c}_{1}^{2}=: & -2 \gamma \omega_{0} T .
\end{aligned}
$$

$\omega_{0}$ is the Enskog collision frequency and is quoted in the main text as well as $\gamma$.

We now calculate the time dependence of $a_{l}$ represented by eq. (3.35)

$$
\frac{d}{d t} a_{n}=\frac{1}{\left(\begin{array}{c}
n+\alpha \\
n
\end{array}\right)} \frac{d}{d t} \int d \Gamma \rho L_{n}^{\alpha}\left(\left(\boldsymbol{v}_{1} / v_{0}\right)^{2}\right)
$$

We have to take 1) the time derivative of the distribution function given by operator for the distribution function, and 2) the time derivative of $L_{n}^{\alpha}\left(\left(\boldsymbol{v}_{1} / v_{0}\right)^{2}\right)$ which depends on time via $v_{0}$. 
1. We have to calculate

$$
\begin{aligned}
& \int d \Gamma i \overline{\mathcal{L}} \rho(t) L_{n}^{\alpha}\left(\left(\boldsymbol{v}_{1} / v_{0}\right)^{2}\right)= \\
& \int d \Gamma \rho(t) i \mathcal{L} L_{n}^{\alpha}\left(\left(\boldsymbol{v}_{1} / v_{0}\right)^{2}\right)=\frac{N}{V} d^{D-1} g(d) \int d \boldsymbol{v}_{1} d \boldsymbol{v}_{2} d \hat{\boldsymbol{r}} \\
& \rho_{1} \rho_{2}\left|\boldsymbol{v}_{12} \hat{\boldsymbol{r}}\right| \Theta\left(-\boldsymbol{v}_{12} \hat{\boldsymbol{r}}\right)(b-1) L_{n}^{\alpha}\left(\left(\boldsymbol{v}_{1} / v_{0}\right)^{2}\right) .
\end{aligned}
$$

Again we used eq. (A.15), and we transform to the scaled velocities to get

$$
\frac{1}{\left(\begin{array}{c}
n+\alpha \\
\alpha
\end{array}\right)} \frac{N}{V} d^{D-1} g(d) v_{0} \int d \boldsymbol{V}_{1} d \boldsymbol{V}_{2} d \hat{\boldsymbol{r}} \tilde{\rho}_{1} \tilde{\rho}_{2}\left|\boldsymbol{c}_{12} \hat{\boldsymbol{r}}\right| \Theta\left(-\boldsymbol{c}_{12} \hat{\boldsymbol{r}}\right)(\tilde{b}-1) L_{n}^{\alpha}\left(\boldsymbol{c}_{1}^{2}\right)=: \omega_{0} \gamma_{n} .
$$

2. We want to determine

$$
\int d \Gamma \rho\left(\frac{d}{d t} L_{n}^{\alpha}\left(\left(\boldsymbol{v}_{1} / v_{0}\right)^{2}\right)\right)
$$

We calculate using the differentiation formula eq. (A.35)

$$
\left(\frac{d}{d t} L_{n}^{\alpha}\left(\left(\boldsymbol{v}_{1} / v_{0}\right)^{2}\right)\right)=-2 \frac{\dot{v}_{0}}{v_{0}}\left(n L_{n}^{\alpha}\left(\left(\boldsymbol{v}_{1} / v_{0}\right)^{2}\right)-(n+\alpha) L_{n-1}^{\alpha}\left(\left(\boldsymbol{v}_{1} / v_{0}\right)^{2}\right)\right)
$$

and using the orthogonality relations eq. (A.37) we get

$$
B=-2 \frac{\dot{v}_{0}}{v_{0}}\left(\begin{array}{c}
n+\alpha \\
n
\end{array}\right)\left(n a_{n}-n a_{n-1}\right) .
$$

Collecting terms we find

$$
\frac{d}{d t} a_{n}=\omega_{0}\left(\gamma_{n}+2 n \gamma \omega_{0}\left(a_{n}-a_{n-1}\right)\right.
$$

i.e. eq. (3.39) and eq. (3.40). 


\section{A.5. The time dependence of the moments 2}

We assume that the $N$-particle distribution function is given by eqs. $(3.56,3.57)$ so that it reads

$$
\rho=\frac{1}{Q^{N}} W\left(\boldsymbol{r}_{1}, \ldots, \boldsymbol{r}_{N}\right) \prod_{i=1}^{N} \rho_{i}\left(\boldsymbol{v}_{i}, \boldsymbol{\omega}_{\boldsymbol{i}}\right) .
$$

$Q$ is chosen so that $\int \prod_{i} d \boldsymbol{r}_{i} W=1$ and we only need that $\rho_{i}$ can be written in a scaling form $\rho_{i}=\frac{1}{v_{0}^{D_{\text {tr }}}} \frac{1}{\omega_{0}^{D_{\text {rot }}}} \tilde{\rho}_{i}\left(\boldsymbol{v}_{i} / v_{0}, \boldsymbol{\omega}_{\boldsymbol{i}} / \omega_{0}\right)$ with average (angular) velocity $v_{0}\left(\omega_{0}\right)$. This is consistent with eq. (3.56). Using eq. (A.15) we compute

$$
\begin{aligned}
\frac{d}{d t} \frac{D_{\mathrm{tr}}}{2} v_{0}^{2}=D_{\mathrm{tr}} v_{0} \frac{d}{d t} v_{0}=\frac{d}{d t} \int d \Gamma \rho \boldsymbol{v}_{1}^{2}=\frac{1}{N} \frac{d}{d t} \int d \Gamma \rho \sum_{i} \boldsymbol{v}_{i}^{2}= \\
d^{D_{\mathrm{tr}}-1} \frac{N}{V} g(d) \int d \boldsymbol{\omega}_{1} d \boldsymbol{\omega}_{2} d \boldsymbol{v}_{1} d \boldsymbol{v}_{2} d \hat{\boldsymbol{r}} \rho_{1} \rho_{2}\left|\boldsymbol{v}_{12} \hat{\boldsymbol{r}}\right| \Theta\left(-\boldsymbol{v}_{12} \hat{\boldsymbol{r}}\right)(b-1) \boldsymbol{v}_{1}^{2} .
\end{aligned}
$$

We scale all (angular) velocities with $v_{0}\left(\omega_{0}\right)$ and introduce the dimensionless variables $\boldsymbol{V}_{i}=\frac{\boldsymbol{v}_{i}}{v_{0}}$ and $\boldsymbol{\Omega}_{i}=\frac{\boldsymbol{\omega}_{i}}{\omega_{0}}$ and insert the scaling form and end up with

$$
\begin{aligned}
& \frac{d}{d t} \frac{D_{\mathrm{tr}}}{2} v_{0}^{2}=d^{D_{\mathrm{tr}}-1} \frac{N}{V} g(d) v_{0}^{3} \int d \boldsymbol{\Omega}_{1} d \boldsymbol{\Omega}_{2} d \boldsymbol{V}_{1} d \boldsymbol{V}_{2} d \hat{\boldsymbol{r}} \\
& \tilde{\rho}_{1} \tilde{\rho}_{2}\left|\boldsymbol{V}_{12} \hat{\boldsymbol{r}}\right| \Theta\left(-\boldsymbol{V}_{12} \hat{\boldsymbol{r}}\right)(\tilde{b}-1) \boldsymbol{V}_{1}^{2}=: d^{D_{\mathrm{tr}}-1} \frac{N}{V} g(d) v_{0}^{3} \mu_{2}^{0} .
\end{aligned}
$$

$\tilde{b}$ is the operator which changes scaled variables from pre- to post-collisional values and is discussed at the end of this section. Analogously we calculate the time dependence of $\omega_{0}$ to get

$$
\begin{aligned}
\frac{d}{d t} \frac{D_{\mathrm{rot}}}{2} \omega_{0}^{2}=d^{D_{\mathrm{tr}}-1} & \frac{N}{V} g(d) v_{0} \omega_{0}^{2} \int d \boldsymbol{\Omega}_{1} d \boldsymbol{\Omega}_{2} d \boldsymbol{V}_{1} d \boldsymbol{V}_{2} d \hat{\boldsymbol{r}} \\
& \tilde{\rho}_{1} \tilde{\rho}_{2}\left|\boldsymbol{V}_{12} \hat{\boldsymbol{r}}\right| \Theta\left(-\boldsymbol{V}_{12} \hat{\boldsymbol{r}}\right)(\tilde{b}-1) \boldsymbol{\Omega}_{1}^{2}=: d^{D_{\mathrm{tr}}-1} \frac{N}{V} g(d) v_{0} \omega_{0}^{2} \mu_{0}^{2} .
\end{aligned}
$$

We now calculate the time dependence of $a_{m, n}$ represented by eq. (3.58):

$$
\begin{aligned}
\frac{d}{d t} a_{n, m}=\frac{1}{\left(\begin{array}{c}
n+\alpha \\
n
\end{array}\right)\left(\begin{array}{c}
m+\beta \\
m
\end{array}\right)} \frac{d}{d t} \int d \Gamma \rho L_{n}^{\alpha}\left(\left(\boldsymbol{v}_{1} / v_{0}\right)^{2}\right) L_{m}^{\beta}\left(\left(\boldsymbol{\omega}_{1} / \omega_{0}\right)^{2}\right)=: & \frac{1}{\left(\begin{array}{c}
n+\alpha \\
n
\end{array}\right)\left(\begin{array}{c}
m+\beta \\
m
\end{array}\right)}(A+B+C) .
\end{aligned}
$$

The term $A$ stems from the time derivative of the distribution function, $B$ from the time derivative of $L_{n}^{\alpha}\left(\left(\boldsymbol{v}_{1} / v_{0}\right)^{2}\right)$ which depends on time via $v_{0}$ and $C$ from the time 
derivative of $L_{m}^{\beta}\left(\left(\boldsymbol{\omega}_{1} / \omega_{0}\right)^{2}\right)$. First we calculate term $A$ :

\section{Term A}

$$
\begin{gathered}
A=\int d \Gamma i \overline{\mathcal{L}} \rho(t) L_{n}^{\alpha}\left(\left(\boldsymbol{v}_{1} / v_{0}\right)^{2}\right) L_{m}^{\beta}\left(\left(\boldsymbol{\omega}_{1} / \omega_{0}\right)^{2}\right)= \\
\int d \Gamma \rho(t) i \mathcal{L} L_{n}^{\alpha}\left(\left(\boldsymbol{v}_{1} / v_{0}\right)^{2}\right) L_{m}^{\beta}\left(\left(\boldsymbol{\omega}_{1} / \omega_{0}\right)^{2}\right)= \\
\int d \boldsymbol{\omega}_{1} d \boldsymbol{\omega}_{2} d \boldsymbol{v}_{1} d \boldsymbol{v}_{2} d \hat{\boldsymbol{r}} \rho_{1} \rho_{2}\left|\boldsymbol{v}_{12} \hat{\boldsymbol{r}}\right| \Theta\left(-\boldsymbol{v}_{12} \hat{\boldsymbol{r}}\right)(b-1) \times \\
L_{n}^{\alpha}\left(\left(\boldsymbol{v}_{1} / v_{0}\right)^{2}\right) L_{m}^{\beta}\left(\left(\boldsymbol{\omega}_{1} / \omega_{0}\right)^{2}\right) .
\end{gathered}
$$

Again we used eq. (A.15), and we transform to the scaled velocities and obtain

$$
\begin{aligned}
A=\frac{N}{V} d^{D_{\mathrm{tr}}-1} g(d) v_{0} \int d \boldsymbol{\Omega}_{1} d \boldsymbol{\Omega}_{2} d \boldsymbol{V}_{1} d \boldsymbol{V}_{2} d \hat{\boldsymbol{r}} & \\
\tilde{\rho}_{1} \tilde{\rho}_{2}\left|\boldsymbol{V}_{12} \hat{\boldsymbol{r}}\right| \Theta\left(-\boldsymbol{V}_{12} \hat{\boldsymbol{r}}\right)(\tilde{b}-1) L_{n}^{\alpha}\left(\boldsymbol{V}_{1}^{2}\right) L_{m}^{\beta}\left(\boldsymbol{\Omega}_{1}^{2}\right)=: & \frac{N}{V} d^{D_{\mathrm{tr}}-1} g(d) v_{0} \lambda_{n}^{m}
\end{aligned}
$$

Next we calculate the terms $B$ and $C$ :

\section{Term B}

$$
B=\int d \Gamma \rho\left(\frac{d}{d t} L_{n}^{\alpha}\left(\left(\boldsymbol{v}_{1} / v_{0}\right)^{2}\right)\right) L_{m}^{\beta}\left(\left(\boldsymbol{\omega}_{1} / \omega_{0}\right)^{2}\right)
$$

We calculate using the differentiation formula eq. (A.35)

$$
\left(\frac{d}{d t} L_{n}^{\alpha}\left(\left(\boldsymbol{v}_{1} / v_{0}\right)^{2}\right)\right)=-2 \frac{\dot{v}_{0}}{v_{0}}\left(n L_{n}^{\alpha}\left(\left(\boldsymbol{v}_{1} / v_{0}\right)^{2}\right)-(n+\alpha) L_{n-1}^{\alpha}\left(\left(\boldsymbol{v}_{1} / v_{0}\right)^{2}\right)\right)
$$

and using the orthogonality relations eq. (A.37) we get

$$
B=-2 \frac{\dot{v}_{0}}{v_{0}}\left(\begin{array}{c}
n+\alpha \\
n
\end{array}\right)\left(\begin{array}{c}
m+\beta \\
m
\end{array}\right)\left(n a_{n, m}-n a_{n-1, m}\right)
$$

Similarly we get

\section{Term C}

$$
C=-2 \frac{\dot{\omega}_{0}}{\omega_{0}}\left(\begin{array}{c}
n+\alpha \\
n
\end{array}\right)\left(\begin{array}{c}
m+\beta \\
m
\end{array}\right)\left(m a_{n, m}-m a_{n, m-1}\right)
$$

Combining $A, B, C$ in eq. (A.55), replacing $\dot{v}_{0}$ by eq. (A.52) and $\dot{\omega}_{0}$ by eq. (A.53) we get

$$
\begin{aligned}
& \frac{d}{d t} a_{n, m}=d^{D_{\operatorname{tr}}-1} \frac{N}{V} g(d) v_{0} \times \\
& \quad\left[\frac{\lambda_{n}^{m}}{\left(\begin{array}{c}
n+\alpha \\
n
\end{array}\right)\left(\begin{array}{c}
m+\beta \\
m
\end{array}\right)}-2 \mu_{2}^{0} \frac{1}{D_{\mathrm{tr}}} n\left[a_{n, m}-a_{n-1, m}\right]-2 \mu_{0}^{2} \frac{1}{D_{\mathrm{rot}}} m\left[a_{n, m}-a_{n, m-1}\right]\right]
\end{aligned}
$$


In the asymptotic state we assume that that the ratio $v_{0} / \omega_{0}$ is constant in time and so are all $a_{n, m}$. To determine the ratio $v_{0} / \omega_{0}$ we divide eq. (A.52) by eq. (A.53) and get

$$
\frac{D_{\mathrm{tr}} \dot{v}_{0}}{D_{\mathrm{rot}} \dot{\omega}_{0}}=\frac{v_{0} \mu_{2}^{0}}{\omega_{0} \mu_{0}^{2}}
$$

Constant ratio $v_{0} / \omega_{0}$ implies that $\frac{\dot{v}_{0}}{\dot{\omega}_{0}}=\frac{v_{0}}{\omega_{0}}$ and we get the following equation to determine the ratio of translational to translational velocity

$$
\frac{D_{\mathrm{tr}}}{D_{\mathrm{rot}}} \mu_{0}^{2}=\mu_{2}^{0}
$$

Time independence of all $a_{n, m}$ together with eq. (A.63) can be written as:

$$
\lambda_{n}^{m}=\frac{2}{D_{\mathrm{tr}}} \mu_{2}^{0}\left(\begin{array}{c}
n+\alpha \\
n
\end{array}\right)\left(\begin{array}{c}
m+\beta \\
m
\end{array}\right)\left[(n+m) a_{n, m}-n a_{n-1, m}-m a_{n, m-1}\right] .
$$

Eqs. (A.63,A.64) represent an infinitely large non-linear set of equations, which can be solved only approximately.

For completeness reasons we give the collision rules for dimensionless quantities, which can be written by introducing the dimensionless ratio $K_{+}=\frac{m}{I} \frac{v_{0}^{2}}{\omega_{0}^{2}}$ as in eq. (3.17)

$$
\begin{aligned}
& b \boldsymbol{V}_{1}=\boldsymbol{V}_{1}^{\prime}=\boldsymbol{V}_{1}-\eta_{t} \boldsymbol{V}_{12}-\left(\eta_{n}-\eta_{t}\right)\left(\hat{\boldsymbol{r}} \cdot \boldsymbol{V}_{12}\right) \hat{\boldsymbol{r}}-\eta_{t} \frac{1}{\sqrt{q K_{+}}} \hat{\boldsymbol{r}} \times\left(\boldsymbol{\Omega}_{1}+\boldsymbol{\Omega}_{2}\right), \\
& b \boldsymbol{V}_{2}=\boldsymbol{V}_{2}^{\prime}=\boldsymbol{V}_{2}+\eta_{t} \boldsymbol{V}_{12}+\left(\eta_{n}-\eta_{t}\right)\left(\hat{\boldsymbol{r}} \cdot \boldsymbol{V}_{12}\right) \hat{\boldsymbol{r}}+\eta_{t} \frac{1}{\sqrt{q K_{+}}} \hat{\boldsymbol{r}} \times\left(\boldsymbol{\Omega}_{1}+\boldsymbol{\Omega}_{2}\right), \\
& b \boldsymbol{\Omega}_{1}=\boldsymbol{\Omega}_{1}^{\prime}=\boldsymbol{\Omega}_{1}+\frac{\sqrt{K_{+}}}{\sqrt{q}} \eta_{t} \hat{\boldsymbol{r}} \times \boldsymbol{V}_{12}+\frac{\eta_{t}}{q} \hat{\boldsymbol{r}} \times\left(\hat{\boldsymbol{r}} \times\left(\boldsymbol{\Omega}_{1}+\boldsymbol{\Omega}_{2}\right)\right), \\
& b \boldsymbol{\Omega}_{2}=\boldsymbol{\Omega}_{2}^{\prime}=\boldsymbol{\Omega}_{2}+\frac{\sqrt{K_{+}}}{\sqrt{q}} \eta_{t} \hat{\boldsymbol{r}} \times \boldsymbol{V}_{12}+\frac{\eta_{t}}{q} \hat{\boldsymbol{r}} \times\left(\hat{\boldsymbol{r}} \times\left(\boldsymbol{\Omega}_{1}+\boldsymbol{\Omega}_{2}\right)\right) .
\end{aligned}
$$




\section{A.6. Collision integrals with MAPLE}

In this appendix we give some hints how to improve the performance of a computer algebra program like MAPLE to calculate collision integral for spheres. We want to calculate integrals of the form given in eq. (A.15)

$$
\int d \boldsymbol{\omega}_{1} d \boldsymbol{\omega}_{2} d \boldsymbol{v}_{1} d \boldsymbol{v}_{2} d \hat{\boldsymbol{r}} \rho_{1} \rho_{2}\left|\boldsymbol{v}_{12} \hat{\boldsymbol{r}}\right| \Theta\left(-\boldsymbol{v}_{12} \hat{\boldsymbol{r}}\right)(b-1) \frac{1}{2}\left(f_{1}+f_{2}\right)
$$

$(b-1)\left(f_{1}+f_{2}\right)$ are multinomials in the components of the vectors $\boldsymbol{v}_{1}, \boldsymbol{v}_{2}, \boldsymbol{\omega}_{1}, \boldsymbol{\omega}_{2}$ determined by the collisions rules and $\hat{\boldsymbol{r}}$. We assume that $\rho_{i}$ is of the form of a Gaussian multiplied with a multinomial in the vectors $\boldsymbol{v}_{1}, \boldsymbol{v}_{2}, \boldsymbol{\omega}_{1}, \boldsymbol{\omega}_{2}$. For symmetry reasons after integration over all velocities and angular velocities the remaining integral over $\hat{\boldsymbol{r}}$ does not depend on $\hat{\boldsymbol{r}}$. So we can choose it in the direction of let us say $\boldsymbol{e}^{z}$ and first perform the integral over all velocities. Then we multiply the result with the value for the surface of the unit sphere. We are left with a 12-dimensional Gaussian integral (in three dimensions) over a large multinomial, but we have to take into account the $\Theta$-function, which is for the special choice of $\hat{\boldsymbol{r}}$ given by $\Theta\left(-\left(\boldsymbol{v}_{1}^{z}-\boldsymbol{v}_{2}^{z}\right)\right)$. Since the polynomial is large, the build-in integration routine can not handle it. So the idea is the following: We want to integrate one after another over $\boldsymbol{\omega}_{1}^{x}, \boldsymbol{\omega}_{1}^{y}, \ldots, \boldsymbol{v}_{2}^{y}$. To integrate for example over $\boldsymbol{\omega}_{1}^{x}$ we let MAPLE perform a Taylor expansion of the integrand (which is done by this program quite fast and without problems) in $\boldsymbol{\omega}_{1}^{x}$, take the $n$-th coefficients of this Taylor expansion and multiply it with the $n$-th moment of a Gaussian integral. Then we collect all terms together. Only for the last integrations over $\boldsymbol{v}_{1}^{z}$ and $\boldsymbol{v}_{2}^{z}$ we first integrate over $\boldsymbol{v}_{1}^{z}$ up to $\boldsymbol{v}_{2}^{z}$ and then integrate over $\boldsymbol{v}_{2}^{z}$ to take into account the $\Theta$-function.

For the MAPLE code see any textbook about MAPLE, for example [Ko97]. The integral of the integrand integrand over one velocity component $\mathrm{b}$ to $\mathrm{n}$-th order in $\mathrm{b}$ is performed by the following procedure

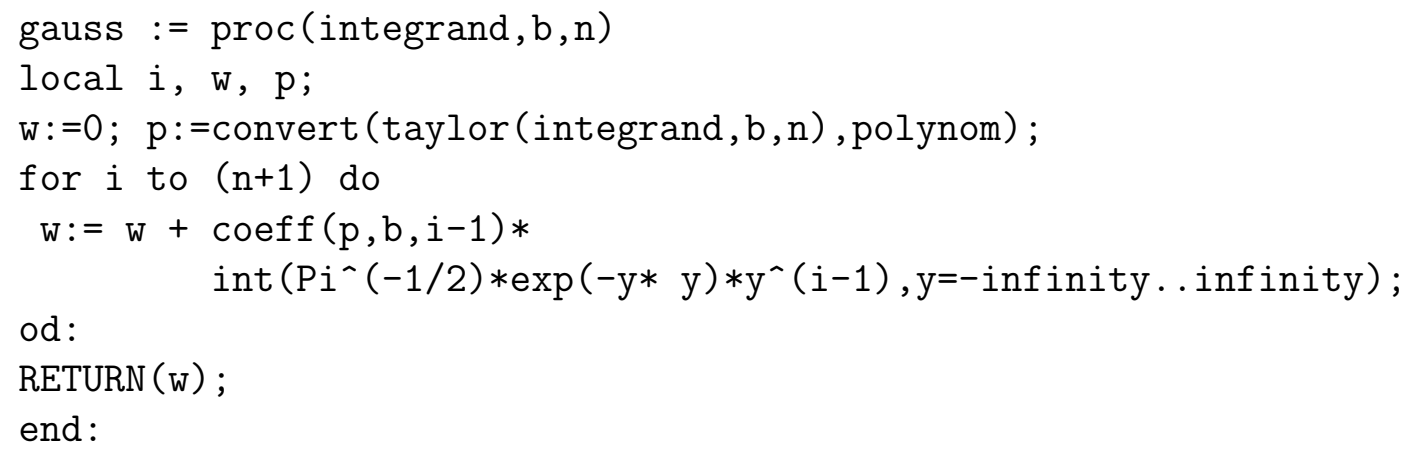

The order $\mathrm{n}$ depends on the order of approximation and can be determined by a simple power counting.

Gaussian integration up to a upper limit ober is performed by: 


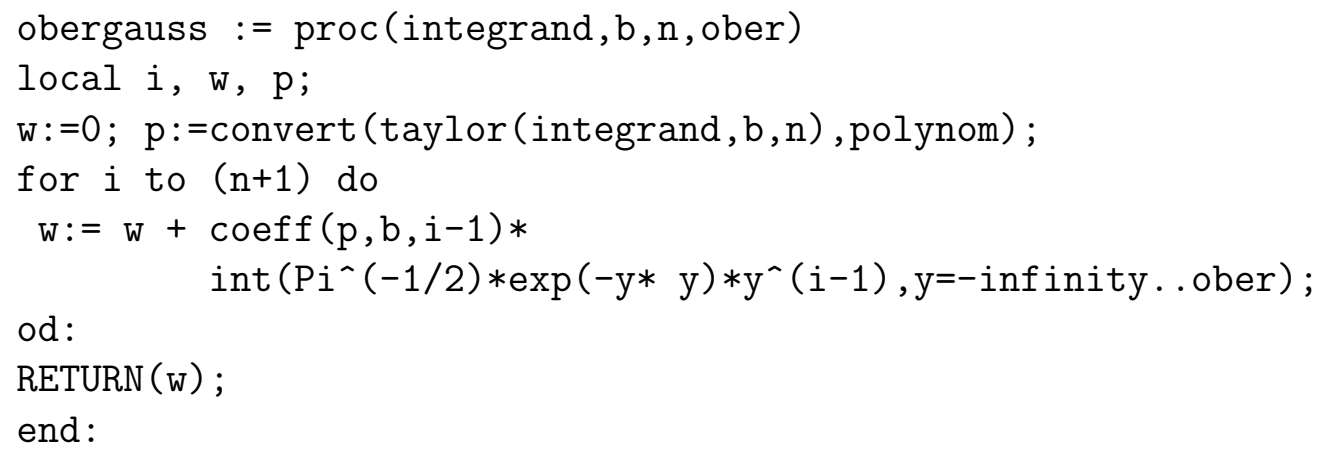

A further trick has to be used to perform the last integral over $\boldsymbol{v}_{2}^{z}$. The integrand is still a large sum of functions in $\boldsymbol{v}_{2}^{z}$, but MAPLE normally tries to integrate over the whole expression, which makes problems for too large calculations. Therefore we integrate over each addend separately which is done by the following procedure:

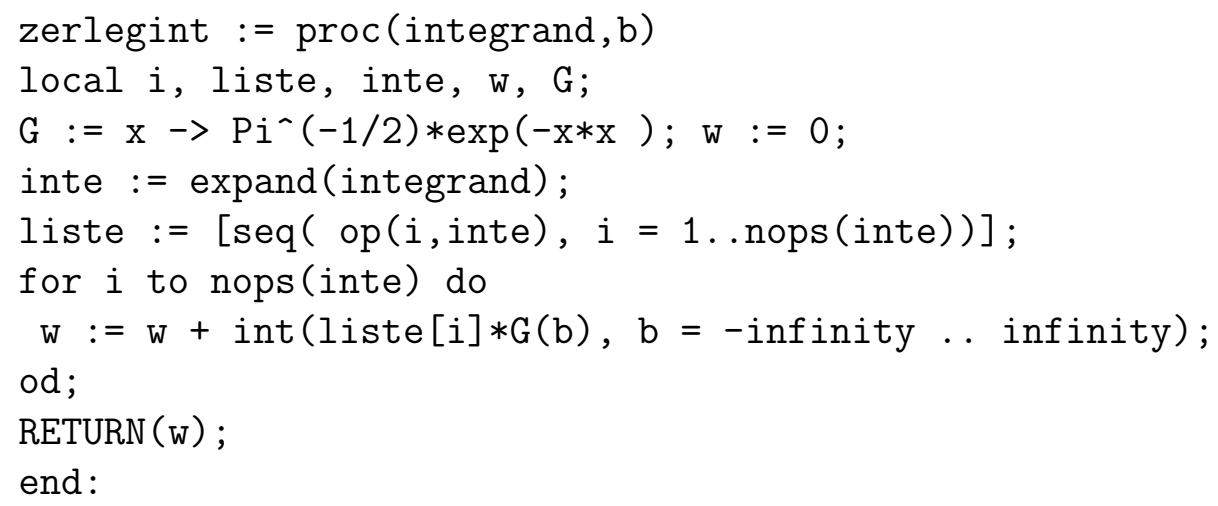

One may ask now, why we have not used the procedure zerlegint instead of the procedure gauss. The reason for that is that MAPLE is not able to perform expand for such a large polynomial, as it exists before all the other integrations are done.

The whole integral over all velocities now (in 3 dimensions) look like:

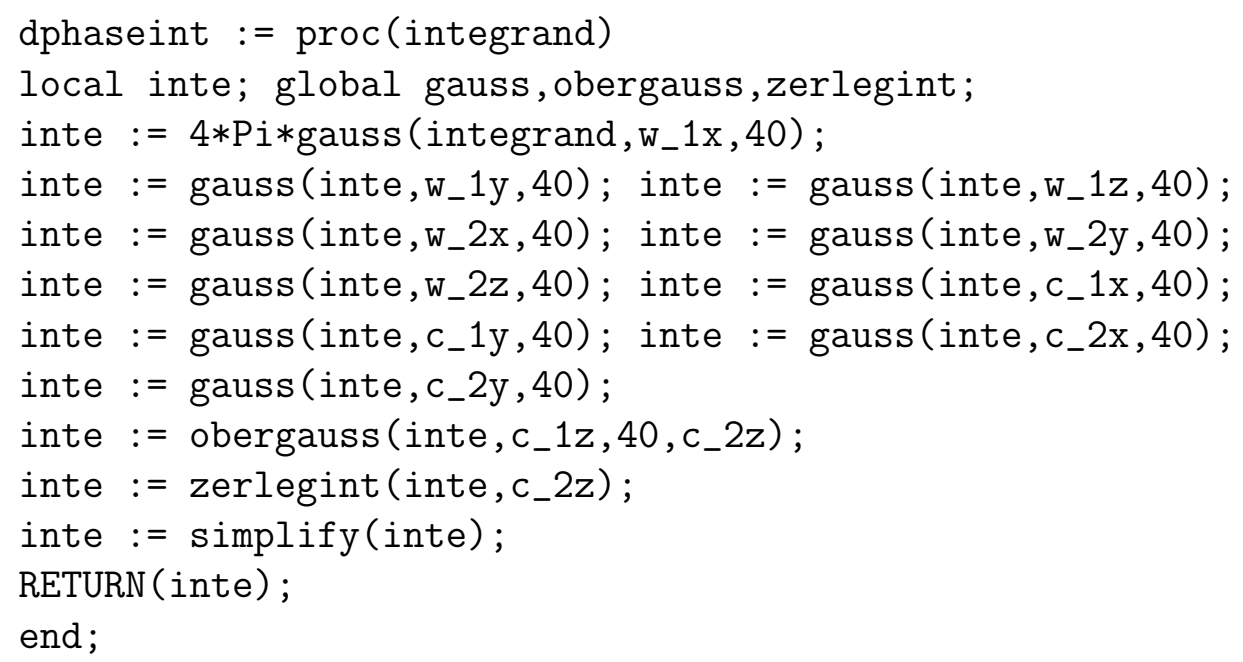




\section{Bibliography}

[AlGa70] B. J. Alder, D. M. Gass and T. E. Wainwright, J. Chem. Phys. 53, 3813 (1970).

[AlTi87] M. P. Allen and D. J. Tildesley, Computer Simulations of Liquids, Clarendon Press, Oxford (1987).

[AlWa62] B. J. Alder and T. E. Wainwright, Phys. Rev. 127, 359 (1962).

[AsGi98] T. Aspelmeier, G. Giese and A. Zippelius, Phys. Rev. E 57, 857 (1998).

[BaBe90] G. W. Baxter and R. P. Behringer, Phys. Rev. A42, 1017 (1990).

[BeEr79] H. van Beijeren and M. H. Ernst, J. Stat. Phys. 21, 125 (1979).

[Bi76] G. A. Bird. Molecular Gas Dynamics Oxford Univ. Press, London (1976).

[Br96] B. Brogliato, Nonsmooth Impact mechanics, Springer (1996).

[BrCu99] J. J. Brey, D. Cubero, and M. J. Ruiz-Montero, Phys. Rev. E 59, 1256 (1999).

[BrDu98] J. J. Brey, J. W. Dufty, C. S. Kim and A. Santos, Phys. Rev. E 584638 , (1998).

[BrEr98] R. Brito and M. H. Ernst, Europhysics Letters, 43, 497 (1998).

[BrEr99] R. Brito and M. H. Ernst, Int. J. Mod. Phys. C, 9, 1339 (1998).

[BrMo96] J. J. Brey, F. Moreno and J. W. Dufty, Phys. Rev. E 54, 445 (1996).

[BrMo98] J. J. Brey, F. Moreno and M. J. Ruiz-Montero, Phys. Fluids 10, 2965 (1998); 2976 (1998).

[BrOr99] R. Brito and J. A. G. Orza, private communication (1999).

[BrPo99] N. V. Brilliantov and T. Pöschel, cond-mat/9906404 (1999). 
[BrRu96] J. Javier Brey, M. J. Ruiz Montero and D. Cubero, Phys. Rev. E, 543664 (1996).

[BrSp96] N. V. Brilliantov, F. Spahn, J. M. Hertzsch and T. Poeschel, Phys. Rev. E, 53, 5382 (1996).

[BBGKY] Bogoliubov, N.N, Problems of Dynamical Theory in Statistical Physics (in Russian). Gostekhizdat, Moscow-Leningrad (1946); M. Born, and H. S. Green: Proc. Roy. Soc. Lond. 188 10, (1946); J. G.Kirkwood, , J. Chem. Phys. 14, 180 (1946); J. Yvon, La theorie statistique des fluids et l' equation d' eta, Actualites Scientifiques et Industrielles, No. 203, 1935.

[Ce95] C. Cercignani in "Fisica matematica et ingegneria delle strutture: rapporti e compatibilita", ed. G. Ferrarese, Pitagora Editrice, p.119, Bologna (1995). p.119.

[ChCo60] S. Chapman and T. G. Cowling, The Mathematical Theory of Nonuniform Gases, Cambridge University Press, London (1960).

[ChGr99] The University of Chicago Granular Physics Group, http://mrsec.uchicago.edu/granular/, (1999).

[Ci86] B. Cichocki, Z. Phys. B 66, 537 (1987).

[Deba97] P. Deltour and J.-L. Barrat, J. Phys. I France 7, 137 (1997).

[ErDo69] M. H. Ernst, J. R. Dorfmann, W. R. Hoegy, and J. M. J. van Leeuwen, Physica 45, 127 (1969).

[ErWo81] J. J. Erpenbeck and W. W. Wood, J. Stat. Phys. 24, 455 (1981).

[EsPo97] S. E. Esipov and T. Pöschel, J. Stat. Phys. 86, 1385 (1997).

[FoLo94] S. F. Foerster, M. Y. Louge, H. Chang and K. Allia, Phys. Fluids 6, 1108 (1994).

[FrMa83] D. Frenkel and J. F. Maguire, Mol. Phys. 49, 503 (1983).

[FuMa75] P. M. Furtado, G. F. Mazenko and S. Yip, Phys. Rev. A12, 1653 (1975).

[GeZi99] F. Gerl and A. Zippelius, Phys. Rev. E, 59, 2361 (1999).

[Ge99] P. G. de Gennes, Rev. Mod. Phys., 71, 374 (1999).

[Gi96] G. Giese, Dissertation, Dynamik granularer Teilchen mit internen Freiheitsgraden, Göttingen (1996). 
[GiZi96] G. Giese and A. Zippelius, Phys. Rev. E 54, 4828 (1996).

[GoSh95] A. Goldshtein and M. Shapiro, J. Fluid Mech.282, 75 (1995).

[GoZa93] I. Goldhirsch and G. Zanetti, Phys. Rev. Lett. 70, 1619 (1993).

[GoZa93b] I. Goldhirsch, M. L. Tan, and G. Zanetti, J. Sci. Comp. 8, 1619 (1993).

[Gr58] H. Grad Principles of the kinetic theory of gases in Handbuch der Physik, ed.

S. F. Fluegge, Springer (1958).

[Ha83] P. K. Haff, Journ. of Fluid. Mech., 134401 (1983).

[Ha86] J.-P. Hansen and I. R. McDonald Theory of Simple Liquids, Academic Press (1986).

[He99] O. Herbst, private communication (1999).

[HeHo98] Physics of dry granular media, Vol. 350 of NATO Advanced Study Institute Series E, edited by H. J. Herrmann, J.-P. Hovi, and S. Luding (Kluwer Academic Publishers, Dordrecht, Netherlands, (1998).

[HoSh87] M. A. Hopkins and H. Shen in Micromechanics of Granular Materials, eds. M. Satake and J. T. Jenkins, Amsterdam, Elsevier, (1987).

[HuAs99] M. Huthmann, T. Aspelmeier and A. Zippelius, Phys. Rev. E 60, 654 (1999).

[HuOr99] M. Huthmann, J. A. G. Ozra, R. Brito, (unpublished) (1999).

[HuZi97] M. Huthmann and A. Zippelius, Phys. Rev. E 56, R6275 (1997).

[IcHa95] K. Ichiki and H. Hayakawa, Phys. Rev. E 52656 (1995).

[JaNa96] H. M. Jaeger, S. R. Nagel and R. P. Behringer, Rev. Mod. Phys. 68, 1259 (1996).

[JeRi85] J. T. Jenkins and M. W. Richman, Phys. of Fluids 28, 3485 (1985).

[JeRi85b] J. T. Jenkins and M. W. Richman, Arch. Ration. Mech. Anal. 87, 355 (1985).

[JeSa83] J. T. Jenkins and S. B. Savage, J. Fluid Mech. 130, 187 (1983).

[Ka99] L. P. Kadanoff, Rev. Mod. Phys. 71, 435 (1999).

[Ko97] M. Kofler, Maple: An Introduction and Reference, Addison-Wesley, Harlow (1997). 
[KoLe64] H. H. U. Konijnendijk and J. M. van Leeuwen, Physica 64, 342 (1973).

[Le82] E. Leutheusser, J. Phys. C 15, 2801 (1982).

[LePe69] J. L. Lebowitz, J. K. Percus and J. Sykes, Phys. Rev. 188, 487 (1969).

[Lu91] C. K. K. Lun, J. Fluid Mech. 233, 539 (1991).

[Lu98] S. Luding in [HeHo98], 285 (1998).

[Lub91] B. D. Lubachevsky, J. of Comp. Phys. 94, 255 (1991).

[LuHu98] S. Luding, M. Huthmann, S. McNamara, A. Zippelius, Phys. Rev. E 58, 3416 (1998).

[LuNa98] S. Luding and S. McNamara, J. Gran. Matt. 1, 113 (1998).

[LuSa84] C. K. K. Lun, S. B. Savage, D. J. Jeffrey and N. Chepurniy, J. Fluid Mech.140, 223 (1984).

[LuSa87] C. K. K. Lun and S. B. Savage, J. Appl. Mech. 54, 47 (1987).

[MaBe94] R. Mazighi, B. Bernu, and F. Delyon, Phys. Rev. E 50, 4551 (1994).

[MaOb66] W. Magnus, F. Oberhettinger, and R. P. Soni, Formulas and Theorems for the Special Functions of Mathematical Physics, Springer (1966).

[MuLu97] M. Müller, S. Luding and H. J. Herrmann. Simulations of a vibrated granular media in 2D and 3D. World Scientific, Singapore (1997).

[Na93] S. McNamara, Phys. Fluids A 5, 3056 (1993).

[NaLu98] S. McNamara and S. Luding, Phys. Rev. E 58, 2247 (1998).

[NaYo94] S. McNamara and W. R. Young, Phys. Rev. E 50, R28 (1994).

[NaYo96] S. McNamara and W. Young, Phys. Rev. E 53, 5089 (1996).

[NoBr98] T. P. C. van Noije, R. Brito and M. H. Ernst, Phys. Rev. E 57, R4891 (1998).

[NoEr97] T. P. C. van Noije, M. H. Ernst, R. Brito and J. A. G. Orza, Phys. Rev. Lett. 97, 411 (1997).

[NoEr97b] T. P. C. van Noije, M. H. Ernst, Physica A 251, 266 (1998).

[NoEr98] T. P. C. van Noije, M. H. Ernst, R. Brito, Physica A 251266 (1998).

[NoEr98b] T. P. C. van Noije, M. H. Ernst, Granular Matter, 1, 57 (1998). 
[NoEr99] T. P. C. van Noije and M. H. Ernst, cond-mat/9907012, (1999).

[PrTe94] W. H. Press, S. A. Teukolsky, W. T. Vetterling, and B. P. Flannery, Numerical Recipes in $C$ : the art of scientific computing 2nd. ed, Cambridge, UK, Cambridge University Press (1994).

[Re75] P. Resibois, J. Stat. Phys. 13, 393 (1975).

[ReLe75] P. Resibois and J. L. Lebowitz, J. Stat. Phys. 12, 483 (1975).

[SeGo96] N. Sela, I. Goldhirsch, and S. H. Noskowicz, Phys. Fluids 8, 2337 (1996).

[TaGo98] M.-L. Tan and I. Goldhirsch, Phys. Rev. Lett. 81, 3022 (1998); Comment by J. W. Dufty J. J. Brey, Phys. Rev. Lett. 82 4566, (1999); Reply by M.-L. Tan and I. Goldhirsch, Phys. Rev. Lett. 824567 (1999).

[TaTa95] Y.-H. Taguchi and H. Takayasu, Europhys. Lett. 30, 499 (1995).

[Th63] E. Thiele, J. Chem. Phys. 39, 474 (1963).

[Ve67] L. Verlet, Phys. Rev. 159, 98 (1967).

[VeLe82] L. Verlet and D. Levesque, Mol. Phys. 46, 969 (1982).

[Wa58] L. Waldmann Transporterscheinungen in Gasen von mittlerem Druck in Handbuch der Physik, ed. S. F. Fluegge, Springer (1958).

[Wa88] O. Walton, in Energy and Technology Review, edited by A. J. Poggio, (Lawrence Livermore National Laboratory, Livermore, CA) (1988).

[We63] M. S. Wertheim, Phys. Rev. Lett. 10, 321 (1963); J. Math. Phys. 5, 634 (1964).

[ZuMo96] D. Zubarev, V. Morozov and G. Röpke, Statistical Mechanics of Nonequilibrium Processes, Akademie Verlag, Berlin (1996). 


\section{Danksagung}

Ich danke Frau Professor Annette Zippelius, daß sie mir die Möglichkeit gegeben hat, an einem aktuellen und spannenden Thema zu arbeiten. Besonders dankbar bin ich für den gewährten Freiraum und das Vertrauen, meine Ideen verwirklichen zu können, und für die stets anregenden und fruchtbaren Diskussionen mit ihr, die mich immer wieder von neuem nachdenken ließen. Vor allem aber hat es Spaß gemacht, mit ihr zu arbeiten.

Ich danke Herrn Professor Reiner Kree, ohne seine begeisternden Vorlesungen wäre ich nie in der Villa gelandet. Ich habe viel von seinem umfassenden Wissen und seinen Anregungen profitiert, nicht zuletzt auch von den vielen nicht wissenschaftlichen Gesprächen.

Quiero agradecer también al Profesor Ricardo Brito la oportunidad de trabajar con él y con José Antonio García Orza en la Universidad Complutense de Madrid. Y a los dos, tanto por lo productiva que ha sido esta colaboración, como por el cálido trato humano que recibí en la vida diaria y en ayudarme a conocer y apreciar Espana, donde querría mencionar también a Marisol.

Besonderen Dank gilt Timo Aspelmeier, der mir mit seinen C++ Künsten viel Arbeit erspart und mir viel Zeit geopfert hat, ohne seinen Anteil an den Simulationsprogrammen und seine ständige Hilfsbereitschaft wäre diese Doktorarbeit so nie fertig geworden.

Eine große Hilfe war die ständige Gesprächsbereitschaft und der Spaß am gemeinsamen Nachdenken in der Villa. Viele und vor allem fruchtbare Diskussionen hatte ich mit Kurt Broderix, Peter Müller und Matthias Otto. Dank auch an die Korrektoren und die Korrektorin dieser Arbeit, Timo Aspelmeier, Olaf Herbst, Sylvia Heudecker, Peter Müller und Matthias Otto.

Viel profitiert und gelernt habe ich auch durch die Zusammenarbeit mit Stefan Luding und Sean McNamara.

Nicht zu unterschätzen ist das angenehme Klima in der Villa, das ohne „unsere" Gisela so nicht denkbar wäre. Sie hat viel dazu beigetragen, daß ich immer wieder gerne in die Villa kam. Auch allen anderen und ehemaligen Villabewohnern sei dafür Dank.

Ich danke allen, die mir auf ihre Weise immer wieder Mut zugesprochen haben. Meinen Schwestern Angela und Anita, Désirée, Dietrich, Julia, Marisol, Michael, Ralf, Sven, Tomas, allen anderen und besonders Sylvia.

Ich danke meinen Eltern für ihre ständige Unterstützung und vor allem für das geschenkte Selbstvertrauen in meine Fähigkeiten.

Diese Arbeit wurde gefördert durch ein Stipendium des Landes Niedersachsen gewährt durch die Universität Göttingen und durch die Deutsche Forschungsgemeinschaft im Rahmen des Sonderforschungsbereichs 345. 


\section{List of publications}

1. M. Huthmann, M. Rehkopf, A. Zippelius, and P. M. Goldbart

Universality of gelation: end-linking versus cross-linking and stiff rods versus flexible chains

Phys. Rev. E 54, 3943 (1996).

2. M. Huthmann and A. Zippelius

Dynamics of inelastically colliding rough spheres: relaxation of translational and rotational energy

Phys. Rev. E 56, R6275 (1997).

3. S. Luding, M. Huthmann, S. McNamara, and A. Zippelius

Homogeneous cooling of rough, dissipative particles: Theory and simulations Phys. Rev. E 58, 3416 (1998).

4. M. Huthmann, T. Aspelmeier, and A. Zippelius

Granular cooling of hard needles

Phys. Rev. E 60, 654 (1999).

5. T. Aspelmeier, M. Huthmann and A. Zippelius

Free cooling of particles with rotational degrees of freedom

to be published in Granular Matter, Lecture Notes in Physics, eds. S. Luding and T. Pöschel, Springer (2000).

6. Olaf Herbst, Martin Huthmann and A. Zippelius

Dynamics of inelastically colliding spheres with Coulomb friction: Relaxation of translational and rotational energy

cond-mat/9911306 (1999). 